This item was submitted to Loughborough's Research Repository by the author.

Items in Figshare are protected by copyright, with all rights reserved, unless otherwise indicated.

\title{
Trapping (capture) into resonance and scattering on resonance: summary of results for space plasma systems
}

\author{
PLEASE CITE THE PUBLISHED VERSION
}

https://doi.org/10.1016/j.cnsns.2018.05.004

\section{PUBLISHER}

(C) Elsevier

\section{VERSION}

AM (Accepted Manuscript)

\section{PUBLISHER STATEMENT}

This work is made available according to the conditions of the Creative Commons Attribution-NonCommercialNoDerivatives 4.0 International (CC BY-NC-ND 4.0) licence. Full details of this licence are available at: https://creativecommons.org/licenses/by-nc-nd/4.0/

\section{LICENCE}

CC BY-NC-ND 4.0

\section{REPOSITORY RECORD}

Artemyev, A.V., Anatoly Neishtadt, D.L. Vainchtein, Alexei Vasiliev, I.Y. Vasko, and L.M. Zelenyi. 2019. "Trapping (capture) into Resonance and Scattering on Resonance: Summary of Results for Space Plasma Systems". figshare. https://hdl.handle.net/2134/33227. 


\title{
Trapping (capture) into resonance and scattering on resonance: summary of results for space plasma systems
}

\author{
A.V. Artemyev ${ }^{1,2}$, A.I. Neishtadt ${ }^{1,3}$, D.L. Vainchtein ${ }^{1,4}$, A.A. Vasiliev ${ }^{1}$, I.Y. Vasko ${ }^{1,5}$, L.M. Zelenyi ${ }^{1,6}$ \\ ${ }^{1}$ Space Research Institute, Moscow 117997, Russia \\ ${ }^{2}$ Department of Earth, Planetary, and Space Sciences, University of California, Los Angeles, CA 90095, USA \\ ${ }^{3}$ Department of Mathematical Sciences, Loughborough University, Loughborough LE11 3TU, United Kingdom \\ ${ }^{4}$ Nyheim Plasma Institute, Drexel University, Camden, NJ 08103, USA \\ 5 Space Sciences Laboratory, University of California, Berkeley, CA 94720, USA \\ ${ }^{6}$ Moscow Institute of Physics and Technology, Dolgoprudny, Moscow Region 141700, Russia
}

\begin{abstract}
In the present review we survey space plasma systems where the nonlinear resonant interaction between charged particles and electromagnetic waves plays an important role. We focus on particle acceleration by strong electromagnetic waves. We start with presenting a general description of nonlinear resonant interaction based on the theory of slowfast Hamiltonian systems with resonances. Then we turn to several manifestations of the resonance effects in various space plasma systems. We describe a universal approach for evaluating main characteristics of the resonant particle dynamics: probability of trapping into resonance, energy change due to scattering and trapping. Then we demonstrate how effects of nonlinear resonant trapping and scattering can be combined in a generalized kinetic equation. We also discuss the stability of trapped motion and evolution of particle ensemble in systems with trapping. The main objective of this review is to provide a general approach for characterizing plasma systems with nonlinear resonant interactions.
\end{abstract}

Keywords: wave-particle resonant interaction, charged particle acceleration and transport, nonlinear waves, adiabatic invariant, probability of trapping

\section{Contents}

1 Introduction $\quad 3$

1.1 Collisionless plasma and electromagnetic waves . . . . . . . . . . . . . . . . . 3

1.2 Quasi-linear theory and nonlinear resonant interaction . . . . . . . . . . . . . . . . 3

1.3 Nonlinear resonant interaction: some examples . . . . . . . . . . . . . . . . . . 4

1.4 Space and laboratory plasma systems with resonant wave-particle interactions . . . . . . . . . . 5

1.5 Review objective and structure . . . . . . . . . . . . . . . . . . . . 7

2 Main equations, averaging, and the derivation of the resonance Hamiltonian 7

3 Trapping (capture) into resonance $\quad 13$

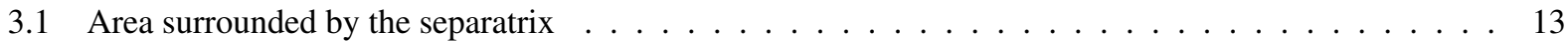

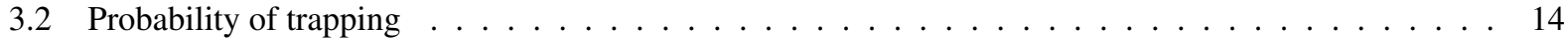

3.3 Non-periodic perturbations . . . . . . . . . . . . . . . . . . . . . 16

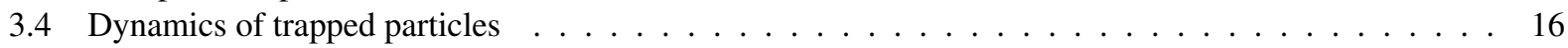

4 Scattering on resonance $\quad 17$

5 Evolution of a particle ensemble $\quad \mathbf{2 0}$

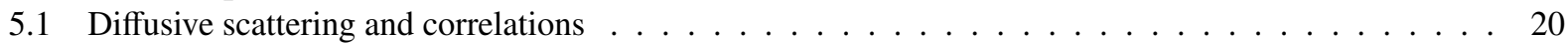

5.1.1 System with a constant frequency . . . . . . . . . . . . . . . . . . 20 
5.1.2 System with a frequency depending on energy . . . . . . . . . . . . . . . 21

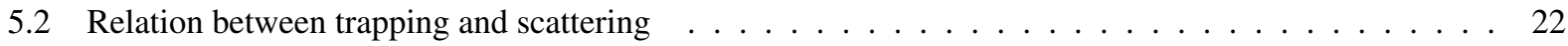

5.3 Kinetic equation for trapped and transient particles $\ldots \ldots \ldots \ldots \ldots \ldots \ldots \ldots \ldots$

6 Particle acceleration in nonrelativistic plasma systems 28

6.1 Electrostatic wave propagating across a weak magnetic field $\ldots \ldots \ldots \ldots \ldots \ldots$

6.2 Front of plasma injection . . . . . . . . . . . . . . . . . . . . . . . 30

6.3 Nonlinear electrostatic localized structures in inhomogeneous magnetic field . . . . . . . . . . 32

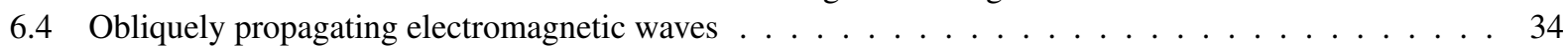

7 Particle acceleration in relativistic plasma systems 37

7.1 Electrostatic wave propagating across a weak magnetic field . . . . . . . . . . . . . . 37

7.2 The Landau resonance in an inhomogeneous magnetic field $\ldots \ldots \ldots \ldots$

7.3 The cyclotron resonance in an inhomogeneous magnetic field $\ldots \ldots \ldots \ldots$. . . . . . . . 42

8 Stability of trapped particle motion $\quad 44$

8.1 Destruction of the trapped motion invariant $\ldots \ldots \ldots \ldots \ldots \ldots \ldots \ldots \ldots$

8.2 Wave-particle resonance in a fluctuating magnetic field $\ldots \ldots \ldots \ldots \ldots \ldots$

9 Discussion and open questions $\quad 51$

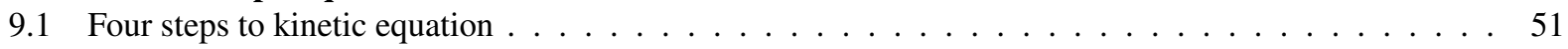

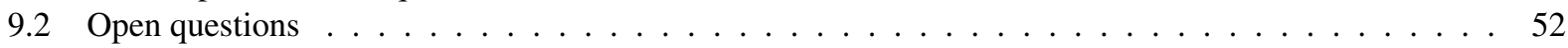

10 Conclusions $\quad 53$

Appendix A Conservation of the adiabatic invariant $I_{\phi}$ and accuracy of integration $\quad 53$

$\begin{array}{lll}\text { Appendix B } & \text { Accuracy of averaging of particle gyrorotation } & 55\end{array}$ 


\begin{tabular}{cl} 
symbol & description \\
\hline$e$ & charge of a particle \\
$m$ & mass of a particle \\
$\mathbf{B}_{0}$ & background magnetic field \\
$\Phi$ & wave scalar potential amplitude \\
$\omega$ & wave frequency; constant in all applications considered in this review \\
$\mathbf{k}, k=|\mathbf{k}|$ & wave number; may depend on spatial coordinates \\
$\varepsilon$ & dimensionless wave amplitude; a small parameter in the systems under consideration \\
$\Omega, \Omega_{0}$ & dimensionless and dimensional frequency of charged particle oscillations in the system with $\varepsilon=0$ \\
$\phi$ & wave phase \\
$F(\phi)$ & profile of a wave field; for most of applications $F=$ sin $\phi$ \\
$u$ & dimensionless function describing the distribution of wave field in space \\
$I$ & momentum conjugate to $\phi$ \\
$I_{r e s}$ & $I$ evaluated at the resonance \\
$P_{\phi}$ & $P_{\phi}=I-I_{\text {res }} ;$ momentum conjugate to $\phi$ \\
$H$ & Hamiltonian of the initial system \\
$\mathcal{F}$ & Hamiltonian after the introduction of conjugate variables $(\phi, I)$ \\
$H_{\phi}$ & a part of Hamiltonian $\mathcal{F}$ describing particle motion in the $\left(\phi, P_{\phi}\right)$ plane \\
$I_{\phi}$ & the area surrounded by closed particle trajectory in the $\left(\phi, P_{\phi}\right)$ plane $($ divided by $2 \pi)$ \\
$S_{r e s}$ & the area surrounded by the separatrix in the $\left(\phi, P_{\phi}\right)$ plane \\
$v_{\phi}, v_{\text {res }}$ & $v_{\phi}=\omega / k$ is phase velocity of $\phi$, and $v_{\text {res }}$ is a dimensionless $v_{\phi}$ \\
$\Psi$ & particle distribution function in the phase space
\end{tabular}

\section{Introduction}

\subsection{Collisionless plasma and electromagnetic waves}

Plasma is a gas of ionized particles. In sufficiently dense plasma, particle collisions control the energy and momentum exchange between particles and transformation of energy from from kinetic to thermal. A characteristic distance between two consecutive collisions, called mean free path, determines spatial scales of the energy dissipation, e.g. shock wave scales and boundary scale between two gas populations [e.g. 1]. In rarefied plasma the mean free path may be very large, even larger than the scale of the entire system. Such plasma systems are effectively collisionless, and in them other kinetic processes control the energy exchange and dissipation. Plasma particles, being charged and affected by electromagnetic fields, can interact with electromagnetic waves. Such waves, generated by one population of particles, can travel in space and interact with another, possible quite distant, particle population. This interaction effectively connects particles which never physically collide with each other, and provides a collisionless momentum exchange. Therefore, wave-particle interaction is a crucial component of any detailed picture of energetic processes in collisionless plasma [e.g., 2, 3].

Electromagnetic waves are periodic (or quasi-periodic) variations of electromagnetic field. For sufficiently smooth fields, change of phase $\phi$ in time equals minus the wave frequency, $\partial \phi / \partial t=-\omega$, and the gradient equals the wavenumber vector, $\nabla \phi=\mathbf{k}$. There are two main regimes of the wave-particle interaction: nonresonant and resonant. If $d \phi / d t=0$ somewhere along a particle trajectory, the wave-particle interaction is resonant. This regime is especially important because the slowed phase represents stationary electromagnetic fields, which act on particles much more effectively than nonresonant fields fluctuating around zero. Systems with the resonant wave-particle interaction can be roughly further divided into two groups depending on character of this interaction.

\subsection{Quasi-linear theory and nonlinear resonant interaction}

In the first group are the systems where the wave-particle resonant interaction can be considered as a stochastic process. Such an interaction lasts a short time and its characteristics (e.g., particle shift in phase space due to the wave action) are randomly distributed in the phase space of the initial particle position and velocity. Due to the shortness 
of resonant interaction, the particle position change during a given interaction is usually small. This allows us to consider the wave-particle interaction in a framework of the perturbation theory. When averaged over unperturbed particle trajectory, oscillating wave field causes a random change of the particle characteristics. These changes have zero mean and their variance is responsible for the diffusion in the phase space. Weakness of wave-particle stochastic interaction and the random nature of this interaction provide a foundation for application of the classical Fokker-Plank equation for description of charged particle evolution [e.g., 4]. Different plasma systems provide different origins of stochastization of charged particle motion, and thus require individual approaches for derivation of coefficients (diffusion coefficients) of the Fokker-Plank equation.

The stochasticity of charged particle resonance with an electromagnetic field is caused by a broadness of wave spectrum: the duration of the wave-particle resonant interaction is mediated by nonresonant waves with frequencies near the resonant one. This effect is a foundation for the quasi-linear theory of wave-particle resonant interaction $[5,6,7,8,9]$. However, even in systems with a narrow wave spectrum (or even with a single coherent wave), there may be an effective stochasticity. One the natural source of such a stochasticity is an inhomogeneity of the background magnetic field/plasma density, [see discussion in 10,11, 12] as gradients of system's characteristics destroy the long-term correlations between the wave and particles [see, e.g., example in 13, 14]. Another source of particle stochasticity is an external nonresonant noise (random fluctuations of the background system parameters), which can also significantly limit the duration of the resonant wave-particle interaction [e.g., 15, 16]. Finally, the internal instability of waves may also result in the wave spectrum spreading and resonance destruction [17, 18]. All these processes justify the applicability of the Fokker-Plank equation (or the quasi-linear theory) for many, but not all, space plasma systems where the wave-particle interactions are crucial for energy transformation and dissipation [e.g., $19,20,21]$. The main property of this, first, group of systems is the weakness of wave-particle interaction allowing using unperturbed trajectories for calculation the cumulative wave effect on particles.

The second group consists of various systems where wave field is sufficiently strong to change particle dynamics and wave effect should be included for calculation of the resonant particle trajectories. Thus, the perturbation theory is no more applicable for investigation of the wave-particle resonant interaction, and this interaction is called nonlinear. In this interaction changes of the resonant particle characteristics due to interaction with waves may have finite (nonzero) mean. Therefore, the resonant wave-particle interaction in systems of the second group may be not diffusive. Such changes essentially depend on the initial particle positions in the phase space. As we usually do not have a detailed information about particle initial distributions with the infinite high accuracy, and thus cannot predict the effect of the nonlinear wave-particle interaction for each particle, we average this effect for large particle ensemble. Such averaging leads to a kinetic equation for particle distribution function, but this equation may differ from the diffusion Fokker-Plank equation because it takes into account significantly nondiffusive effects.

Acceleration/deceleration of particles is closely related to amplification/damping of waves due to the conservation of energy. In many systems the wave energy does not represent a significant energy storage and waves rather play a role of mediator of energy exchange between different particle populations $[22,23]$. Thus, an accurate modeling of wave evolution requires calculation of inputs of different particle populations [24, 25, 26, 27, 28, 29]. Moreover, wave dynamics can be even more complex due to the contribution of trapped particles to the wave dispersion [e.g. 22]. A sufficiently large population of trapped particles may change wave frequency, making it dependent on wave amplitude [e.g., 30, 31, 32, 29, 33]. Such a complicated dynamics of waves requires comprehensive models of wave generation/amplification, but simultaneously significantly simplifies the description of resonant acceleration/deceleration of small particle populations. Indeed, feedback of these acceleration/deceleration to waves can be omitted in the leading approximation, because waves do not provide their own energy to particle acceleration. Thus, consideration of the nonlinear charged particle resonances is often limited to the case of prescribed wave amplitudes and frequencies.

\subsection{Nonlinear resonant interaction: some examples}

Let us start our discussion of nonlinear resonant interactions by introducing three examples that cover a wide range of the resonant effects in plasma systems.

For the nonlinear resonant interaction to occur, the wave electromagnetic fields, $\mathbf{B}_{w}$ and $\mathbf{E}_{w}$, should be sufficiently strong. Roughly speaking, the magnitude of the wave Lorentz force $e c^{-1} \mathbf{v} \times \mathbf{B}_{w}+e \mathbf{E}_{w}$, where $\mathbf{v}$ and $e$ are particle velocity and charge, and $c$ the speed of light, should exceed the magnitude background field Lorentz force $e c^{-1} \mathbf{v} \times \mathbf{B}_{0}$. When the projection of the sum of these two forces on the direction of wave phase changing is zero, resonant particles 


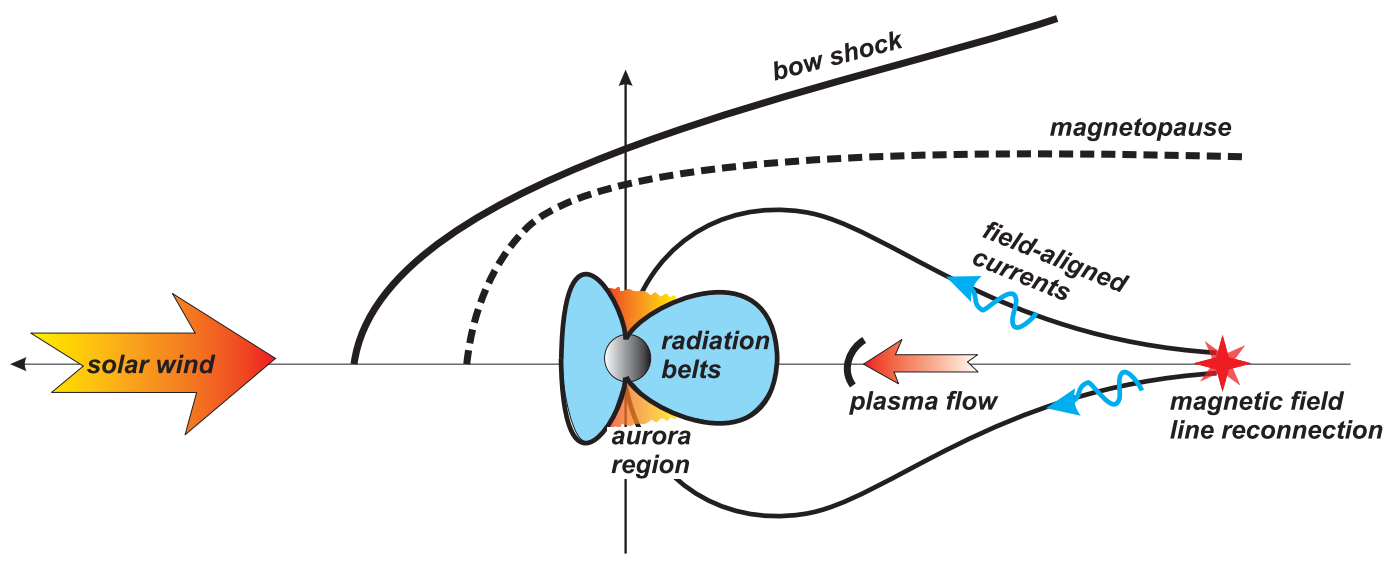

Figure 1: Schematic view of the near-Earth plasma systems.

can spend a long time in the resonance. This effect is called particle trapping or capture into resonance, and it is a unique feature of the nonlinear resonant interaction.

The simplest example of a nonlinear resonance is an electrostatic wave $\mathbf{B}_{w}=0$ propagating in the system without a background field: $\mathbf{B}_{0}=0$. In this case, $\dot{\phi}=(\nabla \phi) \mathbf{v}-\omega=\mathbf{k v}-\omega$. The corresponding resonant condition, $\mathbf{k v}=\omega$, is called the Cherenkov resonant condition: the projection of a particle velocity $\mathbf{v}$ on the direction of the wavevector $\mathbf{k}$ is equal to the wave phase velocity $v_{\phi}=\omega / k$. Particles trapped into the Cherenkov resonance propagate with the electrostatic waves $[34,35]$. If waves propagate in an inhomogeneous plasma, the wave dispersion relation and the conservation of wave frequency [see, e.g., 36] determine the evolution of $v_{\phi}$ and thus the change of the particle velocity $\mathbf{v}=(\mathbf{k} / k) v_{\phi}$. This results in an acceleration/deceleration of trapped particles [37, 38, 39, 40].

A weak background magnetic field $\mathbf{B}_{\mathbf{0}}$ alters the resonant interaction with electrostatic waves only if the wave propagates across the field; for field-aligned waves the resonant interaction is not disturbed by the background Lorenz force. For transversely propagating waves, trapping into the Cherenkov resonance requires $\mathbf{E}_{w}>c^{-1} \mathbf{v} \times \mathbf{B}_{0}$ [41, 42]. Trapped particles move with the wave while experiencing acceleration in the direction transverse to the background field and wave propagation direction: there is an effective force $c^{-1} \mathbf{v}_{\phi} \times \mathbf{B}_{0}$ acting on particle. Such an acceleration is called the surfatron acceleration $[43,44]$.

In systems with a strong background magnetic field, the particle Larmor rotation cannot be significantly disturbed by wave field, but the particle trapping is still possible. In these systems, the particle Larmor rotation has the frequency $\Omega=e\left|\mathbf{B}_{0}\right| / m c$ where $e$ and $m$ are particle charge and mass, and the wave may be in resonance with one of the harmonics: $\omega-k_{\|} v_{\|}=n \Omega$. The subscript $\|$ denotes the projection on direction of the background magnetic field: $\left(v_{\|}=\mathbf{v} \cdot \mathbf{B}_{0} /\left|\mathbf{B}_{0}\right|\right)$, and $n=0, \pm 1, \pm 2, \ldots$ is an integer number. A particular case with $n=0$ is often called the Landau resonance. To trap particles into such a resonance, the wave field should be able to compensate the action of the force, called the mirror force and proportional to the gradient of $\nabla_{\|}\left|\mathbf{B}_{0}\right|$. Particles trapped into resonance move with the resonant velocity $v_{\|}=(\omega-n \Omega) / k_{\|}$and effectively accelerate in the inhomogeneous $\left(\nabla_{\|} \mathbf{B}_{0} \neq 0\right)$ field $[45,46,47]$.

\subsection{Space and laboratory plasma systems with resonant wave-particle interactions}

Nonlinear wave-particle resonances play an important role in many near-Earth space plasma systems, schematically shown in Fig. 1. Early in-situ spacecraft observations demonstrated that beyond the ionosphere the near-Earth space was filled by plasma originating from the ionosphere (ionosphere outflow) and from the solar wind. The mean free path for such plasma exceeds the distance between Earth and Sun $\left(>10^{8} \mathrm{~km}\right)$, whereas spatial scales of many plasma systems discovered in near-Earth space do not approach $10^{2}-10^{4} \mathrm{~km}$. Thus, only the wave-particle interactions can control energy transformation and dissipation in these collisionless systems. Below we describe several examples of such space plasma systems and discuss the importance of wave-particle interaction.

The collisionless solar wind propagating from the Sun does not evolve as an expanding gas, but exhibits a complicated internal dynamics where strong Alfven, magnetosonic, and electrostatic waves play a critical role in energy exchange between different particle populations [48]. The solar wind temperature decreases with the distance from 
the Sun significantly slower than it should according to the adiabatic theory of expanding gas. This slowing of cooling is believed to be caused by the interaction of charged particles with Alfven and whistler wave turbulence dominating in the solar wind magnetic field spectra [e.g., 49, 50]. Waves can also be responsible for the formation and dynamics of fine structures. Multiple magnetic field discontinuities observed in the solar wind [e.g., 51, 52, 53, and references therein] are formed due to Alfven waves nonlinear evolution driven by the Landau resonance with ions [e.g., 54, 55]. Another example is the electron resonance with strong Langmuir (electrostatic) waves propagating though the inhomogeneous solar wind plasma $[56,57]$. This nonlinear interaction is responsible for wave evolution and electron acceleration $[58,59]$.

Upon reaching the Earth bow shock, the solar wind transforms from a supersonic to subsonic flow. This transformation and corresponding plasma thermalization are accompanied by the formation of a broad spectrum of electromagnetic waves. Strong electrostatic waves at the bow shock [e.g., 60] trap electrons into the Landau resonance and can significantly change their distributions in the phase space. Electromagnetic whistler and ion cyclotron waves $[61,62]$ trap electrons and ions into the cyclotron resonances [e.g., 63, 64], and the corresponding particle acceleration can describe fluxes of high-energy particles observed at the bow shock [e.g., 65, 66, 67]. Moreover, the formation and evolution of the Earth bow shock is significantly affected by the nonlinear resonant interaction of ions with electromagnetic waves emitting by the shock [see, e.g., review 68, and references therein].

Shocked solar wind plasma fills the magnetosheath, which is the region between the bow shock and the magnetopause. Magnetopause is a thin boundary separating the solar wind from the magnetospheric plasma. One of the most important questions of the Earth magnetosphere physics is how the magnetosheath plasma penetrates across the magnetopause [see review 69]. In absence of particle collisions and/or magnetopause destruction, the only working mechanism of plasma transport to the magnetosphere is by the wave-particle resonant interaction [e.g., 70]. Abundance of cyclotron [e.g., 71, 72] and Alfven [e.g., 73] waves at the magnetopause supports the scenario of the resonant particle scattering and/or trapping causing the diffusion across the magnetopause [74, 75].

Plasma may penetrate into the magnetosphere tail through the magnetopause. Penetrated plasma generates currents flowing in the magnetotail current sheet. Such currents are a significant free energy source, which makes the magnetotail plasma distribution highly unstable. The release of this energy is controlled by reconnections of magnetic field lines. This process transforms magnetic field energy into the charged particle acceleration and heating [e.g., $76,77,78$ ] and generates various electromagnetic waves [79]. These waves transport some portion of released energy from the reconnection region into the inner magnetosphere [e.g., 80] and the aurora region [e.g., 81, 82].

Energy and plasma transported into the inner magnetosphere from the magnetotail play a crucial role in plasma dynamics in the near-Earth environment. Hot ions injected into the inner magnetosphere form the ring current region and generate strong electric currents changing the near-Earth magnetic field [e.g., 20, 83]. Ring currents are essentially controlled by the ion resonant trapping and scattering by electromagnetic ion cyclotron waves [e.g., 84, and references therein]. In contrast to ions, injected electrons do not contribute significantly to local electric currents, but generate strong electromagnetic (whistler) waves, that determine the dynamics of the radiation belts [85, 86, 87] and accelerate electrons to relativistic energies [22,88].

Released energy, transported into aurora region, fuels aurora electron acceleration and precipitation into the Earth ionosphere. In absence of collisions, this energy absorption is supported by the wave-particle interaction. Currents and magnetohydrodynamic waves, transporting energy to the aurora region, generate strong kinetic Alfven waves carrying electric fields parallel to the background magnetic field [e.g., 89, 75]. These waves resonate with cold electrons and trap them into the Landau resonance, which results in an effective electron acceleration [90, 91, 92].

Investigation of space plasma stimulated experimental laboratory simulations of wave generation and resonant charged particle dynamics, which contributed significantly to the understanding of the nonlinear wave-particle resonances. Unlike the spacecraft observations, in laboratory modelling the wave characteristics could be precisely controlled. Among important laboratory simulations are the investigations of the wave generation by plasma beam [e.g., 93, 94], the nonlinear wave dynamics [e.g., 95], and energy exchange between particle populations through wave generation and absorption [e.g., 96].

Nonlinear wave-particle resonances observed in space plasma have direct analogies in many laboratory experiments. Strongly nonlinear electron acceleration by intense Langmuir waves in inertial confinement fusion experiments [97] can significantly influence plasma conditions [98]. Appearance of accelerated particle populations in the Free-Electron Laser experiments with Variable Parameter Wigglers [99, 100] is described by essentially the same equations as trapping into the cyclotron resonance with electromagnetic waves. Generation or injection of strong 
waves into mirror machines result in a strong wave-particle interaction and excitation of sideband waves induced by particle trapping [e.g., 101, 102]. Electron surface acceleration in the ultraintense laser pulse experiments represents a classical example of electron trapping into the Cherenkov resonance [103].

\subsection{Review objective and structure}

The nonlinear resonance interactions between particles and waves are well described for individual particle trajectories. Using the Hamiltonian mechanics for systems with resonances and introducing a hierarchy of time-scales of particle motion, one can describe particle trapping and scattering for many realistic plasma systems. However, the impact of individual resonant interactions on shaping of particle distribution function as a whole remains not understood. Beyond the diffusive approximation of a weak wave-particle interaction, there is no universal kinetic equation describing a cumulative effect of many nonlinear resonant interactions on the long-term evolution of particle distribution functions. To develop such a new kinetic approach, principal characteristics of the nonlinear resonance processes (the relative number of resonant particles trapped into resonance, average gain of energy in trapping, amplitude of the nonlinear scattering in velocity space) should be evaluated and combined into a generalized kinetic (Fokker-Plank) equation. The main objective of this review is to present a standardized algorithm realising this approach.

The review consists of ten sections. Past the Introduction, in the first part of the review (Sects. 2-5), we present a general theory of scattering on resonance and trapping into resonance (also called capture into resonance) in terms of the theory of adiabatic invariants [104]. We start with main equations and use simple models of physical systems to illustrate the separation of time scales and the introduction of resonance Hamiltonian (Sect. 2). Then we describe the details of trapping into resonance and illustrate the calculation of probability of trapping (Sect. 3). Section 4 describes the nonlinear scattering on resonance. Then we discuss effects of trapping and scattering on dynamics of a charged particle ensemble (Sect. 5), in particular, stochastization of wave-particle resonances (Sect. 5.1), relations between trapping and scattering characteristics (Sect. 5.2), and pathways to a generalized kinetic equation (Sect. 5.3).

In the second part of the review (Sects. 6-7), we consider different concrete plasma systems where effects of trapping play an important role. These systems differ by the configuration of the background electromagnetic field and/or properties of the waves. Different configurations result in different types of resonances: the Cherenkov resonance for systems with a weak background magnetic field, the Landau resonance for particles interacting with electrostatic waves propagating along a strong background magnetic field, and the cyclotron resonance for particles interacting with electromagnetic waves propagating along a strong background magnetic field. We separate all systems into nonrelativistic and relativistic categories. The principal difference between these two groups is that the period of particle oscillations in a background magnetic field depends on particle energy in relativistic systems, whereas in nonrelativistic systems the period of particle gyrorotation does not depend on energy. Each subsection of (Sects. 6-7) discusses one particular physical system. Our objective is to demonstrate that for different electromagnetic fields trapping can be described by the same universal approach described in (Sects. 3-5). The important question of the nonlinear wave-particle resonance is how particle trapping is table relative to the external electromagnetic field fluctuations. The most dangerous resonant sideband fluctuations can easily destroy the nonlinear resonances [155, 17, 18]. However, even in absence of resonant fluctuations, the nonresonant fluctuation can results in particle detraping. This effect is considered in Sect. 8, where we discuss the destruction of the nonlinear resonant interaction. In the last two section, we present open questions, discussion, and conclusions.

\section{Main equations, averaging, and the derivation of the resonance Hamiltonian}

Charged particle motion in electromagnetic fields is described by a 3D Hamiltonian possibly depending on time. However, the symmetry of magnetic field configuration can reduce the number of degrees of freedom. Almost all plasma systems considered in this review can be described by Hamiltonian systems with $1 \frac{1}{2}$ degrees of freedom (two conjugate variables $\left(x, p_{x}\right)$ and time $\left.t\right)$. In dimensionless units, the Hamiltonian of such a system can be written as

$$
H=G\left(x, p_{x}\right)+\varepsilon u\left(x, p_{x}\right) F(\phi)
$$


where $G\left(x, p_{x}\right)$ is a Hamiltonian of an unperturbed motion, $\varepsilon u\left(x, p_{x}\right)$ is an effective amplitude of perturbations $(|\varepsilon| \ll 1$ and $|u| \sim 1$ for all $\left(x, p_{x}\right)$ ), and $F(\phi)$ describes a profile of perturbation as a function of phase $\phi$ :

$$
\phi=\phi_{0}+\int^{x} k(\tilde{x}) d \tilde{x}-\omega t
$$

with $\phi_{0}$ being an initial phase. Frequency of phase $\omega$, and wave number $k(\tilde{x})$ describe evolution of phase in space and time. Both $\omega$ and $k$ are assumed to be large enough (whereas their ratio is about 1) to provide a much faster rate of change of phase $\phi$ compared with variations of variables $\left(x, p_{x}\right)$.

In what follows, all general equations are accompanied by examples obtained for three Hamiltonian systems. The first system describes a linear oscillator in the $\left(x, p_{x}\right)$ plane (see Subsect. 6.1 with examples of the corresponding physical system):

$$
H=\frac{1}{2} p_{x}^{2}+\frac{1}{2} \Omega^{2} x^{2}+\varepsilon u \sin \phi
$$

with $k=$ const, $u=1$, and $\Omega$ is an dimensionless frequency of oscillations in the $\left(x, p_{x}\right)$ plane.

The second system describes nonlinear oscillator with

$$
H=\sqrt{1+p_{x}^{2}+\Omega^{2} x^{2}}+\varepsilon u \sin \phi
$$

and $k=$ const, $u=1$ (see Subsect. 7.1 with examples of the corresponding physical system).

The third system describes a linear oscillator and perturbations with an inhomogeneous amplitude

$$
H=\frac{1}{2} p_{x}^{2}+\frac{1}{2} \Omega^{2} x^{2}+\varepsilon x \sin \phi
$$

with $k=$ const, $u=x$ (see Subsect. 6.2 with examples of the corresponding physical system).

In system (1), the time dependence given by Eq. (2) can be conventionally changed to a new pair of conjugate variables. This change of variables can be performed in two steps. The first step consists of the introduction of a new coordinate $\psi=\omega t$ and the conjugate momentum $p_{\psi}$. To keep the Hamiltonian form of equations, Hamiltonian (1) should be rewritten as:

$$
H_{\psi}=G\left(x, p_{x}\right)+\varepsilon u\left(x, p_{x}\right) F(\phi)+\omega p_{\psi}, \quad \phi=\phi_{0}+\int^{x} k(\tilde{x}) d \tilde{x}-\psi
$$

This transformation is not canonical because number of variables increased: instead of $1 \frac{1}{2}$ degrees of freedom in system (1) we obtained system (6) with 2 degrees of freedom. However, the Hamiltonian equations coincide for both systems. Further in the text, we will skip this step and start with the second step that corresponds to introduction of new variables $\left(X, P_{x}\right),(\phi, I)$ with the generation function

$$
R=I\left(\phi_{0}+\int^{x} k(\tilde{x}) d \tilde{x}-\psi\right)+P_{x} x
$$

Equation (7) gives the following relations between the new and old variables:

$$
p_{\psi}=\frac{\partial R}{\partial \psi}=-I, \quad p_{x}=\frac{\partial R}{\partial x}=P_{x}+I k, \quad X=x
$$

The new Hamiltonian takes the form:

$$
\mathcal{F}=-\omega I+G\left(x, P_{x}+I k\right)+\varepsilon u\left(x, P_{x}+I k\right) F(\phi)
$$

where $k=k(x)$ and we kept the notation $x$ for $X$. Hamiltonian (9) does not depend on time and thus the energy $h=\mathcal{F}$ is conserved. In terms of Hamiltonian mechanics, $I$ is a momentum in Hamiltonian (9). Comparing Eq. (9) 


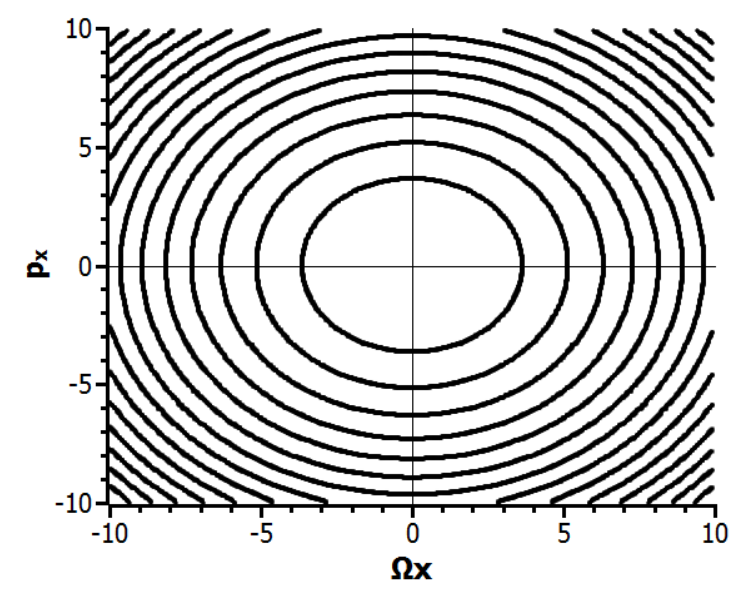

Figure 2: Phase portraits of systems (3) and (4).

and Eq. (1), and taking into account the conservation of $h$, a change of energy $H$ in unperturbed system (with $F=0$ ) equals to change of $\omega I$. In other words, the changes of energy $H$ of the initial system follow the changes in $\omega I$.

Hamiltonian equations for Hamiltonian (9) can be written as:

$$
\begin{aligned}
\dot{x} & =\frac{\partial \mathcal{F}}{\partial P_{x}}=\frac{\partial G}{\partial P_{x}}+\varepsilon \frac{\partial u}{\partial P_{x}} F \\
\dot{P}_{x} & =-\frac{\partial \mathcal{F}}{\partial x}=-\frac{\partial G}{\partial x}-\varepsilon \frac{\partial u}{\partial x} F \\
\dot{\phi} & =\frac{\partial \mathcal{F}}{\partial I}=k \frac{\partial G}{\partial P_{x}}-\omega+\varepsilon k \frac{\partial u}{\partial P_{x}} F \\
\dot{I} & =-\frac{\partial \mathcal{F}}{\partial \phi}=-\varepsilon u \frac{d F}{d \phi}
\end{aligned}
$$

These equations show that the rate of change of variables $\left(x, P_{x}\right)$ is $\sim 1$ (it does not depend on small parameter $\varepsilon$ ), the rate of change for $k I$ is about $k \varepsilon$ (a product of a small parameter $\varepsilon$ and a large parameter $k$ ), and the rate of change of $\phi$ is of the order of a large parameter $k$. We assume that $\varepsilon k \sim 1$. In this case, Hamiltonian system (10) contains a fast phase $\phi$ and slow variables $\left(x, P_{x}\right)$ and $I$. Averaging over the fast phase $\phi$ gives the following equations of motion:

$$
\dot{x}=\frac{\partial G}{\partial P_{x}}+\varepsilon \frac{\partial u}{\partial P_{x}}\langle F\rangle, \quad \dot{P}_{x}=-\frac{\partial G}{\partial x}-\varepsilon \frac{\partial u}{\partial x}\langle F\rangle, \quad \dot{I}=0
$$

where

$$
\langle F\rangle=\lim _{\phi_{l} \rightarrow \infty} \frac{1}{2 \phi_{l}} \int_{-\phi_{l}}^{\phi_{l}} F(\phi) d \phi
$$

For periodic perturbations (e.g., $F \sim \sin \phi$ ) we have $\langle F\rangle=0$. Moreover, even perturbations with nonzero values of $\langle F\rangle$ affect the Hamiltonian equations only slightly, $\sim \varepsilon$. Therefore, $I$ can be considered as an adiabatic invariant: it is conserved in the system averaged over $\phi$ and changes only if separation of time-scales is (the assumption that the phase $\phi$ is fast) is violated. In other words, the conservation of $I$ breaks down when $\dot{\phi} \sim 0$ ).

Consider unperturbed system (1) with Hamiltonian $G\left(x, P_{x}\right)$. We restrict our consideration to the class of systems where the motion in the $\left(x, P_{x}\right)$ plane is finite: phase points move along closed trajectories (see and example of characteristic phase portraits in Fig. 2). The perturbation $\varepsilon u F$ barely deforms trajectories in the $\left(x, P_{x}\right)$ plane everywhere where the averaging over fast phase $\phi$ can be performed. This averaging breaks down in the vicinity of the resonance 
$\dot{\phi}=0$, where the rate of change of the phase drops to zero. In $3 \mathrm{D}$ space $\left(x, P_{x}, I\right)$, the resonant condition is satisfied on the surface $I_{\text {res }}=I_{\text {res }}\left(x, P_{x}\right)$, which can be determined by setting $\dot{\phi}=0$ in system (10):

$$
k \frac{\partial G}{\partial P_{x}}-\omega=0
$$

with $I_{\text {res }}$ being defined in the zeroth-order approximation (i.e., for $\varepsilon=0$ ). For systems (3) and (5), Eq. (13) becomes

$$
k\left(P_{x}+k I_{r e s}\right)-\omega=0
$$

The corresponding solution is

$$
I_{\text {res }}=\frac{1}{k}\left(v_{\text {res }}-P_{x}\right)
$$

where $v_{\text {res }}=\omega / k$. For system (4), Eq. (13) is

$$
k I_{r e s}=v_{\text {res }} \sqrt{1+\left(P_{x}+k I_{r e s}\right)^{2}+\Omega^{2} x^{2}}-P_{x}
$$

Solution of Eq. (16) is

$$
I_{\text {res }}=\frac{1}{k}\left(\frac{v_{r e s} \sqrt{1+\Omega^{2} x^{2}}}{\sqrt{1-v_{r e s}^{2}}}-P_{x}\right)
$$

Combination of equation $I_{r e s}=I_{r e s}(x, P)$ and unperturbed Hamiltonian (9) defines resonant curves $P_{r e s}=P_{r e s}(x)$ in the $\left(x, P_{x}\right)$ plane or $p_{x, r e s}=p_{x, r e s}(x)$ in the $\left(x, p_{x}\right)$ plane:

$$
h=-\omega I_{\text {res }}+G\left(x, P_{\text {res }}+k I_{\text {res }}\right)
$$

where $h$ is a conserved energy for Hamiltonian (9). For functions (3) and (5) these curves are

$$
P_{\text {res }}=\frac{1}{v_{\text {res }}}\left(h+\frac{1}{2} v_{\text {res }}^{2}-\frac{1}{2} \Omega^{2} x^{2}\right), \quad p_{x, \text { res }}=v_{\text {res }}
$$

while for system (4) they are

$$
P_{\text {res }}=\frac{1}{v_{\text {res }}}\left(h-\sqrt{1-v_{\text {res }}^{2}} \sqrt{1+\Omega^{2} x^{2}}\right), \quad p_{x, \text { res }}=\frac{v_{\text {res }}}{\sqrt{1-v_{\text {res }}^{2}}} \sqrt{1+\Omega^{2} x^{2}}
$$

Figure 3 shows unperturbed trajectories in the $\left(x, p_{x}\right)$ plane and the corresponding resonant curves (19) and (20). Closed unperturbed trajectories are crossed by the resonant curve $p_{x}=p_{x, \text { res }}$ twice. Thus there are two resonance crossings for each period of the unperturbed motion.

Consider a vicinity of the resonance and expand Hamiltonian (9) around $I-I_{\text {res }}=0$ :

$$
\mathcal{F}=-\omega I_{\text {res }}+G_{\text {res }}+\varepsilon u F(\phi)+\left.\frac{1}{2} \frac{\partial^{2} G}{\partial I^{2}}\right|_{I=I_{\text {res }}}\left(I-I_{\text {res }}\right)^{2}
$$

where $G_{r e s}$ is evaluated at $I=I_{\text {res }}$. We introduce new variable $P_{\phi}=\left(I-I_{\text {res }}\right)$ with the generating function

$$
R=\left(P_{\phi}+I_{r e s}\right) \phi+\bar{P}_{x} x
$$

where new conjugate variables $\left(\bar{P}_{x}, \bar{x}\right),\left(P_{\phi}, \bar{\phi}\right)$ are

$$
\begin{aligned}
I & =\frac{\partial R}{\partial \phi}=P_{\phi}+I_{\text {res }} \\
P_{x} & =\frac{\partial R}{\partial x}=\bar{P}_{x}+\frac{\partial I_{\text {res }}}{\partial x} \phi \\
\bar{\phi} & =\phi, \quad \bar{x}=x-\frac{\partial I_{\text {res }}}{\partial \bar{P}_{x}} \phi
\end{aligned}
$$



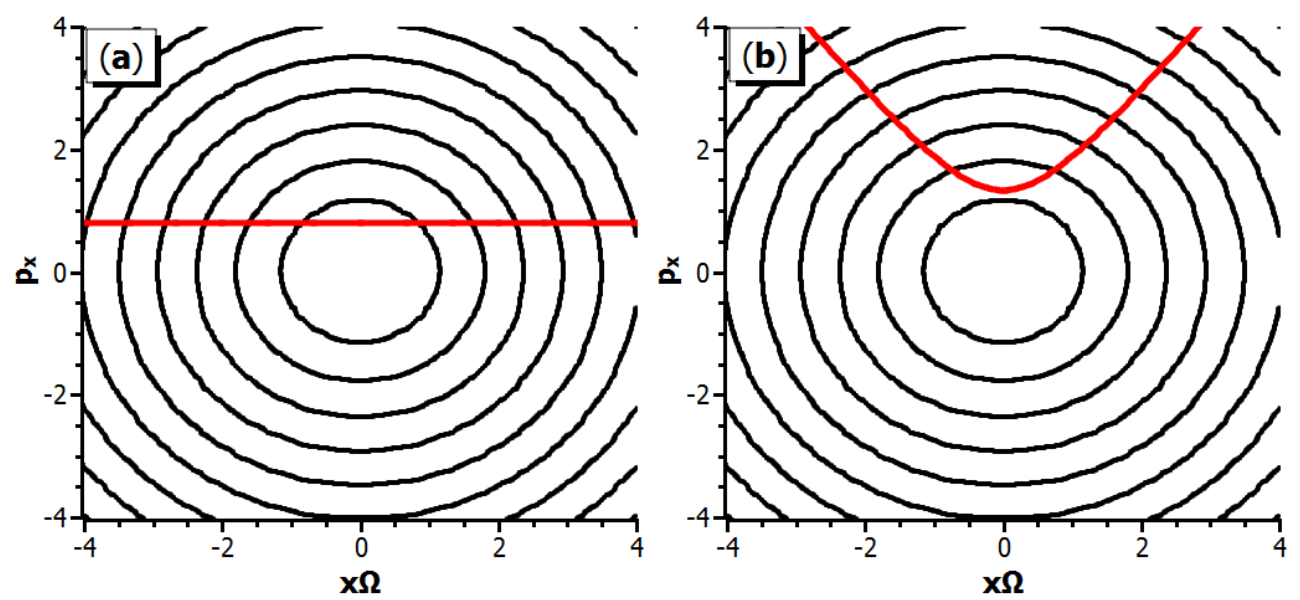

Figure 3: Phase portraits of systems (a)(3) and (b)(4). Red curves are the resonances $p_{x}=v_{r e s}(\mathrm{a}), p_{x}=P_{r e s}+k I_{r e s}(\mathrm{~b})$ defined by Eqs. (20), (17).

New Hamiltonian takes the form

$$
\overline{\mathcal{F}}=-\omega I_{\text {res }}+G_{\text {res }}\left(\bar{x}-\frac{\partial I_{\text {res }}}{\partial P_{x}} \bar{\phi}, \bar{p}_{x}+\frac{\partial I_{\text {res }}}{\partial x} \bar{\phi}\right)+\frac{1}{2} g P_{\phi}^{2}+\varepsilon u F(\bar{\phi})
$$

where

$$
g=\left.\frac{\partial^{2} G}{\partial I^{2}}\right|_{I=I_{\text {res }}}
$$

In Eq. (24), function $G_{r e s}$ depends on $\bar{x}-\left(\partial I_{\text {res }} / \partial P_{x}\right) \bar{\phi}$ and $\bar{p}_{x}-\left(\partial I_{\text {res }} / \partial x\right) \bar{\phi}$. Equation (8) shows that $\partial I_{\text {res }} / \partial P_{x} \sim 1 / k$, $\partial I_{\text {res }} / \partial x \sim 1 / k$, therefore these terms can be considered as perturbations for $\bar{x}, \bar{p}_{x}$ (see also Eqs. $(15,17)$ ). Thus $G_{r e s}$ can be expanded as

$$
\begin{aligned}
G_{r e s}\left(\bar{x}-\frac{\partial I_{r e s}}{\partial P_{x}} \bar{\phi}, \bar{p}_{x}+\frac{\partial I_{\text {res }}}{\partial x} \bar{\phi}\right) & \approx G_{r e s}\left(\bar{x}, \bar{p}_{x}\right)-\frac{\partial I_{\text {res }}}{\partial P_{x}} \frac{\partial G_{r e s}}{\partial \bar{x}} \bar{\phi}+\frac{\partial I_{r e s}}{\partial x} \frac{\partial G_{r e s}}{\partial \bar{p}_{x}} \bar{\phi} \\
& =G_{r e s}\left(\bar{x}, \bar{p}_{x}\right)+\left\{I_{r e s}, G_{r e s}\right\} \bar{\phi}
\end{aligned}
$$

Substituting Eq. (26) into Eq. (24), we obtain

$$
\begin{aligned}
\overline{\mathcal{F}} & =-\omega I_{r e s}+G_{r e s}+\frac{1}{2} g P_{\phi}^{2}-r \bar{\phi}+\varepsilon u F(\bar{\phi}) \\
G_{r e s} & =G_{r e s}\left(x, p_{x}+k I_{r e s}\left(x, p_{x}\right)\right)
\end{aligned}
$$

where

$$
r=\left\{G_{r e s}, I_{r e s}\right\}=\frac{\partial G_{r e s}}{\partial x} \frac{\partial I_{r e s}}{\partial P_{x}}-\frac{\partial G_{r e s}}{\partial P_{x}} \frac{\partial I_{r e s}}{\partial x}
$$

For the sake of simplicity, we omitted the bar over the variables $\phi, p_{x}$, and $x$. Hamiltonian (27) can be viewed as

$$
\overline{\mathcal{F}}=\Lambda+H_{\phi}
$$

Hamiltonian $\Lambda$ describes the slow motion in the $\left(P_{x}, x\right)$ plane:

$$
\Lambda=-\omega I_{r e s}+G_{r e s}\left(x, P_{x}\right)
$$

and Hamiltonian $H_{\phi}$ describes the fast motion in the $\left(P_{\phi}, \phi\right)$ plane:

$$
H_{\phi}=\frac{1}{2} g(x) P_{\phi}^{2}+\varepsilon u(x) F(\phi)-r(x) \phi
$$




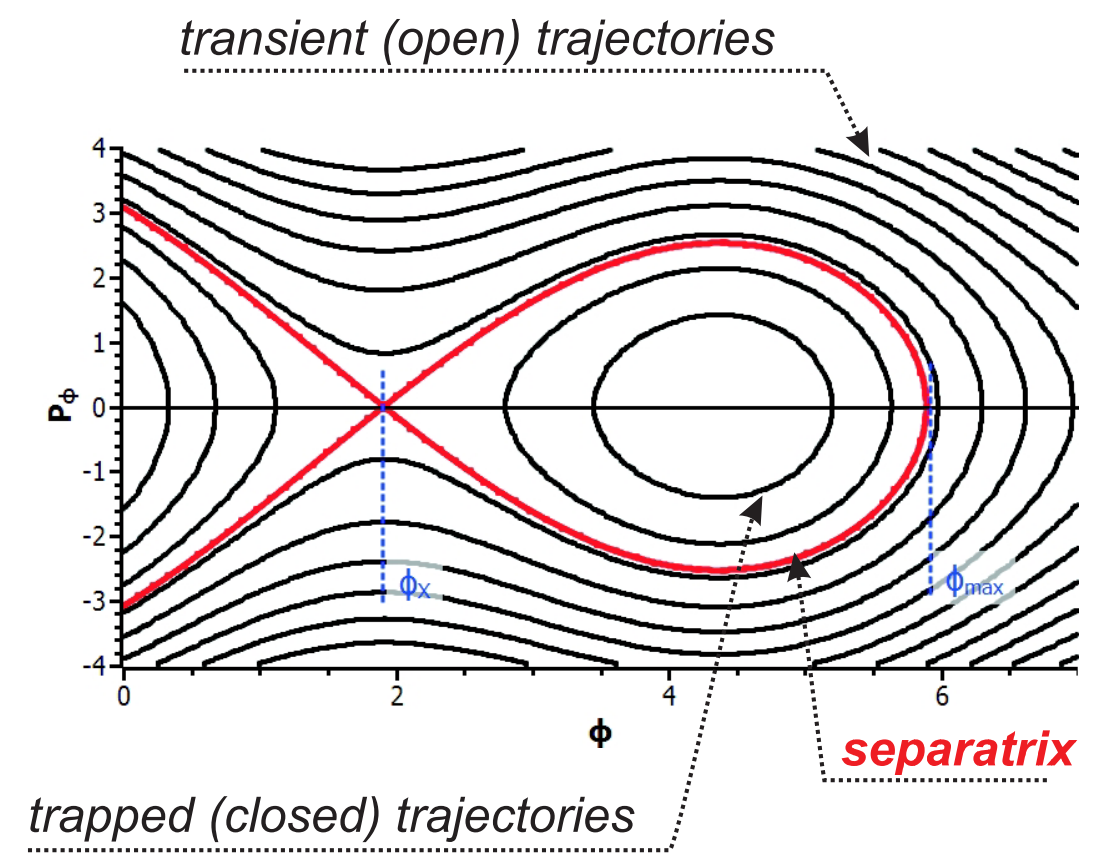

Figure 4: Phase portraits of system (34) for $|\varepsilon u|>|r|$. The red line is a separatrix.

Coefficients in Hamiltonian (31) may depend on $x$ and $P_{x}=P_{\text {res }}(x)$ where $P_{\text {res }}$ is the solution of equation $\Lambda\left(x, P_{x}\right)=$ $h=$ const (see, e.g., Eqs. (19), (20), (30)).

For systems (3) and (5) with $F=\sin \phi$ and $I_{\text {res }}$ given by Eq. (15), we obtain

$$
g=k^{2}, r=-\frac{\Omega^{2}}{k} x
$$

For system (4) with $F=\sin \phi$ and $I_{\text {res }}$ given by Eq. (17), we obtain

$$
g=\frac{\left(1-v_{r e s}^{2}\right)^{3 / 2} k^{2}}{\sqrt{1+\Omega^{2} x^{2}}} r=-\frac{\Omega^{2} x}{k} \frac{1}{\sqrt{1-v_{r e s}^{2}} \sqrt{1+\Omega^{2} x^{2}}}
$$

For Hamiltonians (3), (4), and (5), Eq. (31) can be written as

$$
H_{\phi}=\frac{1}{2} g(x) P_{\phi}^{2}-r(x) \phi+\varepsilon u(x) \sin \phi
$$

Hamiltonian (34) is a classical Hamiltonian of a pendulum with torque. A characteristic phase portrait of $H_{\phi}$ for $r>0$, $|\varepsilon u(x)|>r$ is shown in Fig. 4. There are two types of trajectories: open and closed. The boundary of the region filled with closed trajectories is called the separatrix. Phase points moving along closed trajectories oscillate around the line $P_{\phi}=0$, that defines the resonance $\dot{\phi}=0$.

Section summary. In the current section we showed that multi-scale systems (systems with fast phase) can be studied using the method of averaging. This method is valid away from the resonances - where the rate of change of a fast variable vanishes. We illustrated a way of locating the resonances. We showed how to expand the Hamiltonian in the vicinity of the resonance, to obtain a Hamiltonian of a nonlinear pendulum. The parameters of the resonance Hamiltonian are defined by the coefficients of the system configuration. 


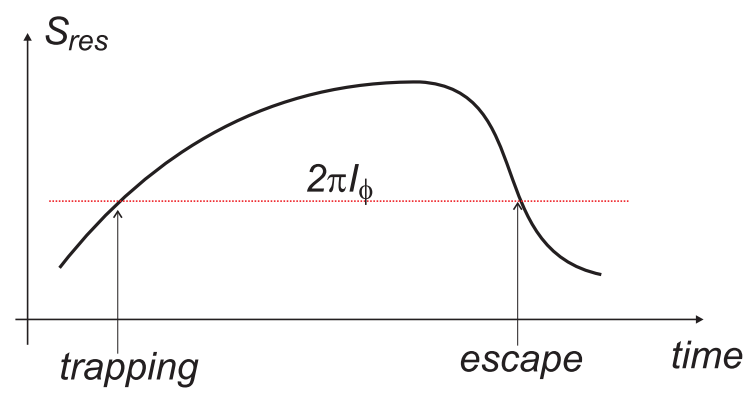

Figure 5: Schematic view of trapping into resonance and escape from resonance.

\section{Trapping (capture) into resonance}

The phase portrait of a typical Hamiltonian (31) is shown in Fig. 4. The curve demarcating regions filled with closed and open trajectories is called the separatrix. Denote by $S_{\text {res }}$ the area bounded by the separatrix in Fig. 4:

$$
S_{\text {res }}=2 \int_{\phi_{x}}^{\phi_{\max }} P_{\phi} d \phi
$$

If the area $S_{\text {res }}$ grows along the particle trajectory, new trajectories can be trapped into this region. The trapping corresponds to a change of type of particle motion: trapped particles oscillate around the resonance $P_{\phi}=0$. Thus, this transition is called trapping (or capture) into resonance.

As trapped particles move along the closed trajectories, the corresponding invariant is conserved

$$
I_{\phi}=\frac{1}{2 \pi} \oint P_{\phi} d \phi=\frac{1}{\pi} \sqrt{\frac{2}{g}} \int_{\phi_{-}}^{\phi_{+}} \sqrt{2 H_{\phi}+r \phi-\varepsilon u \sin \phi} d \phi
$$

where $\phi_{ \pm}$are two solutions of the equation $2 H_{\phi}+r \phi-\varepsilon u \sin \phi=0$. The conservation of $I_{\phi}$ is a consequence of a separation of time scales between $\left(\phi, P_{\phi}\right)$ and $\left(x, P_{x}\right)$.

At the moment of trapping $I_{\phi}=S_{\text {res }} / 2 \pi$. In the trapped motion, $I_{\phi}=$ const and $S_{r e s}\left(x, P_{x}\right)$ changes along the resonant trajectory. Thus, as long as the area $S_{\text {res }}$ grows, the inequality $I_{\phi} \leq S_{\text {res }} / 2 \pi$ does not allow particles to cross the separatrix again and to escape from resonance. For the particle to escape from resonance the area $S_{\text {res }}$ should decrease to its value at the trapping (see a schematics in Fig. 5). Therefore, the $\left(x, P_{x}\right)$ coordinates of trapping and escape are controlled by the shape of the $S_{r e s}\left(x, P_{x}\right)$ curve. In this section, we describe details of the trapped dynamics.

\subsection{Area surrounded by the separatrix}

To define the conditions of trapping, we write an expression for the area $S_{r e s}$ :

$$
S_{\text {res }}=\oint P_{\phi} d \phi=2 \sqrt{\frac{2}{g}} \int_{\phi_{X}}^{\phi_{\max }} \sqrt{H_{\phi}+r \phi-\varepsilon u F(\phi)} d \phi=2 \sqrt{\frac{2|r|}{g}} \int_{\phi_{X}}^{\phi_{\max }} \sqrt{\left(\phi-\phi_{X}\right)-a\left(F(\phi)-F\left(\phi_{X}\right)\right)} d \phi
$$

where

$$
a=\varepsilon u / r
$$

Here $\phi_{X}$ is the coordinate of the saddle point (i.e., a solution of $\left.1-a F^{\prime}(\phi)=0\right), \phi_{\max }$ is a solution of $\left(\phi-\phi_{X}\right)-$ $a\left(F(\phi)-F\left(\phi_{X}\right)\right)=0$ (see Fig. 4). The change of the sign of $r$ corresponds to changing the direction of integration along $\phi$ because $\phi_{X}$ becomes larger than $\phi_{\max }$. In what follows we write the area $S_{\text {res }}$ as

$$
S_{\text {res }}=2 \sqrt{\frac{2|r|}{g}} \times\left\{\begin{array}{lc}
f_{s}(a), & \mathrm{F}(\phi)-\text { periodic } \\
\tilde{f}_{s}(a), & \mathrm{F}(\phi)-\text { nonperiodic }
\end{array}\right.
$$



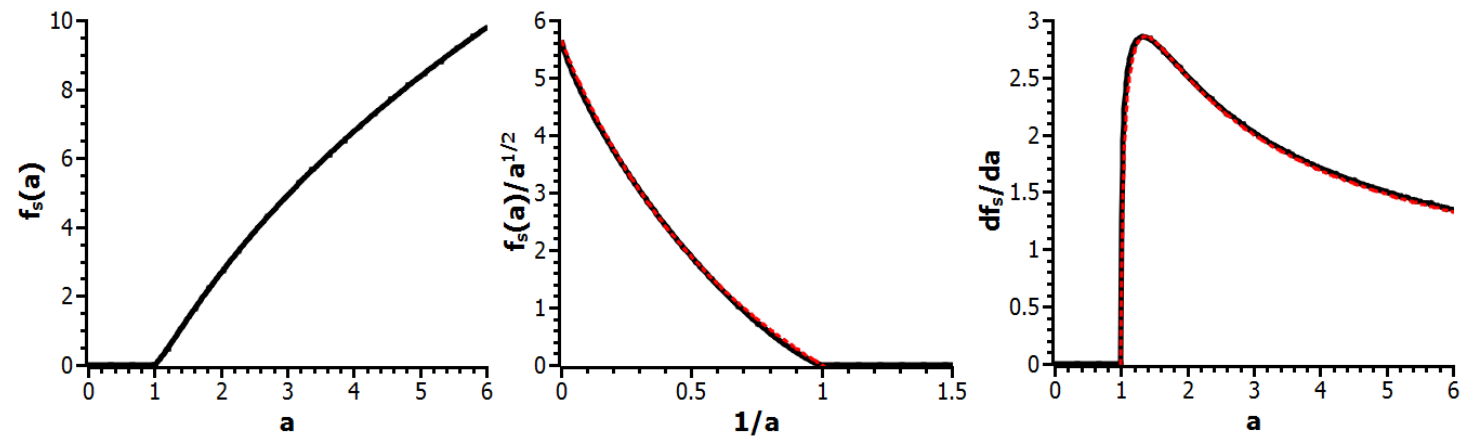

Figure 6: Left panel shows function $f_{s}(a)$ given by Eq. (40). Central panel shows $f_{s}(a) / \sqrt{a}$ as a function of $1 / a$ and approximation (red dashed curve) $f_{s}(a)=8 \sqrt{2 a}(a /(1+a)-1 / 2)$. Right panel shows function $d f_{s}(a) / d a$ and approximation (red dashed curve) $d f_{s}(a) / d a \approx C_{1} \sqrt{a-1} /\left(a^{1.1}-\right.$ $C_{2}$ ) with $C_{1} \approx 3.917$ and $C_{2} \approx 0.58$.

For example, for a system with $F=\sin \phi$, Eq. (39) takes the form

$$
S_{r e s}=2 \sqrt{\frac{2|r|}{g}} \int_{\phi_{X}}^{\phi_{\max }} \sqrt{\left(\phi-\phi_{X}\right)-a\left(\sin \phi-\sin \phi_{X}\right)} d \phi=2 \sqrt{\frac{2|r|}{g}} f_{s}(a)
$$

The profile of $f_{s}(a)$ is shown in Fig. 6 . When $a<1, f_{s}(a)=0$; when $a \rightarrow \infty$, we have $f_{s}(a) \rightarrow 8 \sqrt{a / 2}$ and there is a simple asymptotic expression for $S_{\text {res }}$ :

$$
S_{\text {res }}=16 \sqrt{|\varepsilon u / g|}
$$

Function $f_{s}(a)$ can be reasonably approximated by $f_{s}(a) \approx 8 \sqrt{2 a}(a /(1+a)-1 / 2)$. However, this approximation is not accurate enough to compute the derivative $d f_{s} / d a$. For a better fit, see an example in Fig. 6.

Let us consider behavior of $S_{r e s}$ for systems (3)-(5). For system (3), $u=1$ and we obtain

$$
S_{\text {res }}=\frac{2 \Omega}{k^{3 / 2}} \sqrt{2|x|} f_{s}(a), \quad a=-\frac{\varepsilon k}{x \Omega^{2}}
$$

Profile of $S_{\text {res }}(x)$ is shown in Fig. 7 (left panel). The area $S_{\text {res }}$ differs from zero only for $|x \Omega|<|\varepsilon k u / \Omega|$ and grows $\left(d S_{r e s} / d x>0\right)$ for negative $x$.

For system (4), $u=1$ again and we obtain

$$
S_{\text {res }}=\frac{2 \Omega}{k^{3 / 2}\left(1-v_{\text {res }}^{2}\right)} \sqrt{2|x|} f_{s}(a), \quad a=-\frac{k \varepsilon \sqrt{1-v_{\text {res }}^{2}} \sqrt{1+\Omega^{2} x^{2}}}{\Omega^{2} x}
$$

Profile of $S_{\text {res }}(x)$ is shown in Fig. 7 (central panel). In comparison with $S_{\text {res }}$ given by Eq. (42), area (43) grows with $x$ for large enough $x \Omega$.

For system (5), $u=x$ and we obtain

$$
S_{\text {res }}=\frac{2 \Omega}{k^{3 / 2}} \sqrt{2|x|} f_{s}(a), \quad a=-\frac{\varepsilon k}{\Omega^{2}}=\text { const }
$$

A characteristic profile of $S_{\text {res }}(x)$ is shown in Fig. 7 (right panel). The area $S_{\text {res }}$ grows for positive $x$.

\subsection{Probability of trapping}

The necessary condition for trapping of new particles into resonance at a given location on resonance is the growth of the area $S_{r e s}$ along the resonant trajectories in the slow variables plane, $\left(x, P_{x}\right)$. Not all particles passing through the resonance (resonant particles) are trapped into resonance. The fate of a particular particle is defined by a value of phase $\phi$ near the resonance. This value is defined by the initial (far from the resonance) $\phi$ and can be individually 

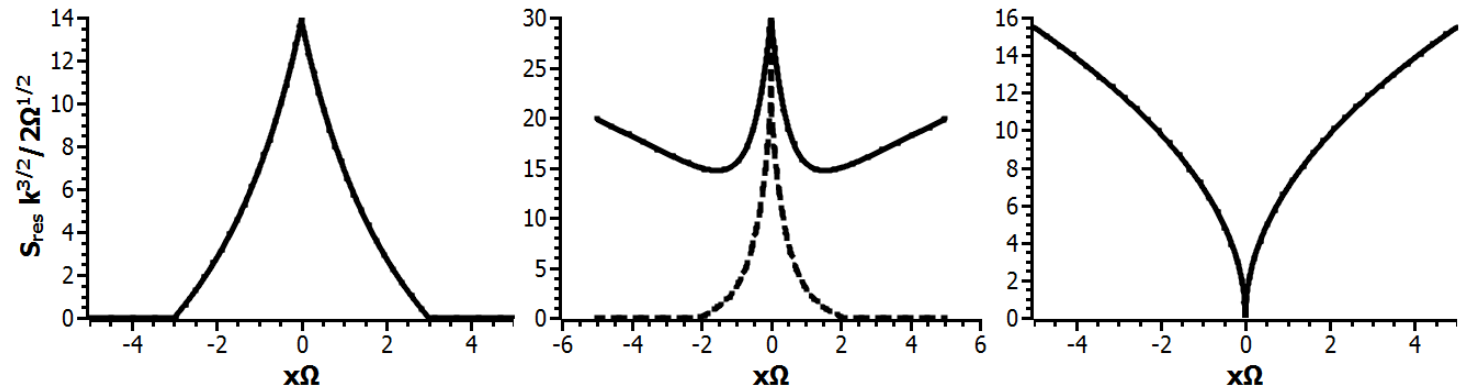

Figure 7: Profiles of $S_{\text {res }}$ from Eq. (42) (left panel), Eq. (43) (central panel), and Eq. (44) (right panel). System parameters are: $\varepsilon k / \Omega=-3$, $v_{\text {res }}=0.8$. The dashed curve (in central panel) shows $S_{\text {res }}$ for Eq. (43) with $\varepsilon k / \Omega<-1 / \sqrt{1-v_{\text {res }}^{2}}$.

calculated for each particle. However, $\phi$ changes fast and even small alternations of the initial particle coordinates can switch the type of motion near the resonance (particle which should not be trapped will be trapped or vice versa). As a result, exactly what particles are to be trapped cannot be realistically predicted. Instead one can compute a relative amount (percentage) of to-be-trapped particles, or, equivalently, the ratio of the phase volume filled by to-be-trapped particles to the entire phase volume filled by resonant particles. This ratio can be called a probability $\Pi$ of trapping during a single passage through resonance, [104]. The to-be-trapped phase volume is equal to $\dot{S}_{r e s} \Delta t$ where $\dot{S}_{\text {res }}$ is a rate of change of $S_{r e s}$, and $\Delta t$ is a time interval. The entire phase volume is a product of the phase flux through the resonance and the same $\Delta t$. Thus, for $\Pi$ we have (see [105]):

$$
\Pi \approx \frac{\left\{S_{\text {res }}, \Lambda\right\}}{\left\{S_{r e s}, \Lambda\right\} / 2+\left|\int_{0}^{2 \pi} \dot{P}_{\phi} d \phi\right|}
$$

and $\Pi=0$ for $\dot{S}_{\text {res }}<0$. Hamiltonian $\Lambda$ in Eq. (45) is defined by Eq. (30). The calculation of $\Pi$ is illustrated in Fig. 8. For Hamiltonian (34), Eq. (45) takes the form:

$$
\Pi=\frac{2\left\{S_{r e s}, \Lambda\right\}}{\left\{S_{r e s}, \Lambda\right\}+4 \pi|r|}
$$

If $S_{r e s}$ changes slowly enough $\left(\dot{S}_{r e s} \ll 4 \pi|r|\right)$, which is the case in almost all systems, we can use approximation

$$
\Pi \approx \frac{\left\{S_{r e s}, \Lambda\right\}}{2 \pi|r|}
$$

For systems (3)-(5) the area $S_{r e s}$ depends only on $x$. Thus

$$
\left\{S_{\text {res }}, \Lambda\right\}=\frac{\partial S_{\text {res }}}{\partial x} \frac{\partial \Lambda}{\partial P_{x}}=\dot{x} \frac{\partial S_{\text {res }}}{\partial x}=P_{\text {res }} \frac{\partial S_{\text {res }}}{\partial x}
$$

For system (3), the area $S_{\text {res }}$ given by Eq. (42), $r$ given by Eq. (32), and we obtain

$$
\Pi \approx \frac{v_{r e s}}{\pi \Omega k^{1 / 2}} \frac{1}{|x|} \frac{\partial}{\partial x}\left(\sqrt{2|x|} f_{s}(a)\right)
$$

Profile of $\Pi(x)$ is shown in Fig. 9(a): positive probabilities correspond to negative values of $x$, where the area $S_{\text {res }}$ increases with $x$ (compare Figs. 7 and 9(a)).

For system (4), $S_{r e s}$ is given by Eq. (43) and $r$ is given by Eq. (33):

$$
\Pi \approx \frac{1}{\pi \Omega k^{1 / 2} \sqrt{1-v_{r e s}^{2}}} \frac{\sqrt{1+\Omega^{2} x^{2}}}{|x|} \frac{\partial}{\partial x}\left(\sqrt{2|x|} f_{s}(a)\right)
$$




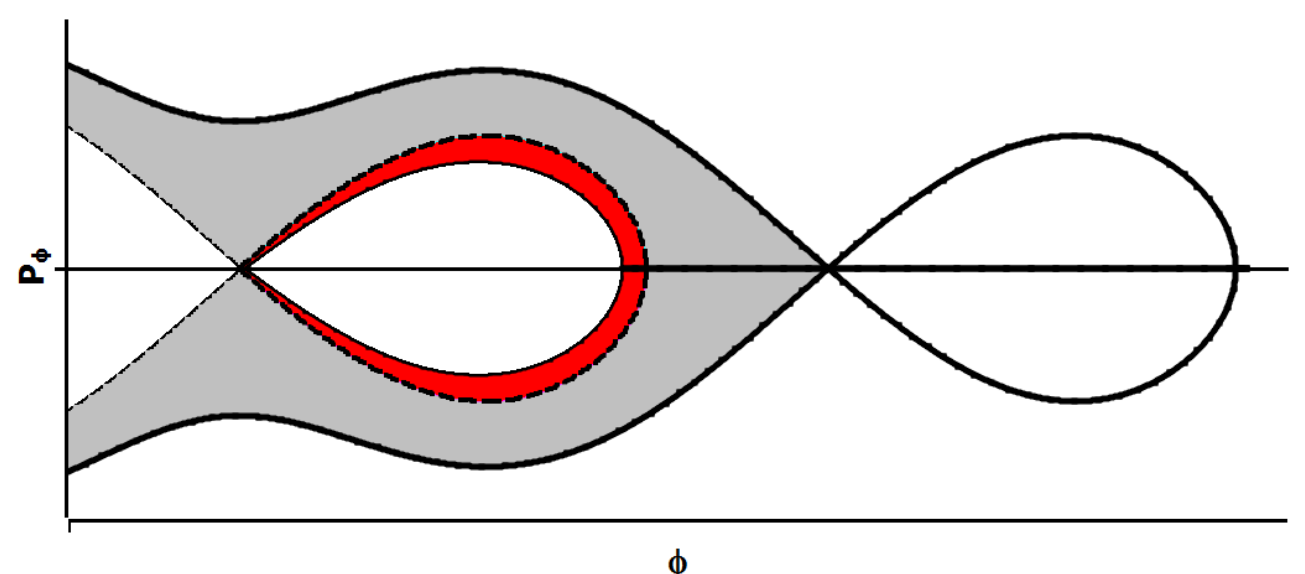

Figure 8: Schematics of the calculation of $\Pi$. The red color shows a change of the area $S_{\text {res }}$ over a time $\sim \Delta t$, the grey color shows a phase space filled by the transient trajectories. The probability of trapping is equal to the ratio of $\Delta S_{r e s} / \Delta t$ to the phase flux through the grey area.

Profile of $\Pi(x)$ is shown in Fig. 9(b). Note that for $|x| \gg 1 / \Omega$, Eq. (43) gives $a=$ const:

$$
a=\frac{k \varepsilon \sqrt{1-v_{r e s}^{2}} \sqrt{1+\Omega^{2} x^{2}}}{\Omega^{2}|x|} \approx \frac{k \varepsilon \sqrt{1-v_{r e s}^{2}}}{\Omega}
$$

and for $|a|>1$ expression (50) takes the form

$$
\Pi \approx \frac{f_{s}(a)}{\sqrt{2} \pi \Omega k^{1 / 2} \sqrt{1-v_{r e s}^{2}}} \frac{\sqrt{1+\Omega^{2} x^{2}}}{|x|^{3 / 2}} \approx \frac{f_{s}(a)}{\sqrt{2} \pi k^{1 / 2} \sqrt{1-v_{r e s}^{2}}} \frac{1}{|x|^{1 / 2}}
$$

Equation (52) shows that $\Pi$ is always positive for large $x$. Thus, new particles can be trapped and trapped particle cannot escape from resonance for large $x$.

For system (5), with $S_{\text {res }}$ given by Eq. (44) and $r$ is given by Eq. (32):

$$
\Pi \approx \frac{f_{s}(a)}{\pi \Omega k^{1 / 2}} \frac{1}{|x|} \frac{\partial}{\partial x}(\sqrt{2|x|})=\frac{f_{s}(a)}{\sqrt{2} \pi \Omega k^{1 / 2}} \frac{1}{|x|^{3 / 2}}
$$

where $a=\varepsilon k / \Omega^{2}=$ const. Profile of $\Pi(x)$ is shown in Fig. 9(c).

\subsection{Non-periodic perturbations}

Equation (45) describes the probability of particle trapping into resonance with a periodic wave. The important property of the resonance particle interaction with such waves is that all the particles crossing the resonance in the slow variable phase plane interact there with the wave, i.e. for all resonant particles the perturbation is described by the same periodic function $F(\phi)$. When the wave is non-periodic, the situation is more complicated. Consider a localized wave, for which $F(\phi) \neq 0$ only over a limited range of $\phi$. For such functions, resonant particles crossing the manifold $\dot{\phi}=0$ can miss the wave: at the moment of crossing the phase $\phi$ can be such that $F=0$. Such resonant particles do not interact with waves. Therefore, for non-periodical waves the trapping probability can be meaningfully calculated only for an ensemble of non-periodical waves sufficiently large to support the resonant wave-particle interaction for all resonant particles. To the best of our knowledge there is no example of such calculations.

\subsection{Dynamics of trapped particles}

Motion of trapped particles in the $\left(x, P_{x}\right)$ plane is described by Hamiltonian (30). Particle momentum in the resonance, $P_{\text {res }}$, is given by Eq. (18). Substituting Eq. (18) into Eq. (8) we obtain the resonant momentum in initial coordinates $\left(x, p_{x}\right)$. 


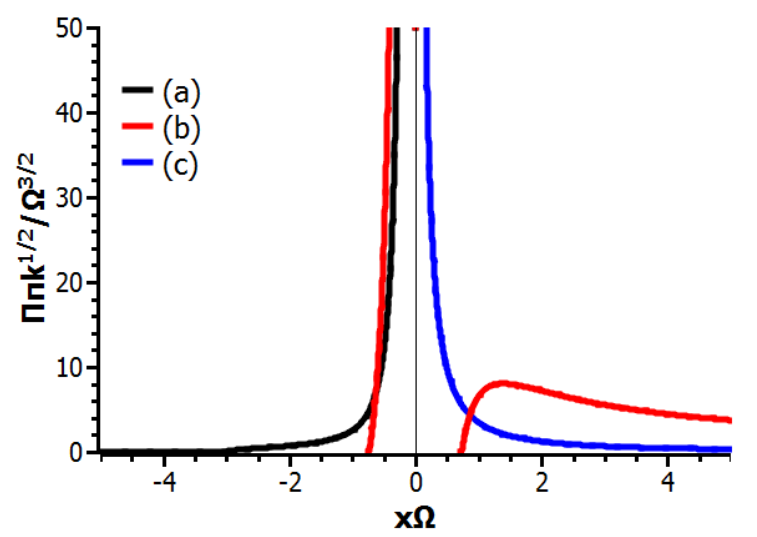

Figure 9: Profiles of $\Pi$ for Eq. (49)(a), Eq. (50)(b), Eq. (53)(c). System parameters are: $\varepsilon k / \Omega^{2}=-3, v_{r e s}=0.8$.

For systems (3) and (5), momentum $p_{x, \text { res }}=v_{\text {res }}=\omega / k$ is conserved, and we obtain that in resonance $x_{\text {res }}=$ $\int p_{x, r e s} d t=v_{r e s} t+x_{0}$. We substitute these expressions into equation for particle energy to get

$$
G_{r e s}=\frac{1}{2} v_{r e s}^{2}+\frac{1}{2} \Omega^{2}\left(v_{r e s} t+x_{0}\right)^{2}
$$

For system (4), the same procedure is based on Eq. (20) and gives

$$
G_{r e s}=\frac{\sqrt{1+\Omega^{2}\left(v_{r e s} t+x_{0}\right)^{2}}}{\sqrt{1-v_{r e s}^{2}}}
$$

Figure 10 illustrates that Eq. (54) can describe both increase and decrease of particle energy. For system (3), trapping is possible only for $x_{\text {trap }}<0$ (see Fig. 9(a)) and the energy decreases with time until $x$ reaches 0 . Then the energy starts increasing and reaches the initial value at $x=-x_{\text {trap }}$. At that moment, the area $S_{\text {res }}$ returns to its value at the moment of trapping (see Fig. 7 (left panel)), and the particle escapes from resonance (see Subsect. 6.1 for more details). The situation is different for system (5), where trapping is possible only for positive $x_{\text {trap }}$ (see Fig. 9(c)). In this case, the energy of trapped particle always increases with time, the area $S_{\text {res }}$ grows, (see Fig. 7 (right panel)), and trapped particles keep accelerating (see Subsect. 6.2). A similar situation takes place for system (4): trapping is possible for positive $x_{\text {trap }}$ when both the energy $G_{\text {res }}$ and the area $S_{\text {res }}$ grow with time (it follows from Eq. (20) that $p_{x, \text { res }}>0$ ), see Figs. 7 (central panel) and 10(curve b). More details about system (4) can be found in Subsect. 7.1.

Section summary. In this section we derive a general expression for the probability of trapping, $\Pi$, which is a relative measure of resonant particles trapped into resonance for a single resonance crossing. We show that trapped particles change their motion significantly and gain energy moving in the resonance with wave. The trapped dynamics is determined by the evolution of the area surrounded by the separatrix, $S_{\text {res }}$.

\section{Scattering on resonance}

Particles passing through resonance change their energy even in absence of trapping. This effect is called scattering on resonance. To describe the energy change for scattered particles, we consider Hamiltonian (9) and write Hamiltonian equation for the momentum $I$ :

$$
\dot{I}=-\frac{\partial \mathcal{F}}{\partial \phi}=-\varepsilon u(x) \cos \phi
$$




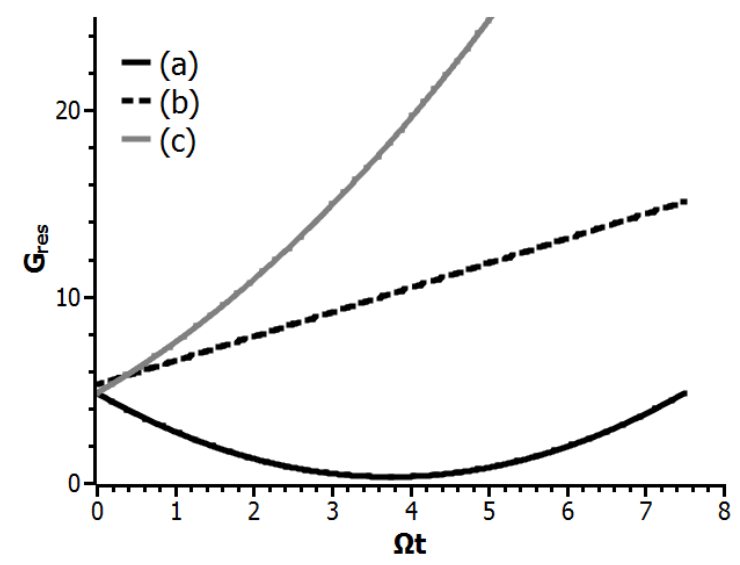

Figure 10: Profiles of $G_{r e s}$ for Eq. (54) with initial negative $x_{0}$ (curve a) and positive $x_{0}$ (curve c), and Eq. (55)(curve b). System parameters are: $\Omega x_{0}=-3(\mathrm{a}), \Omega x_{0}=3(\mathrm{~b}), \Omega x_{0}=3(\mathrm{c}), v_{\text {res }}=0.8$.

where we assumed $F(\phi)=\sin \phi$ like in Hamiltonian (34). Trajectories of a few scattered, or transient, particles in system (31) are shown in Fig. 4. Any such trajectory consists of two symmetrical parts: phase changes from some initial value to $\phi^{*}$ and back. The value of $\phi^{*}$ can be obtained from the equation $P_{\phi}=0$ with Hamiltonian (34):

$$
-r(x) \phi^{*}+\varepsilon u(x) \sin \phi^{*}=H_{\phi}
$$

Therefore, the total change of momentum $I$ for transient particles can be written as

$$
\begin{aligned}
\Delta I & =-2 \int_{-\infty}^{t^{*}}(\varepsilon u \cos \phi) d t=-2 \frac{\varepsilon u}{g} \int_{-\infty}^{\phi^{*}} \frac{\cos \phi}{P_{\phi}} d \phi=-\sqrt{\frac{2}{g}} \varepsilon u \int_{-\infty}^{\phi^{*}} \frac{\cos \phi}{\sqrt{H_{\phi}+r \phi-\varepsilon u \sin \phi}} d \phi \\
& =-\sqrt{\frac{2}{g}} \varepsilon u \int_{-\infty}^{\phi^{*}} \frac{\cos \phi}{\sqrt{r\left(\phi-\phi^{*}\right)-\varepsilon u\left(\sin \phi-\sin \phi^{*}\right)}} d \phi=-\sqrt{\frac{2}{g|r|}} \varepsilon u \int_{-\infty}^{\phi^{*}} \frac{\cos \phi}{\sqrt{2 \pi \theta+\phi-a \sin \phi}} d \phi
\end{aligned}
$$

where we used a notation $2 \pi \theta=a \sin \phi^{*}-\phi^{*}$. Introducing

$$
f_{p}(\theta, a)=\int_{-\infty}^{\phi^{*}} \frac{\cos \phi}{\sqrt{2 \pi \theta+\phi-a \sin \phi}} d \phi
$$

we get $\Delta I=-\sqrt{2 / g|r|} \varepsilon u f_{p}(\theta, a)$. Function $f_{p}(\theta, a)$ is $2 \pi$-periodic in $\theta$ and its profile is shown in Fig. 11 for three values of $a$. For a particular particle, fast oscillations of $\phi$ result in a significant change of $\theta$ even for small changes of the initial energy: two very close initial energies can correspond to very different $\phi^{*}$. Thus, it is convenient to use statistical properties of $f_{p}(\theta, a)$, which characterize the dynamics of an ensemble of particles with different values of $\theta$. Assuming a uniform distribution of $\theta$ (see a numerical verification of this assumption in [106]), we get

$$
\begin{aligned}
\langle\Delta I\rangle_{\theta} & =\int_{0}^{1} \Delta I d \theta=-\sqrt{\frac{2}{g|r|}} \varepsilon u\left\langle f_{p}\right\rangle_{\theta} \\
\operatorname{Var}(\Delta I) & =\int_{0}^{1} \Delta I^{2} d \theta-\langle\Delta I\rangle_{\theta}^{2}=\frac{2}{g|r|}(\varepsilon u)^{2} \operatorname{Var}\left(f_{p}\right)
\end{aligned}
$$



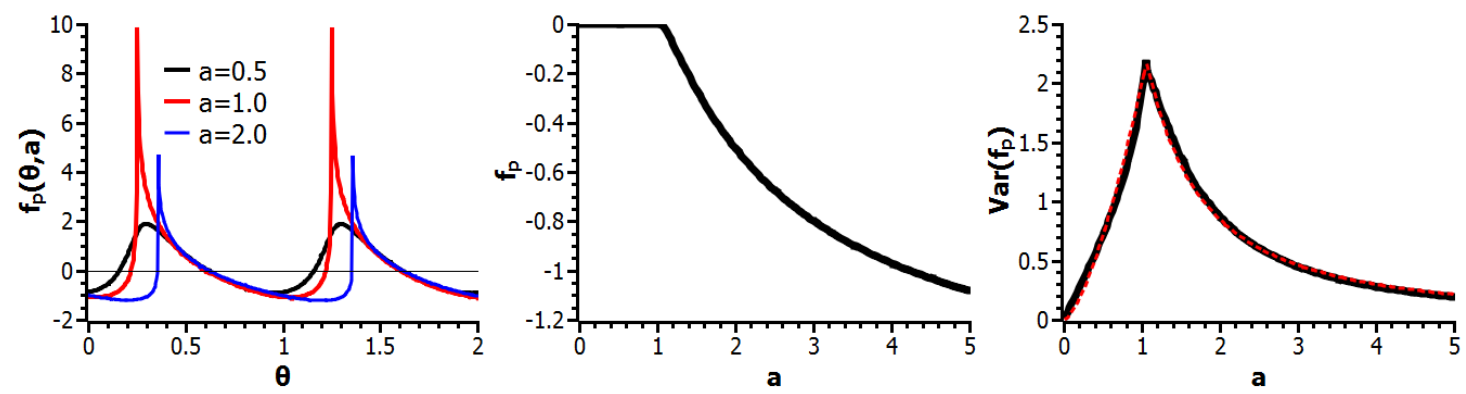

Figure 11: Profiles of functions $f_{p}(\theta, a)$ (left panel), $f_{p}(a)=\left\langle f_{p}\right\rangle$ (central panel), $\operatorname{Var}\left(f_{p}\right)$ (right panel). Red dashed curves shows approximation: where $\operatorname{Var}\left(f_{p}\right)=2 a^{3 / 2}$ for $|a|<a_{*}$ and $\operatorname{Var}\left(f_{p}\right)=2.4 a^{-3 / 2}$ for $|a|>a_{*}, a_{*} \approx 1.06$.

Figure 11 shows profiles of $f_{p}(a)=\left\langle f_{p}\right\rangle_{\theta}$ and $\operatorname{Var}\left(f_{p}\right)$. From now on, we omit $\langle\ldots\rangle_{\theta}$, as in the present review, and in almost all of the publications cited here, the function $f_{p}(\theta, a)$ is not used. The function $f_{p}$ is zero for $a<1$, whereas $\operatorname{Var}\left(f_{p}\right)$ has a maximum at $a=1$ (we can approximate $\operatorname{Var}\left(f_{p}\right)$ by a simple function shown in Fig. 11).

Equations (60) describe changes of momentum $I$ due to scattering. This momentum is a slowly changing variable in autonomous Hamiltonian (9): $\mathcal{F}\left(I, x, P_{x}\right)=$ const. Thus, $I$ variation results in variation of other slow variables, $x$ and $P_{x}$ : scattering on resonance leads to variations of all slowly varying system variables. Conservation of Hamiltonian $\mathcal{F}$ results in a direct relation between the momentum change $\Delta I$ is and the energy change $\Delta h=-\omega \Delta I$. Thus Eqs. (60) describe particle acceleration/deceleraton due to scattering. Similarly, the momentum $p_{x}$ is related to $I$ through the resonant condition: $k p_{x}-\omega=\left(I-I_{\text {res }}\right) g$. And finally $\Delta h$ and $\Delta p_{x}$ are related via unperturbed Hamiltonian (1): $\left.\Delta h=\left(\partial G / \partial p_{x}\right)_{\mathcal{F}=\text { const }} \Delta p_{x}\right)$.

Hamiltonian equations for Hamiltonian (9) yield $I-I_{\text {res }}=\dot{\phi} / g$. As $\dot{\phi}$ can be defined from Eq. (2), we obtain

$$
k p_{x}-\omega=\left(I-I_{\text {res }}\right) g
$$

Combination of Eqs. (60) and (61) defines $\Delta p_{x}$ for scattered particles:

$$
\begin{aligned}
\left\langle\Delta p_{x}\right\rangle & =\frac{g\langle\Delta I\rangle_{\theta}}{k}=-\sqrt{\frac{2 g}{|r| k^{2}}} \varepsilon u f_{p} \\
\operatorname{Var}\left(\Delta p_{x}\right) & =\frac{g^{2}}{k^{2}} \operatorname{Var}(\Delta I)=\frac{2 g}{|r| k^{2}}(\varepsilon u)^{2} \operatorname{Var}\left(f_{p}\right)
\end{aligned}
$$

Here $\Delta p_{x}$ is essentially a change of $p_{x}$ after a passage through resonance (more accurately, $\Delta p_{x}$ is a difference between an actual $p_{x}$ value after a passage and $p_{x}$ at the same time moment in the system without a wave). For systems (3) and (5), Eq. (62) can be written as (see Eq. (32)):

$$
\begin{aligned}
\left\langle\Delta p_{x}\right\rangle & =-\sqrt{\frac{2}{|x| k}} \frac{k \varepsilon u}{\Omega} f_{p} \\
\operatorname{Var}\left(\Delta p_{x}\right) & =\frac{2}{|x| k}\left(\frac{k \varepsilon u}{\Omega}\right)^{2} \operatorname{Var}\left(f_{p}\right)
\end{aligned}
$$

where $u=1$ for system (3) and $u=|x|$ for system (5). For system (4), Eq. (62) takes the form (see Eq. (33)):

$$
\begin{aligned}
\left\langle\Delta p_{x}\right\rangle & =-\left(1-v_{r e s}^{2}\right)^{3 / 2} \sqrt{\frac{2}{|x| k}} \frac{k \varepsilon}{\Omega} f_{p} \\
\operatorname{Var}\left(\Delta p_{x}\right) & =\left(1-v_{r e s}^{2}\right)^{3} \frac{2}{|x| k}\left(\frac{k \varepsilon}{\Omega}\right)^{2} \operatorname{Var}\left(f_{p}\right)
\end{aligned}
$$

Equations (62) show that scattering on resonance results in variation of particle momentum (equivalently, in variation of particle energy). For systems with $a>1$, this variation contains both a regular change $(d r i f t)\left\langle\Delta p_{x}\right\rangle \neq 0$ and 
diffusive variations. For $a<1,\left\langle\Delta p_{x}\right\rangle=0$ and only diffusive variations are present. This diffusion leads to $p_{x} \sim$ $\sqrt{\operatorname{Var}\left(\Delta p_{x}\right) t} \sim \sqrt{t / k}$ variation with $\operatorname{Var}\left(\Delta p_{x}\right) \sim 1 / k \ll 1$, whereas regular change $\left\langle\Delta p_{x}\right\rangle \sim 1 / \sqrt{k}$ leads to variation $p_{x} \sim t / \sqrt{k}[107]$.

The number of resonant interactions (resonance crossings) for one period of slow variables may be different for different system configurations (slow phase portrait and the shape of the resonance manifold). In terms of the energy (momentum) change due to scattering on resonance, there are two main types of systems with $a>1$. In systems of the first type occurring when there is a certain symmetry of the slow phase portrait, mean changes of energy (momentum) for one period of slow variable oscillations compensate each other. In this case, particle scattering leads to diffusion without drift. In systems of the second type, an asymmetry of the slow variable phase portrait results in the net energy (momentum) change, causing the drift in energy.

Section summary. In this section we described the energy change of a particle during the scattering on resonance. If the resonant system contains a separatrix $\left(S_{r e s} \neq 0\right)$, there is a finite mean energy change due to scattering, and multiple scatterings lead to a directed acceleration/deceleration of particles. In absence of the separatrix, these is no mean energy change, and the energy change over multiple scatterings is diffusive.

\section{Evolution of a particle ensemble}

This section explains how effects of scattering and trapping can be included into kinetic equations describing the long-time evolution of particle distribution functions in the phase space. In Subsect. 5.1 we consider diffusion due to scattering and demonstrate that for different systems this diffusion can be limited or unlimited. In Subsect. 5.3 we discuss possible approaches to include trapping effects into kinetic equations.

\subsection{Diffusive scattering and correlations}

This subsection discusses diffusion in systems where the average drift due to scattering (see Eq. (58)) is absent. We consider two examples to demonstrate two distinct cases: when diffusion leads to an unlimited acceleration and when the diffusive acceleration is limited. The only significant difference between these quite similar plasma systems is that in the first case we deal with a constant frequency of particle oscillations in absence of wave, and in the second case the frequency depends on particle energy. This difference defines the long-term dynamics of resonant particles. In the system with a constant frequency, at high energies the consecutive scatterings become correlated, and the energy diffusion stops. In the energy-depending frequency, a correlation between two successive resonant interactions is destroyed and the diffusion is unlimited [e.g., 108].

\subsubsection{System with a constant frequency}

We start with system (5) described by following Hamiltonian (see [109]):

$$
\begin{aligned}
H & =\frac{1}{2} p_{x}^{2}+\frac{1}{2} \Omega^{2} x^{2}+\varepsilon x \sin \phi \\
\phi & =k x-\omega t
\end{aligned}
$$

where the frequency of particle oscillations $\Omega=$ const. Hamiltonian equations for this system are

$$
\begin{aligned}
& \dot{x}=p_{x} \\
& \dot{p}_{x} \approx-\Omega^{2} x-\varepsilon k x \cos \phi
\end{aligned}
$$

In Eq. (66), we omitted the term $\sim \varepsilon \cos \phi \ll k x \varepsilon \cos \phi$. The equation for $\phi$ is

$$
\ddot{\phi} / k=-\Omega^{2} x-\varepsilon k x \cos \phi
$$

This equation corresponds to the following Hamiltonian (cf. Eq. (34)):

$$
H_{\phi}=\frac{1}{2} k^{2} P_{\phi}^{2}+\frac{\Omega^{2} x}{k} \phi+\varepsilon x \sin \phi
$$


where the momentum $P_{\phi}=\dot{\phi} / k^{2}$ is conjugate to $\phi$. The derivation of Hamiltonian (68) is totally similar to that of Hamiltonian (34). The variation of particle energy with time is (see Eq. (65))

$$
\frac{d}{d t} h=\varepsilon \omega x \cos \phi
$$

Thus, for the change of energy for one resonance crossing we have

$$
\Delta h=2 \varepsilon \omega x \int_{-\infty}^{t^{*}} \cos \phi d t=\frac{2 \varepsilon \omega|x|}{k^{2}} \int_{-\infty}^{\phi^{*}} \frac{\cos \phi}{P_{\phi}} d \phi=v_{\text {res }} \sqrt{2 \varepsilon|x|} \int_{-\infty}^{\phi^{*}} \frac{\sqrt{a} \cos \phi}{\sqrt{\left(\phi^{*}-\phi\right)+a\left(\sin \phi^{*}-\sin \phi\right)}} d \phi
$$

where $v_{\text {res }}=\omega / k, a=k \varepsilon / \Omega^{2}, t^{*}$ is the moment when a particle crosses the resonance $\dot{\phi}=0$ ( $\phi^{*}$ is a corresponding phase value). The change $\Delta h$ depends on $\phi^{*}$, and can be treated as a random variable. We consider $a<1$, therefore $f_{p}(a)=0$ (see Sect. 4).

To analyze how the energy changes with time for an ensemble of particles, we consider the geometry of the motion of the particle. In the leading approximation, a phase point in the $\left(\Omega x, p_{x}\right)$ plane moves along a Larmor circle with a radius of $\sqrt{2 h}$. Particles cross the resonance line $p_{x}=\dot{x}=v_{\text {res }}$ if $h>v_{\text {res }}^{2} / 2$. The time between two consecutive intersections is $2 \arccos \left(v_{\text {res }} / \sqrt{2 h}\right) / \Omega$. The wave phase gain over this time is

$$
\Delta \phi=\int(k \dot{x}-\omega) d t=\frac{2 k}{\Omega} \sqrt{2 h-v_{r e s}^{2}}-2 \frac{\omega}{\Omega} \arccos \left(v_{r e s} / \sqrt{2 h}\right)
$$

When the energy is not too high, this phase gain can be considered as a random variable due to energy changes $\Delta h$. Since $k$ and $\omega$ are large, a small change in $h$ leads to a large change in the phase gain. For this reason, the phase values at successive passages through resonance can be treated as independent random variables. Accumulation of multiple energy changes $\Delta h$ given by Eq. (70) results in diffusion: the sum of random changes $\Delta h$ is equal to zero, but the variance of $\Delta h$ is finite. The energy grows until the assumption of independence of $\Delta \phi$ is valid. However, as the energy increases, the phase gain between successive passages through resonance becomes more deterministic

$$
\Delta h \frac{\partial \Delta \phi}{\partial h} \sim h^{-1 / 4} \rightarrow 0
$$

Therefore, the resonant particle motion becomes regular and the diffusion stops. This result can be independently obtained from the KAM theory [104], which predicts that there is an energy $h^{*}$ beyond which the diffusion vanishes. Thus, in systems with constant frequency of unperturbed particle oscillations the diffusion is limited.

\subsubsection{System with a frequency depending on energy}

The second system considered in this subsection is a relativistic analog of system (65). Hamiltonian of this system resembles Hamiltonian (4) but with a more complicated wave amplitude [110]:

$$
\begin{aligned}
H & =\gamma+\varepsilon x \gamma^{-1} \sin \phi \\
\gamma & =\sqrt{1+p_{x}^{2}+\Omega^{2} x^{2}} \\
\phi & =k x-\omega t
\end{aligned}
$$

The factor $\gamma^{-1}$ comes from the expansion of the initial Hamiltonian for a relativistic particle interacting with an electromagnetic wave [110]. The corresponding Hamiltonian equations can be written as

$$
\begin{aligned}
\dot{x} & =\frac{p_{x}}{\gamma} \\
\dot{p}_{x} & \approx-\frac{\Omega^{2}}{\gamma} x-\frac{\varepsilon}{\gamma} k x \cos \phi \\
\phi & =k x-\omega t
\end{aligned}
$$


Frequency of particle oscillations in the $\left(x, p_{x}\right)$ plane, $\sim \Omega / \gamma$, depends on particle energy. Equations $(33,34)$ define $H_{\phi}$ near the resonance:

$$
\begin{aligned}
H_{\phi} & =\frac{1}{2} g(x) P_{\phi}^{2}+r(x) \phi-\frac{\varepsilon}{\gamma_{r e s}} x \sin \phi \\
g & =\frac{\left(1-v_{r e s}^{2}\right)^{3 / 2} k^{2}}{\sqrt{1+\Omega^{2} x^{2}}} \\
r & =-\frac{\Omega^{2} x}{k} \frac{1}{\sqrt{1-v_{r e s}^{2}} \sqrt{1+\Omega^{2} x^{2}}}
\end{aligned}
$$

where $v_{\text {res }}=\omega / k$, the momentum $P_{\phi}=\dot{\phi} / g$ is conjugate to $\phi$, and

$$
\gamma_{\text {res }}=\frac{\sqrt{1+\Omega^{2} x^{2}}}{\sqrt{1-v_{r e s}^{2}}}
$$

Following (38), we have

$$
a=-\frac{\varepsilon x}{\gamma_{r e s} r_{0} k}=\frac{\varepsilon\left(1-v_{r e s}^{2}\right)}{\Omega^{2}}
$$

When $a<1$, the particle energy changes at the resonance due entirely to scattering: $\langle\Delta h=0\rangle$ (see Sect 4 ), but the dispersion is non-zero. Thus the particle energy diffuses.

According to Hamiltonian (73), the time derivative of energy is

$$
\frac{d}{d t} h=\frac{\varepsilon \omega x}{\gamma_{r e s}} \cos \phi
$$

Taking into account that $\dot{\phi}=g P_{\phi}$, we find for the variation of the energy at one resonance crossing (cf. Eq. (70))

$$
\Delta h=\sqrt{\frac{2 \varepsilon|x| \omega^{2}}{g \gamma_{r e s}}} \int_{-\infty}^{\phi^{*}} \frac{\sqrt{a} \cos \phi}{\sqrt{\phi+a \sin \phi-\phi^{*}-a \sin \phi^{*}}} d \phi=\frac{v_{r e s} \sqrt{2 \varepsilon|x|}}{\sqrt{1-v_{r e s}^{2}}} \int_{-\infty}^{\phi^{*}} \frac{\sqrt{a} \cos \phi}{\sqrt{\phi+a \sin \phi-\phi^{*}-a \sin \phi^{*}}} d \phi
$$

where $\phi^{*}$ and $x$ are the values of $\phi, x$ at the crossing of the resonant surface by an unperturbed trajectory with energy $h$. From Hamiltonian equations (74), frequency of particle rotation $\sim \Omega / \gamma$. Thus, the time between two successive crossings is of order $\gamma$. Integrating equations of motion, the difference of phases at the first and the second resonance crossings is $\phi_{*}^{(2)}-\phi_{*}^{(1)} \sim k \gamma$ (cf. Eq. (71)). Thus, a small variation $\delta \phi_{*}^{(1)}$ of the phase $\phi_{*}^{(1)}$ results in a variation of the energy change $\delta(\Delta h) \sim \sqrt{\gamma / k} \delta \phi_{*}^{(1)}$ at the first resonance crossing. The resulting change $\delta \phi_{*}^{(2)}$ is large:

$$
\delta \phi_{*}^{(2)}=\delta(\Delta h) \frac{\partial}{\partial \gamma}\left(\phi_{*}^{(2)}-\phi_{*}^{(1)}\right) \sim \delta(\Delta h) k \sim \phi_{*}^{(2)} \sqrt{k \gamma} \gg \phi_{*}^{(2)}
$$

Therefore, the values of phase at successive resonance crossings are statistically independent. Hence, the changes in the particles energy at the resonance produce a diffusive variation of the energy and its unlimited stochastic growth. Which is different from nonrelativistic system (65) where energy diffusion slows down and finally stops at large enough energies.

Two examples (Hamiltonians (65) and (73)) demonstrate the importance of the energy dependence of the frequency of unperturbed particle motion (in absence of waves) for resonant wave-particle interaction. In realistic plasma systems this frequency can rarely be assumed to be constant, but it is the case in many simplified models.

\subsection{Relation between trapping and scattering}

In this subsection we describe the relationship between the average change of momentum on scattering, $\langle\Delta I\rangle$, and the probability of trapping, $\Pi$. We repeat derivations from [111] and then provide a new expression for $\langle\Delta I\rangle$. 
Consider Hamiltonian (34):

$$
H_{\phi}=\frac{1}{2} g P_{\phi}^{2}+r \phi-\varepsilon u \sin \phi
$$

where the evolution of slow variables $\left(x, P_{x}\right)$ is described by the Hamiltonian $\Lambda\left(x, P_{x}\right)$. The change of the momentum $I$ due to scattering on resonance is given by Eq. (58):

$$
\Delta I=-\sqrt{\frac{2}{g|r|}} \varepsilon u \int_{-\infty}^{\phi^{*}} \frac{\cos \phi}{\sqrt{h_{\phi}-\phi+a \sin \phi}} d \phi
$$

where $h_{\phi}=a \sin \phi^{*}+\phi^{*}$. The average $\langle\Delta I\rangle$ change is

$$
\langle\Delta I\rangle=-\frac{1}{2 \pi} \sqrt{\frac{2|r|}{g}} \int_{-\infty}^{\phi^{*}} d \phi \int_{0}^{2 \pi} \frac{a \cos \phi d h_{\phi}}{\sqrt{h_{\phi}-\phi+a \sin \phi}}
$$

We introduce $\tilde{h}_{\phi}$ as

$$
\tilde{h}_{\phi}=\left\{\begin{array}{cc}
0, & \phi<\phi_{X} \\
\phi-a \sin \phi & \phi>\phi_{X}
\end{array}\right.
$$

where $\phi_{X}$ is a solution of the equations $a \cos \phi=1, a \sin \phi>0$, and $h_{X}=-\phi_{X}+a \sin \phi_{X}$ (see Fig. 4). Then we separate integral (83) into two parts:

$$
\int_{-\infty}^{\phi^{*}} \int_{0}^{2 \pi} \frac{a \cos \phi d h_{\phi} d \phi}{\sqrt{h_{\phi}-\phi+a \sin \phi}}=\int_{-\infty}^{\phi_{X}+2 \pi} \int_{\tilde{h}_{\phi}+h_{X}}^{2 \pi+h_{X}} \frac{a \cos \phi d h_{\phi} d \phi}{\sqrt{h_{\phi}-\phi+a \sin \phi}}-\int_{\phi_{X}}^{\phi_{\max }} \int_{\tilde{h}_{\phi}+h_{X}}^{h_{X}} \frac{a \cos \phi d h_{\phi} d \phi}{\sqrt{h_{\phi}-\phi+a \sin \phi}}
$$

where $\phi_{\max }$ is a solution of equation $\phi_{X}-a \sin \phi_{X}-\phi+a \sin \phi=0$. Then we consider the first integral from Eq. (85):

$$
\begin{aligned}
\int_{-\infty}^{\phi_{X}+2 \pi} \int_{\tilde{h}_{\phi}+h_{X}}^{2 \pi+h_{X}} \frac{a \cos \phi d h_{\phi} d \phi}{\sqrt{h_{\phi}-\phi+a \sin \phi}} & =2 \int_{-\infty}^{\phi_{X}+2 \pi} a \cos \phi\left(\sqrt{2 \pi+h_{X}-\phi+a \sin \phi}-\sqrt{\tilde{h}_{\phi}+h_{X}-\phi+a \sin \phi}\right) d \phi \\
& =2 \int_{-\infty}^{\phi_{X}} a \cos \phi\left(\sqrt{2 \pi+h_{X}-\phi+a \sin \phi}-\sqrt{h_{X}-\phi+a \sin \phi}\right) d \phi \\
& +2 \int_{\phi_{X}}^{\phi_{X}+2 \pi} a \cos \phi \sqrt{2 \pi+h_{X}-\phi+a \sin \phi} d \phi \\
& =\lim _{N \rightarrow \infty} 2 \int_{\phi_{X}+2 \pi}^{\phi_{X}} a \cos \phi\left(\sqrt{2 \pi+h_{X}-\phi+a \sin \phi}-\sqrt{h_{X}-\phi+a \sin \phi}\right) d \phi \\
& +2 \int_{\phi_{X}}^{\phi_{X}} a \cos \phi \sqrt{2 \pi+h_{X}-\phi+a \sin \phi} d \phi \\
& =-2 \int_{\phi_{X}-2 \pi}^{\phi_{X}} a \cos \phi \sqrt{h_{X}-\phi+a \sin \phi} d \phi+2 \int_{\phi_{X}}^{\phi_{X}+2 \pi} a \cos \phi \sqrt{2 \pi+h_{X}-\phi+a \sin \phi} d \phi=0
\end{aligned}
$$

where we took into account that integral (82) is periodic over $h_{\phi}$ (see Sect. 4). The second integral in Eq. (85) is

$$
\int_{\phi_{X}}^{\phi_{\max }} \int_{\tilde{h}_{\phi}+h_{X}}^{h_{X}} \frac{a \cos \phi d h_{\phi} d \phi}{\sqrt{h_{\phi}-\phi+a \sin \phi}}=2 \int_{\phi_{X}}^{\phi_{\max }} a \cos \phi \sqrt{h_{X}-\phi+a \sin \phi} d \phi
$$




$$
=2 \int_{\phi_{X}}^{\phi_{\max }}(a \cos \phi-1) \sqrt{h_{X}-\phi+a \sin \phi} d \phi+2 \int_{\phi_{X}}^{\phi_{\max }} \sqrt{h_{X}-\phi+a \sin \phi} d \phi=2 \int_{\phi_{\max }}^{\phi_{X}} \sqrt{h_{X}+\phi-a \sin \phi} d \phi
$$

We substitute Eqs. $(86,87)$ into Eq. (83)

$$
\langle\Delta I\rangle=-\frac{1}{\pi} \sqrt{\frac{2|r|}{g}} \int_{\phi_{X}}^{\phi_{\max }} \sqrt{\left(\phi_{X}-\phi\right)+a\left(\sin \phi-\sin \phi_{X}\right)} d \phi
$$

Comparing Eq. (88) and Eq. (40), we obtain:

$$
\langle\Delta I\rangle=-\frac{1}{\pi} \sqrt{\frac{2|r|}{g}} \int_{\phi_{X}}^{\phi_{\max }} \sqrt{\left(\phi-\phi_{X}\right)-a\left(\sin \phi-\sin \phi_{X}\right)} d \phi=\frac{1}{\pi} \sqrt{\frac{2|r|}{g}} f_{s}(a)=-\frac{S_{r e s}}{2 \pi}
$$

Thus, the average change of the momentum is proportional to the area $S_{\text {res }}$. Comparison of Eq. (40), Eq. (58), and Eq. (89) yields a relation between $f_{s}(a)$ and $f_{p}(a)=\left\langle f_{p}\left(h_{\phi}, a\right)\right\rangle_{h_{\phi}}$ :

$$
f_{p}(a)=-2 f_{s}(a) / a
$$

Let $V=\langle\Delta I\rangle$ be a velocity of drift in the momentum space. This velocity depends on slow variables $\left(x, P_{x}\right)$ along the resonance trajectory, i.e, we can write $V\left(x, P_{x}\right)=V\left(x, P_{\text {res }}(x)\right)=V(x)=V\left(I_{\text {res }}\right)$ where $I_{\text {res }}=I_{\text {res }}\left(x, P_{\text {res }}\right)$ and $P_{\text {res }}(x)$ are defined by Eqs. (30) and (14). Therefore, we can consider function $V(I)$ where $I=I_{\text {res }}$ in the resonance. The gradient $d V / d t$ along the resonant trajectory is

$$
\frac{d V}{d t}=\{V, \Lambda\}=\frac{d V}{d I} \frac{d I}{d t}=\frac{d V}{d I}\left\{I_{r e s}, \Lambda\right\}=\frac{d V}{d I} r
$$

where $r=\left\{I_{r e s}, \Lambda\right\}$, see Eq. (28). Comparing Eq. (89) and Eq. (47), we obtain

$$
\frac{d V}{d I}=-\frac{\left\{S_{r e s}, \Lambda\right\}}{2 \pi r}=-\Pi
$$

Equation (92) gives a straightforward relation between probability of trapping $\Pi$ and velocity of drift $V$.

\subsection{Kinetic equation for trapped and transient particles}

Using relation (92) for $V$ and $\Pi$, we will derive an evolution equation for the distribution function of particles interacting with waves. For the sake of simplicity, we focus on a simple but rather general $1 \frac{1}{2}$ degrees of freedom system with the Hamiltonian $H=G(\tau, I)+\varepsilon u(\tau, I) \cos \phi$. Here $(\phi, I)$ are conjugate variables, with $I$ being the normalized particle momentum. In this system the phase $\phi$ changes at a rate $\sim 1$ (much faster than the slow time $\tau=\varepsilon t$ ). The resonant condition $\dot{\phi}=\partial G / \partial I=0$ has a solution $I=I_{\text {res }}(\tau)$. Expansion around $I_{\text {res }}$ gives the following Hamiltonian

$$
\mathcal{F}=\Lambda+\frac{1}{2} g\left(I-I_{\text {res }}\right)^{2}+\varepsilon u \cos \phi
$$

where $\Lambda(\tau)=G\left(\tau, I_{\text {res }}(\tau)\right), g(\tau)=\left(\partial^{2} G / \partial I^{2}\right)_{I=I_{\text {res }}}, u(\tau)=u\left(\tau, I_{\text {res }}(\tau)\right)$. We introduce new variable $P_{\phi}=\left(I-I_{\text {res }}\right)$ (see Eq. (22)) and rewrite the new Hamiltonian $H_{\phi}=\mathcal{F}-\Lambda$ as

$$
H_{\phi}=\frac{1}{2} g P_{\phi}^{2}+r \phi+\varepsilon u \cos \phi
$$

where $r=d I_{\text {res }} / d t$ and $\left(\phi, P_{\phi}\right)$ are conjugate variables.

For the sake of definiteness we consider $r>0$ and $g>0$. For $a(\tau)=\varepsilon u / r>1$ the phase portrait of Hamiltonian (94) contains a region filled with closed trajectories. The area of this region, $S_{r e s}$, and the probability of trapping, $\Pi$, are both small, $\sim \sqrt{\varepsilon}$. Once trapped, particles move in resonance with the wave until the value of $S_{\text {res }}(\tau)$ returns to its 


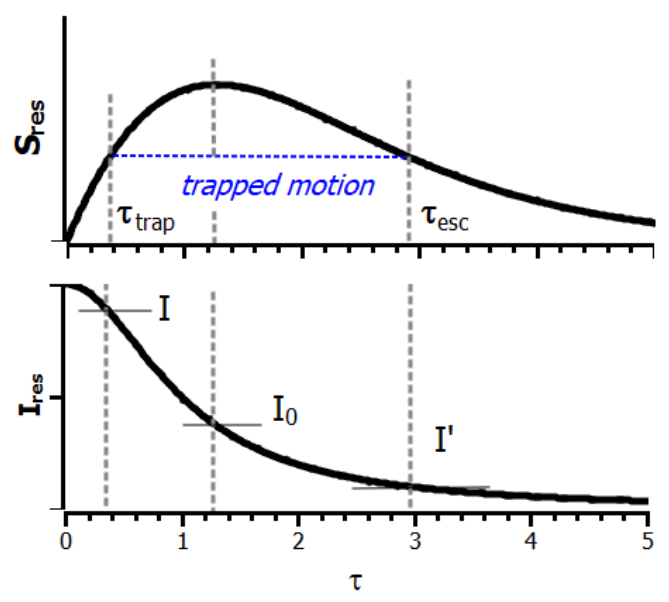

Figure 12: A schematic view of the area $S_{\text {res }}(\tau)$ and the resonant momentum $I_{\text {res }}(\tau)$. A particle is trapped at $\tau=\tau_{\text {trap }}$ when $S_{\text {res }}$ grows and $I=I_{\text {res }}$. During trapped motion, the momentum varies as $I_{\text {res }}(\tau)$. When, at $\tau=\tau_{\text {esc }}, S_{\text {res }}(\tau)$ returns back to $S_{\text {res }}\left(\tau_{\text {trap }}\right)$, the particle escapes from resonance. After the escape, the particle has a momentum $I_{\text {res }}\left(\tau_{\text {esc }}\right)=I^{\prime}$. For the sake of simplicity, we consider here systems with a single maximum of $S_{\text {res }}$ at $I=I_{0}$.

value at the moment of trapping $\tau_{\text {trap }}$, Fig. 12. During the trapped motion, the particle momentum $I$ varies following $I_{r e s}$, and the particles escape from resonance with larger (or smaller) values of $I$ (correspondingly with larger (or smaller) values of the energy $H(I, \tau)$ ). This effect corresponds to the particle acceleration by trapping. Since the duration of trapping can be rather long and variations of $I$ can be large (both are independent of $\varepsilon$ ), this process cannot be described as a local diffusion.

Transient particles passing through resonance without being trapped are scattered with a change of momentum $\Delta I$ given by Eq. (82). Two quantities describe the evolution of a particle distribution $\Psi(I)$ due to nonlinear and quasilinear scattering: the velocity of drift $V=\langle\Delta I\rangle / \tau_{0}$ and the diffusion coefficient $D=\left\langle(\Delta I)^{2}\right\rangle / 2 \tau_{0}$, where $\tau_{0}$ is the time between two successive passages through resonance. This expression for the diffusion coefficient $D$ coincides with the formula derived previously for a narrow wave spectrum in the quasi-linear theory [108]. Coefficients $\Pi(\tau), V(\tau)$, $D(\tau)$ can be rewritten as $\Pi(I), V(I), D(I)$ because $I=I_{\text {res }}(\tau)$ at resonance.

A trapping/escape event can be considered as a rapid change $I \rightarrow I^{\prime}$, where $I^{\prime}$ is defined by equations $I^{\prime}=I_{\text {res }}\left(\tau_{\text {esc }}\right)$, $S_{\text {res }}\left(\tau_{\text {trap }}\right)=S_{\text {res }}\left(\tau_{\text {esc }}\right)$, see Fig. 12. The functions $S_{\text {res }}(\tau)$ and $I_{\text {res }}(\tau)$ can be combined to get $S_{\text {res }}\left(I_{\text {res }}\right)=S_{\text {res }}(I)$ as $I=I_{\text {res }}$ at the resonance. Using $D, V$, and $\Pi / \tau_{0}$ we can write a Fokker-Planck equation describing the full evolution of the particle distribution function $\Psi(I)$ due to both scattering and trapping:

$$
\frac{\partial \Psi}{\partial t}=\frac{\partial}{\partial I}\left(D \frac{\partial \Psi}{\partial I}\right)-\frac{\partial(V \Psi)}{\partial I}+\int_{0}^{\infty}\left(\Psi(\tilde{I}) Q_{\tilde{I} \rightarrow I}-\Psi(I) Q_{I \rightarrow \tilde{I}}\right) d \tilde{I}
$$

Here $Q_{I^{\prime} \rightarrow I}$ is probability of a particle coming to $I$ via trapping, and $Q_{I \rightarrow I^{\prime}}$ is probability of a particle leaving from $I$. For $I>I_{0}$, the area $S_{\text {res }}$ grows and there is a finite $Q_{I \rightarrow I^{\prime}}=\left(\Pi(I) / \tau_{0}\right) \delta\left(w(I)-I^{\prime}\right)$. For $I<I_{0}$, the area $S_{\text {res }}$ decreases and particles escape from resonance: $Q_{I^{\prime} \rightarrow I}=\left(\Pi\left(I^{\prime}\right) / \tau_{0}\right) \delta\left(I-w\left(I^{\prime}\right)\right)$. In both cases, the input-output function $w\left(I^{\prime}\right)$ gives the value $I^{\prime}>I_{0}$ that particles should have when getting trapped to escape from resonance with $I<I_{0}$. The function $w$ is defined by the $S_{\text {res }}(I)$ profile, see Fig. 12. $\Pi\left(I^{\prime}\right)$ is the probability of particle trapping at $I^{\prime}>I_{0}$. The integral operator in Eq. (95) can then be expressed as $-\Psi(I) \Pi(I) / \tau_{0}$ for $I>I_{0}$ and $\Psi\left(I^{\prime}\right) \Pi\left(I^{\prime}\right)\left|d w / d I^{\prime}\right|^{-1} / \tau_{0}=(d V / d I) \Psi\left(I^{\prime}\right)$ for $I<I_{0}$, where we used

$$
\frac{d w\left(I^{\prime}\right)}{d I^{\prime}}=\frac{d I}{d I^{\prime}}=\frac{d V\left(I^{\prime}\right) / d I^{\prime}}{d V(I) / d I}=-\frac{\Pi\left(I^{\prime}\right) / \tau_{0}}{d V(I) / d I}
$$

and $V\left(I^{\prime}\right)=V(I)$ because $S_{\text {res }}\left(\tau_{\text {trap }}\right)=S_{\text {res }}\left(\tau_{\text {esc }}\right)$. Using these expressions and Eq. (92), we can rewrite Eq. (95) in its final form

$$
\frac{\partial \Psi}{\partial t}=\frac{\partial}{\partial I}\left(D \frac{\partial \Psi}{\partial I}\right)-V \frac{\partial \Psi}{\partial I}-\frac{d V}{d I}\left(\Psi-\Psi\left(I^{\prime}\right)\right) \Theta(I)
$$




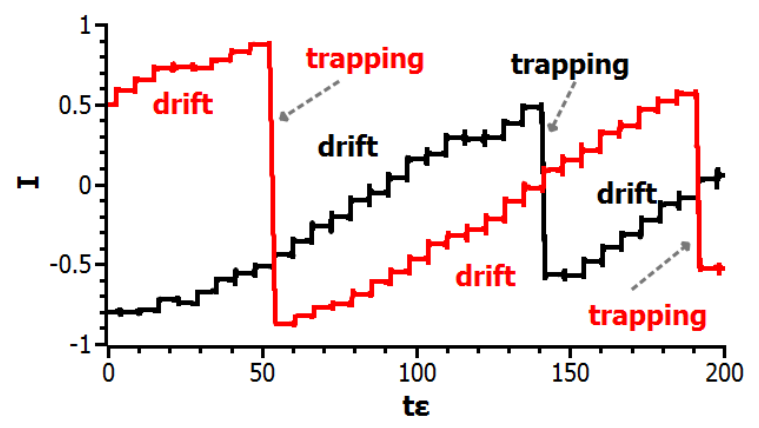

Figure 13: Two test trajectories (shown by black and red lines) for Hamiltonian (93).

where $I^{\prime}$ is defined by $w\left(I^{\prime}\right)=I$ and

$$
\Theta(I)= \begin{cases}1, & I<I_{0} \\ 0, & I \geq I_{0}\end{cases}
$$

Equation (96) is a Fokker-Planck equation generalized to include (1) the drift $V$ due to the mean change of the nonlinear scattering and (2) nonlocal term $\left(\sim \Psi(I)-\Psi\left(I^{\prime}\right)\right)$ corresponding to the fast transport in phase space due to trapping. The timescale of diffusion is $\varepsilon^{-1}$ ( since $D \sim \varepsilon$ ), whereas the timescales of drift and trapping-induced transport (determined by $V, d V / d I)$ are much shorter $\sim \varepsilon^{-1 / 2}$. For systems with small wave amplitudes $(a<1), V$ and $d V / d I$ vanish and Eq. (96) reduces to the usual quasi-linear diffusion equation

To check Eq. (96), we compared its solution with results of numerical integration of the initial system (93). We considered a simple example of Hamiltonian (93) with $\Lambda=0, g=1, I_{\text {res }}=\sin \tau$. The function $B(\tau)$ a periodic function of $\tau$ with a period $\tau_{0}=2 \pi / \varepsilon$. It has a single maximum in $\tau \in\left[\tau_{0} / 4,3 \tau_{0} / 4\right]$ and is zero for $\tau \in\left[0, \tau_{0} / 4\right]$, [3 $\left.\tau_{0} / 4, \tau_{0}\right]$. The resonant momentum $I_{R}$ varies from -1 to 1 and all particles pass through resonance over one period $\tau_{0}$. Figure 13 shows two test trajectories calculated over a long time interval. Particles are trapped at a positive $I$ and transported to negative $I$ (in this system $I_{0}=0$ ), whereas nonlinear drift $V$ transports particles back from negative $I$ to positive I. Both processes of trapping and drift coexist with the diffusive scattering. Particle behavior shown in Fig. 13 is a typical behavior in plasma systems with nonlinear wave-particle interactions.

For our test system, we calculate $\Pi, V$, diffusion coefficient $D$, and function $w(I)$, and express them as functions of $I=I_{R}$ (to calculate $D$ and $V$ we use the approximations from Fig. 11). To check the analytical formulas for $\Pi, V$, and $D$, we integrated numerically $10^{7}$ trajectories with different initial $I$ and calculated changes $\Delta I$ over a period $\tau_{0}$ that contained a single resonant interaction. The ratio of the number of particles which were trapped into resonance to the total number of particles gives the numerical probability of trapping $\Pi$. For untrapped particles, we calculate the average value of $\Delta I$ and its variance $\operatorname{Var}(\Delta I)$. These two functions, depending on the initial $I$, are then used to calculate the numerical values of $V$ and $D$. Figure 14 demonstrates that our analytical expressions quite accurate describe the trapping probability, drift velocity, and diffusion coefficient.

We substituted coefficients $V$ and $D$ into Eq. (96) and solved it numerically for a particular initial distribution $f_{0}(I)$. In Fig. 15, this solution is compared with direct numerical simulation of $10^{7}$ test particle trajectories for Hamiltonian (93). All expected effects of trapping and scattering are recovered: (1) fast transport of trapped particles from the initial distribution peak at positive $I$ toward negative $I$; (2) particle nonlinear drift toward positive $I$; and (3) a slower diffusive flattening. Figure 15 shows that Eq. (96) accurately describes all the major effects of nonlinear as well as quasi-linear wave-particle interactions.

Section summary. In this section we considered the evolution of particle distribution function due to resonant effects. For systems without the separatrix on the resonance plane, the energy drift is absent and we describe the effect energy diffusion. If the time between two resonance crossings does not depend on particle energy, the diffusive energy gain is limited. For systems with the separatrix, the energy drift due to trapping and nonlinear scattering dominates over the diffusion. Using the relation between trapping probability and scattering amplitude (see Eq. (92)), we derive generalized kinetic equation (96) including both trapping and nonlinear scattering effects. 

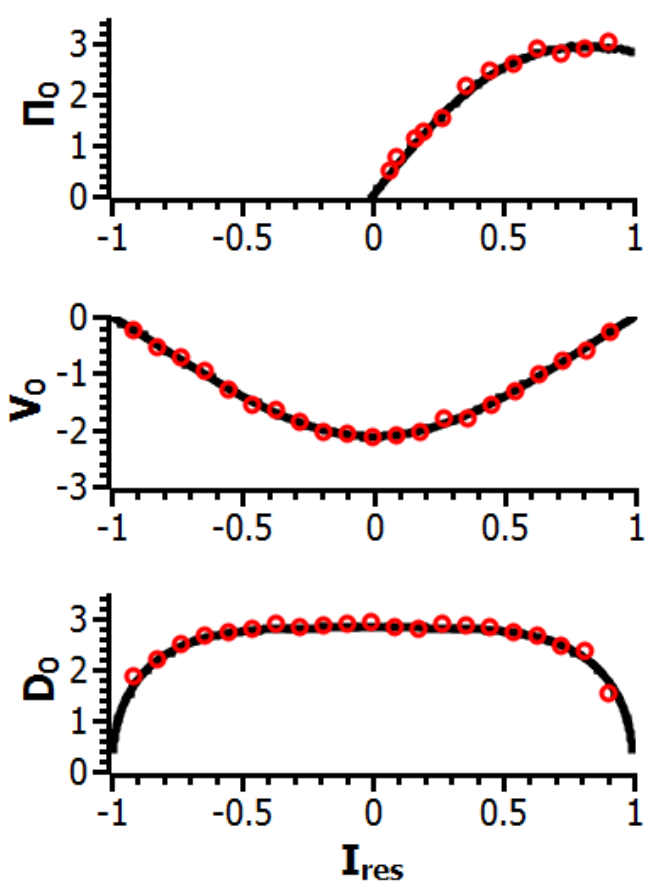

Figure 14: Analytical expressions (black curves) and numerical results (red circles) for $\Pi_{0}=\Pi \tau_{0} / \sqrt{\varepsilon}, V_{0}=V \tau_{0} / \sqrt{\varepsilon}$, and $D_{0}=D \tau_{0} / \varepsilon$.
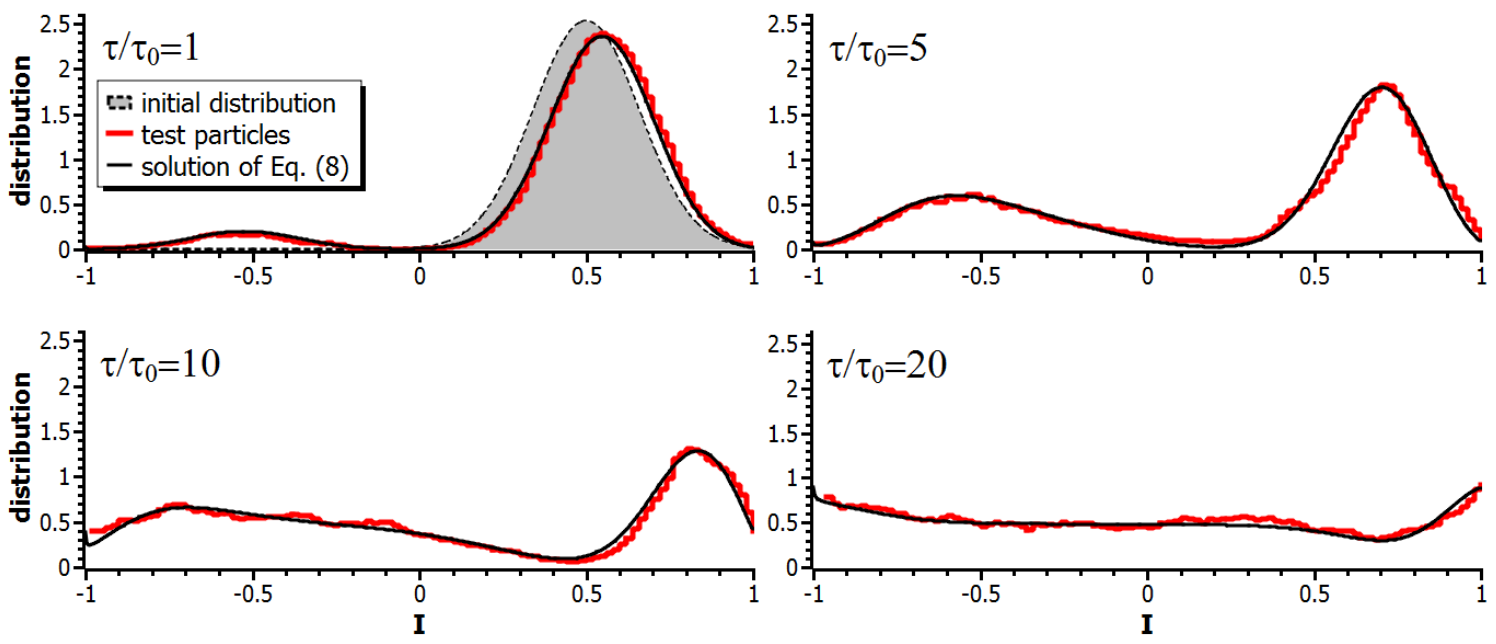

Figure 15: Particle distribution function $\Psi(I)$ at four moments of time. The initial distribution is displayed in grey, black curves show the numerical solution of generalized Fokker-Planck equation (96), and red curves are obtained from direct numerical integration of Hamiltonian system (93) with $10^{7}$ different initial conditions $(I, \phi)$. 


\section{Particle acceleration in nonrelativistic plasma systems}

In this section we consider several nonrelativistic plasma systems with particle trapping. We start with a simple system where an electrostatic wave propagates across a weak homogeneous magnetic field (Subsect. 6.1). In Subsect. 6.2 we describe a similar system, but instead of an electrostatic wave we consider a strong electromagnetic pulse (solitary wave). In Subsect. 6.3 we consider electrostatic wave resonating with charged particles in the systems with inhomogeneous background magnetic field.

Trapping is present when the electromagnetic field of the wave generates a force exceeding the Lorentz force of the background magnetic field. In this case the background magnetic field does not control particle motion and particles are effectively demagnetized. The Larmor (gyro-) rotation of particles is transformed into motion along the wave electric field and particles are accelerated.

All physical systems considered in this section can be described using a 1D or 2D configuration space. A background magnetic field $B_{0}$ is directed along the $z$-axis, particles move along $B_{0}$ and rotate around $B_{0}$ in the $(x, y)$ plane (see Fig. 16). In the case of an inhomogeneous magnetic field $B_{0}(z)$, the Maxwell equations require the presence of an additional magnetic field component $B_{0 x}=-d\left(\int B_{0}(z, \tilde{x}) d \tilde{x}\right) / d z$ directed along the $x$-axis [112]. The corresponding vector potential still has a single component $\mathbf{A}=A_{y} \mathbf{e}_{y}=\left(\int B_{0}(z, \tilde{x}) d \tilde{x}\right) \mathbf{e}_{y}$. An external inductive electric field $E_{y}$ can be included in the Hamiltonian as an additional term $-c \int E_{y}(t) d t$ in $A_{y}$, whereas an external electrostatic field $E_{z}, E_{x}$ can be described by a scalar potential $\Phi(x, z, t)$. Hamiltonian of a particle with a charge $e$ and mass $m$ is

$$
H=\frac{1}{2 m}\left(p_{x}^{2}+p_{z}^{2}\right)+\frac{1}{2 m}\left(p_{y}-\frac{e}{c} A_{y}(x, z, t)\right)^{2}+e \Phi(x, z, t)
$$

Hamiltonian (97) does not depend on $y$, and this condition is satisfied in the perturbed system (in the presence of waves) as well. Thus, $p_{y}=$ const and we can set $p_{y}=0$ via time shift (in case of $E_{y} \neq 0$ ) or via shift of the origin of $x$ axis. The Hamiltonian becomes

$$
H=\frac{1}{2 m}\left(p_{x}^{2}+p_{z}^{2}\right)+\frac{1}{2 m}\left(\frac{e}{c} A_{y}(x, z, t)\right)^{2}+e \Phi(x, z, t)
$$

The corresponding Hamiltonian equations are

$$
\begin{aligned}
\dot{x} & =\frac{\partial H}{\partial p_{x}}=\frac{1}{m} p_{x}, \quad \dot{z}=\frac{\partial H}{\partial p_{z}}=\frac{1}{m} p_{z} \\
\dot{p}_{x} & =-\frac{\partial H}{\partial x}=\frac{e^{2}}{m c^{2}} B_{0} A_{y}-e \frac{\partial \Phi}{\partial x} \\
\dot{p}_{z} & =-\frac{\partial H}{\partial z}=-\frac{e^{2}}{m c^{2}} B_{0 x} A_{y}-e \frac{\partial \Phi}{\partial z}
\end{aligned}
$$

In case of $B_{0}=$ const, $E_{y}=0, \Phi=0$ we obtain $B_{0 x}=0$, and the motion along $z$ is decoupled from the motion in the $(x, y)$ plane: $p_{z}=$ const. Thus, Eqs. (99) can be rewritten as

$$
m \dot{x}=p_{x}, \quad \dot{p}_{x}=m \Omega_{0}^{2} x
$$

where $A_{y}=x B_{0}$, and $\Omega_{0}=e B_{0} / m c$ is the Larmor (gyro-) frequency of particle oscillations around $B_{0}$. These oscillations are those of a linear pendulum.

\subsection{Electrostatic wave propagating across a weak magnetic field}

This subsection discusses a system where particles can be trapped by an electrostatic wave propagating across a sufficiently weak background magnetic field. Weakness of the magnetic field guarantees that gyrorotation is slow enough to consider wave phase as a fast variable. In this case, particles interact with waves through the Cherenkov resonance $\mathbf{k} \cdot \mathbf{v}=\omega$ where $\mathbf{v}$ is a particle velocity and $\mathbf{k}$ is a wavevector. Such a configuration of a background magnetic field and a wave field is a classical problem of plasma physics: the Landau damping in presence of a weak magnetic field $[42,113,114]$. Some generalisation of results presented in this subsection can be found in $[115,116]$. 


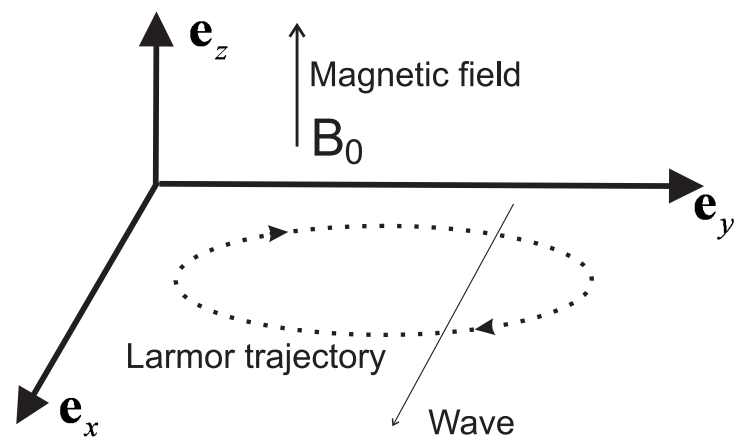

Figure 16: Schematic view of the system configuration.

Consider a nonrelativistic electron moving in a background uniform magnetic field $B_{0}$ and a wave electrostatic field. The wave phase is $\phi=\phi_{0}+k x-\omega t$, where $\omega \gg \Omega_{0}=e B_{0} / m c$ and the wave phase velocity $v_{\phi}=\omega / k=c o n s t$ is similar to the particle velocity. The corresponding equations of motion are (see Eqs. (100))

$$
m \dot{x}=p_{x}, \quad \dot{p}_{x}=m \Omega_{0}^{2} x-e k \Phi_{0} \cos \phi
$$

where $\Phi_{0}$ is the amplitude of the scalar potential. Particles may experience both scattering and trapping. The left panel in Fig. 17 shows particle trajectory without trapping. The particle moves around a closed trajectory in the $\left(x, p_{x}\right)$ plane and crosses the resonance $p_{x}=m v_{\phi}$ twice on one period. At each crossing there is a change of momentum $p_{x}$ and energy $h=p_{x}^{2} / 2+\Omega_{0}^{2} x^{2} / 2$. When $\Phi_{0}$ is large enough, this change has nonzero mean and a dispersion (see Sect. 4). At two consecutive crossings the changes of energy $h$ have nonzero average values with the opposite signs, and these changes approximately compensate each other (see Fig. 17, left panel). If trapped, a particle leaves a closed trajectory in the $\left(x, p_{x}\right)$ plane and starts moving along the resonant trajectory $p_{x}=m v_{\phi}$ (see Fig. 17, right panel). Trapping occurs at a negative $x$ and trapped particles move with a positive $p_{x}$. After trapping particle energy starts decreasing (see description in Sect. 3). When the trapped particle reaches $x \sim 0$, the energy $h$ and the $x$ coordinate start increasing and $h$ returns to the initial value when $x$ reaches $-x_{\text {trap }}$. At this position, the particle escapes from resonance and starts moving along a new closed trajectory in the $\left(x, p_{x}\right)$ plane (see Fig. 17, right panel).

To consider particle motion near the resonance, we rewrite system (101) in terms of the phase $\phi$ :

$$
\frac{\ddot{\phi}}{k}=\Omega_{0}^{2} x-\frac{e k \Phi_{0}}{m} \cos \phi
$$

As $\omega \gg \Omega_{0}$, the phase $\phi$ changes significantly faster than the variable $x$. Thus we can consider $x$ as a slowly changing parameter and Eq. (102) corresponds to the Hamiltonian:

$$
H_{\phi}=\frac{1}{2} k^{2} P_{\phi}^{2}-\Omega_{0}^{2} k^{-1} x \phi+\frac{e \Phi_{0}}{m} \sin \phi
$$

where $P_{\phi}=\dot{\phi} / k^{2}$ is a momentum conjugate to $\phi$. Hamiltonian (103) coincides with the Hamiltonian (34) of a nonlinear pendulum with torque. The area $S_{\text {res }}$ is shown in the left panel in Fig. 7, and is given by Eq. (42):

$$
S_{\text {res }}=\frac{2 \Omega_{0}}{k^{3 / 2}} \sqrt{2|x|} f_{s}(a), \quad a=\frac{e k \Phi_{0}}{m \Omega_{0}^{2}|x|}
$$

When $x<0$, the area $S_{\text {res }}$ grows as $(-x)$ decreases, see Fig. 17, and particle becomes trapped at $x_{\text {trap }}<0$. The trapped particle moves from negative to positive $x$. When it reaches the coordinate $x_{\text {res }}=-x_{\text {trap }}$, the area $S_{\text {res }}$ becomes equal to its value at the moment of trapping, and the trapped particle escapes from resonance. As a result of such trappingescape event, the particle energy does not change more than by the value defined as a resonance width (63):

$$
\Delta h=\frac{p_{x}}{2 m}\left|\left\langle\Delta p_{x}\right\rangle\right|=\sqrt{\frac{1}{2 k\left|x_{\text {trap }}\right|}} \frac{e k v_{\phi} \Phi_{0}}{\Omega_{0}}\left|f_{p}\right|=e \Phi_{0} \frac{\omega}{\Omega_{0}} \sqrt{\frac{1}{2 k\left|x_{\text {trap }}\right|}}\left|f_{p}\right|
$$



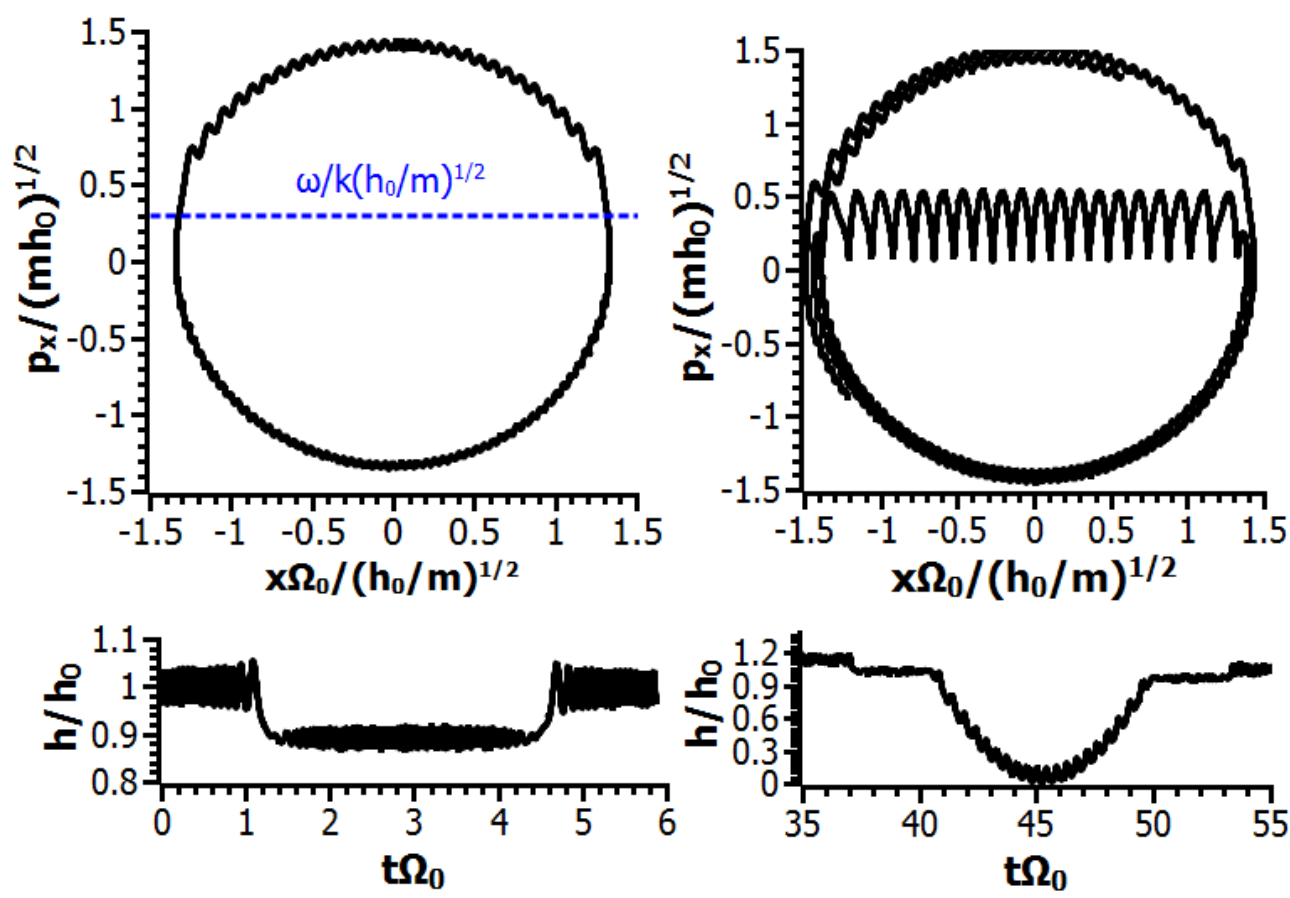

Figure 17: Two particle trajectories of system (101): scattering (left panels) and trapping (right panels) are shown. System parameters are: $\omega / k \sqrt{h_{0} / m}=0.3, \omega / \Omega_{0}=100$, initial energy $h_{0}=3(\omega / k)^{2} m$.

If the wave amplitude is large enough ( $a \gg 1$ in Eq. (104)), then $f_{p} \approx 2 f_{s} / a \approx 8 \sqrt{2 / a}$ and Eq. (105) takes the form:

$$
\Delta h=8 \sqrt{e \Phi_{0} v_{\phi}^{2} m}
$$

Therefore, the resonance width (and a range of energy variation) is proportional to square root of wave amplitude and the energy of a resonant particle $[117,118]$.

In terms of the energy evolution, scattering of particles on resonance can be considered as a combination of random changes with zero mean (diffusion) and some mean energy change (drift in energy space, see Eq. (64)). The the sign of momentum and energy changes is opposite at $x_{\text {trap }}$ and $x_{e s c}=-x_{\text {trap }}$. As a result, on one period of the Larmor rotation, particles change their energy by values distributed around zero (see Fig. 17). This scattering results in diffusion in energy space (see a detailed description in Sect. 5). Significant and fast energy change in such a system is possible only if an external force breaks the symmetry of trapping and escape coordinates making $x_{e s c}$ no longer equal to $-x_{\text {trap }}$. In this case, the difference of energies at the moment of trapping and escape is not zero [115].

\subsection{Front of plasma injection}

As shown in the previous subsection, trapping into resonance with an electrostatic wave does not result in the particle acceleration. A situation is different for electromagnetic waves [119, 120]. An interesting example of the system with an electromagnetic wave has been recently found in the Earth magnetosphere, where transient reconnection of magnetic field lines runs strong plasma flows [121, 122]. Propagating across a weak magnetic field, these flows generate strong electromagnetic perturbations localized near the leading edge of flows. Modern spacecraft observations demonstrate that plasma flows with characteristics configuration of electromagnetic fields are commonly detected in different planetary magnetospheres [123, 124, 125] and solar corona [see references in 126]. The leading front of such plasma flows represents the electromagnetic pulse (a strong electromagnetic soliton wave). Charged particles can be trapped into the Cherenkov resonance and accelerated by such a pulse [127, 128]. 

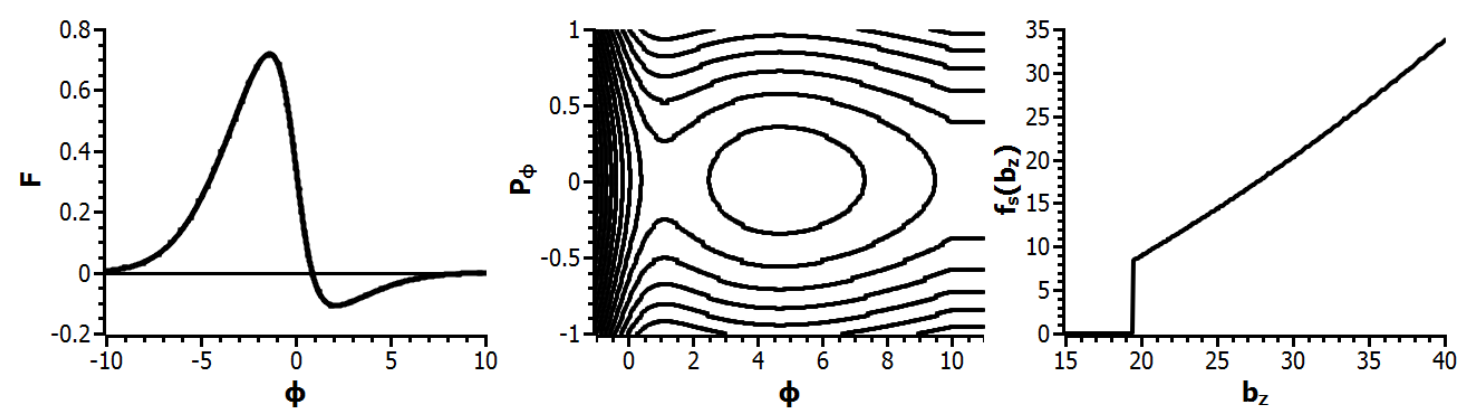

Figure 18: Function $F(\phi)$ (left panel), phase portrait of Hamiltonian $H_{\phi}$, Eq. (111) (central panel), function $\tilde{f}_{s}(a)$ Eq. (112) (right panel).

A simple model of a plasma flow front describes a magnetic field directed along the $z$-axis and propagating with a constant velocity $v_{\phi}$ along the $x$-axis: $B_{z, f}=\delta B_{z} f(\phi)$, where $\delta B_{z}>B_{0}$ is an amplitude of the front magnetic field, and $f(\phi)$ defines the distribution of the front field along the $x$-axis [127]. Several more general configurations were considered in $[129,130]$. Phase $\phi=\phi_{0}+\left(x-v_{\phi} t\right) / L$ depends on the front scale $L$, which is usually much smaller than the gyroradius of hot ions observed in planetary magnetospheres. The front electric field is equal to $E_{y, f}=\left(v_{\phi} / c\right) B_{z, f}$. The corresponding vector potential is $A_{y, f}=\delta B_{z} L \int f(\phi) d \phi=\delta B_{z} L F(\phi)$. An example of $F(\phi)$ profile is shown in Fig. 18(a). Hamiltonian (98) of an ion moving in such an electromagnetic field is

$$
H=\frac{1}{2 m} p_{x}^{2}+\frac{1}{2 m} \frac{e^{2}}{c^{2}}\left(B_{0} x+\delta B_{z} L F(\phi)\right)^{2}
$$

where we set $p_{z}=0$, because the Hamiltonian is independent of $z$. The Hamiltonian equations are

$$
m \dot{x}=p_{x}, \quad \dot{p}_{x}=-\frac{1}{m} \frac{e^{2}}{c^{2}}\left(B_{0}+\delta B_{z} f(\phi)\right)\left(B_{0} x+\delta B_{z} L F(\phi)\right)
$$

Due to smallness of $L$, we can omit the term $\sim L$ and rewrite Eqs. (108) as

$$
m \dot{x}=p_{x}, \quad \dot{p}_{x}=-m \Omega_{0}^{2} x\left(1+b_{z} f(\phi)\right)
$$

where $b_{z}=\delta B_{z} / B_{0}$. The equation for phase $\phi$ in the vicinity of the resonance $\dot{\phi}=0$ can be written as

$$
\frac{\ddot{\phi}}{k}=-\Omega_{0}^{2} x\left(1+b_{z} f(\phi)\right)
$$

Due to the fast variation of phase $\phi$, we can assume $x \approx$ const and obtain a Hamiltonian for Eq. (110):

$$
H_{\phi}=\frac{1}{2} k^{2} P_{\phi}^{2}+\Omega_{0}^{2} k^{-1} x\left(\phi+b_{z} F(\phi)\right)
$$

where $P_{\phi}=\dot{\phi} / k^{2}$ is a momentum conjugate to $\phi$. Hamiltonian (111) is similar to Hamiltonian (34) for system (5) when the amplitude of perturbation and the torque are proportional to slowly changing variable $x$. The phase portrait of Hamiltonian (111) is shown in Fig. 18 (central panel). Although the perturbation $\sim F(\phi)$ is not periodic, within a certain range of $\phi$ the phase portrait is similar to one shown in Fig. 4.

For Hamiltonian (111), the area surrounded by the separatrix can be written using Eqs. (37-39):

$$
\begin{array}{r}
S_{r e s}=\frac{2 \Omega_{0}}{k^{3 / 2}} \sqrt{2|x|} \tilde{f}_{s}(a), \quad a=b_{z} \\
\tilde{f}_{S}(a)=\int_{\phi_{X}}^{\phi_{\max }} \sqrt{\left(\phi_{X}-\phi\right)+a\left(F\left(\phi_{X}\right)-F(\phi)\right)} d \phi
\end{array}
$$



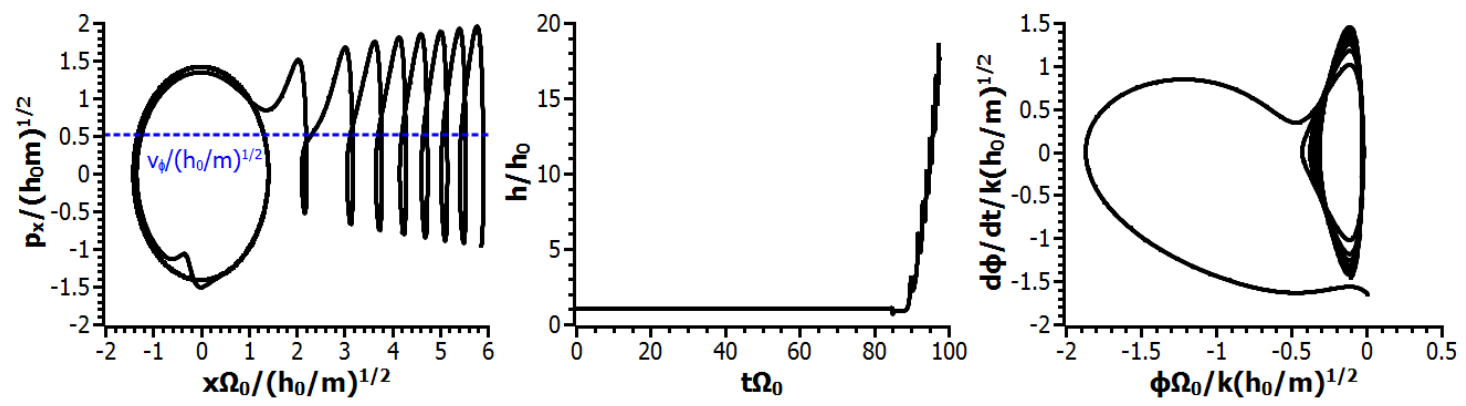

Figure 19: Numerical integration of system (109). Trajectory in the ( $\left.x, p_{x}\right)$ plane (left panel), particle energy as a function of time (central panel), and trajectory in $(\phi, \dot{\phi})$ (right panel). Parameters are $\omega / \Omega_{0}=100, b_{z}=20, \omega / k \sqrt{h_{0} / m}=0.5$, initial energy $h_{0}=3(\omega / k)^{2} m$.

Function $\tilde{f}_{s}(a)$ in (112) is independent of $x$ and depends only on the amplitude of perturbation $b_{z}$ (see Fig. 18(right panel)). For $b_{z}>1$, we have $\tilde{f}_{s} \neq 0$ and, thus, $S_{\text {res }}$ increases with $x$. Once trapped, particles cannot leave the region of oscillations around the resonance.

An example of such a trajectory is shown in Fig. 19. The particle rotates around the background magnetic field (closed trajectories in the $\left(x, p_{x}\right)$ plane) and crosses the resonance curve $p_{x}=m v_{\phi}$ twice on each period of rotation. In contrast with the system shown in Fig. 17, for system (107) the area $S_{\text {res }}$ increases in resonance for positive $x$. Thus trapping happens at the right-hand intersection of trajectory by the resonance curve (see explanation of $S_{\text {res }}$ behaviour in Sect. 3). As a result, trapped particles start moving with growing $x$ (compare Figs. 17 and 19). Energy of a trapped particle increases as $\sim t^{2}$ (see Eq. (54)). Trapped oscillations have a decreasing amplitude of $\phi$ oscillations and increasing amplitude of $\dot{\phi}$ oscillations (see Fig. 19 (right panel) and [109, 131]).

Different profiles of $f(\phi)$ may appear in concrete models describing particle trapping and acceleration by electromagnetic waves or pulses: $f(\phi)=\sin \phi[119,109], f(\phi)=\tanh (\phi)[120,132], f(\phi)=\phi \exp \left(-\left(\phi+\phi_{0}\right)^{2}\right)$ [127]. Regardless of a specific type of $f(\phi)$, trapping into resonance with electromagnetic (as opposite to electrostatic) "wave" results in an unlimited acceleration, as the area $S_{\text {res }}$ given by Eq. (112) always increases and trapped particles cannot escape from resonance without external perturbations of system (see Sect. 8).

\subsection{Nonlinear electrostatic localized structures in inhomogeneous magnetic field}

In planetary aurora regions and Earth radiation belts, strong electron beams generate various electrostatic solitary waves, such as double layers or electron holes propagating along a background magnetic field. In an inhomogeneous magnetic field, such structures can trap particles into the Landau resonance and accelerate them to high energies $[133,134]$.

Hamiltonian of electron in a strong inhomogeneous magnetic field and an electrostatic solitary wave field can be written as (see Eq. (98))

$$
H=\frac{1}{2 m}\left(p_{x}^{2}+p_{z}^{2}\right)+\frac{1}{2 m}\left(\frac{e}{c} B_{0}(z) x\right)^{2}-e \Phi_{0} F(\phi)
$$

where $F(\phi)$ defines the shape of a scalar potential (see Fig. 20)(left panel)), and $\Phi_{0}$ is an amplitude of a scalar potential. For the sake of simplicity, we assume that wave phase velocity $v_{\phi}$ is constant:

$$
\phi=\left(z-v_{\phi} t\right) / L
$$

The spatial scale of a solitary wave $L$ is much smaller than a scale of magnetic field inhomogeneity $B_{0}(z)$.

In the absence of perturbations, $\Phi_{0} \sim 0$, Hamiltonian equations for Hamiltonian (113) are

$$
\begin{aligned}
\dot{x} & =p_{x} / m, \quad \dot{z}=p_{z} / m \\
\dot{p}_{x} & =-m \Omega_{0}^{2}(z) x \\
\dot{p}_{z} & =-m \Omega_{0}(z) \Omega_{0}^{\prime} x^{2}
\end{aligned}
$$



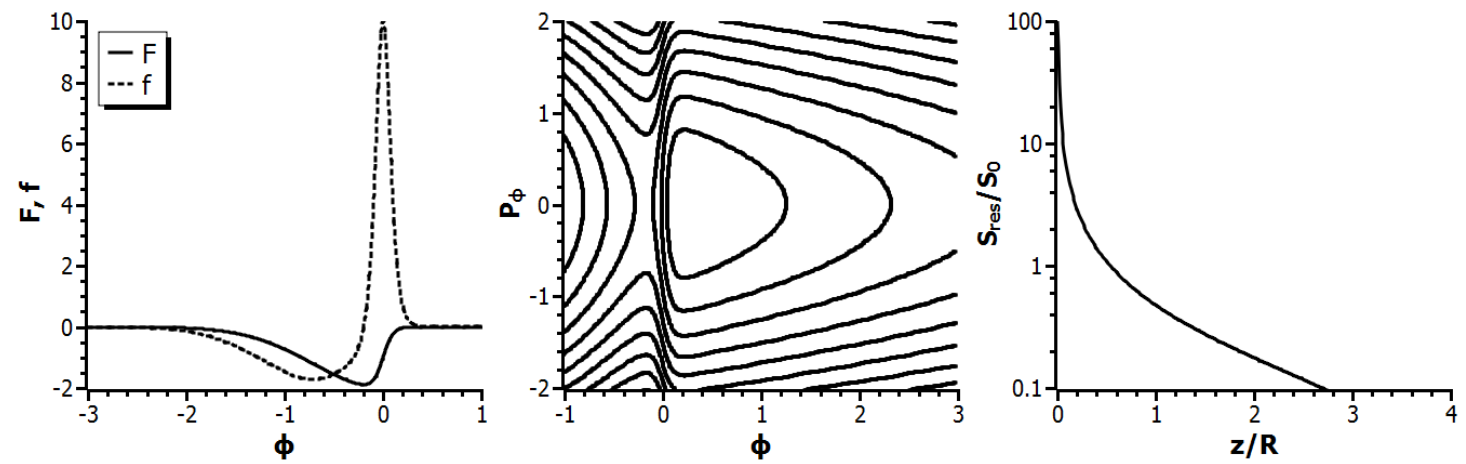

Figure 20: Resonance interaction of particles with electrostatic localized structures. Profiles $F(\phi)$ and $f=d F / d \phi$ (left panel). Phase portrait of (121) (central panel). The area $S_{\text {res }}$ given by Eq. (122) (right panel) with $S_{0}=2 \sqrt{4 I_{x} L^{3} / R m}$ and $e \Phi_{0} R / 2 I_{x} L=0.5$.

where $\Omega_{0}(z)=e B_{0}(z) / m c$ and $\Omega_{0}^{\prime}=\partial \Omega_{0} / \partial z$. In a strong background magnetic field the gyrofrequency $\Omega_{0}$ is large enough to make oscillations in the $\left(x, p_{x}\right)$ plane significantly faster than motion in the $\left(z, p_{z}\right)$ plane. For these fast oscillations we can introduce new conjugate variables $I_{x}, \theta$ where $I_{x}$ is an adiabatic invariant (see more details in Appendix B):

$$
I_{x}=\frac{1}{2 \pi} \oint \sqrt{2 m H-p_{z}^{2}-\left(m \Omega_{0}(z) x\right)^{2}} d x=\frac{2 m H-p_{z}^{2}}{2 m \Omega_{0}(z)}
$$

The new Hamiltonian does not depend on $\theta$, therefore $I_{x}$ is a constant:

$$
H=\frac{1}{2 m} p_{z}^{2}+I_{x} \Omega_{0}(z)
$$

The perturbed Hamiltonian we can be written as

$$
H=\frac{1}{2 m} p_{z}^{2}+I_{x} \Omega_{0}(z)-e \Phi_{0} F(\phi)
$$

There is an effective potential energy $I_{x} \Omega_{0}(z)$ in Hamiltonian (118). Particle motion in the $\left(z, p_{z}\right)$ plane is described by equations

$$
\dot{z}=p_{z} / m, \quad \dot{p}_{z}=-I_{x} \Omega_{0}^{\prime}(z)+\frac{e \Phi_{0}}{L} F^{\prime}(\phi)
$$

Using definition of phase (114) we can rewrite Eqs. (119) as

$$
L^{2} \ddot{\phi}=-\frac{I_{x} L}{m} \Omega_{0}^{\prime}(z)+\frac{e \Phi_{0}}{m} F^{\prime}(\phi)
$$

As the phase $\phi$ changes much faster than $z$, we can integrate Eq. (120) to obtain Hamiltonian

$$
H_{\phi}=\frac{1}{2 L^{2}} P_{\phi}^{2}+\frac{I_{x} L}{m} \Omega_{0}^{\prime}(z) \phi-\frac{e \Phi_{0}}{m} F(\phi)
$$

where $P_{\phi}=\dot{\phi} L^{2}$ is a momentum conjugate to $\phi$. In Hamiltonian (121) torque $\sim \Omega_{0}^{\prime}$ is produced by the inhomogeneity of the background magnetic field. Phase portrait of Hamiltonian (121) is shown in Fig. 20(central panel). For a large enough potential $\left(e \Phi_{0}>I_{x} L \Omega_{0}^{\prime}\right)$, there is a region filled with closed trajectories in the phase plane. Area of this region is defined by Eq. (40):

$$
S_{\text {res }}=2 \sqrt{2\left|\Omega_{0}^{\prime}\right| \frac{I_{x} L^{3}}{m}} f_{s}(a), \quad a=e \Phi_{0} / I_{x} L \Omega_{0}^{\prime}
$$

Figure 20(right panel) shows that $S_{\text {res }}$ has a maximum at $z=0$ (where $\Omega_{0}^{\prime}=0$ ) and decrease with $|z|$. Thus, particles can be trapped by solitary wave at large $-z_{\text {trap }}$ where $S_{\text {res }}$ grows along the resonant trajectory $\dot{z}=v_{\phi}>0$ and they 

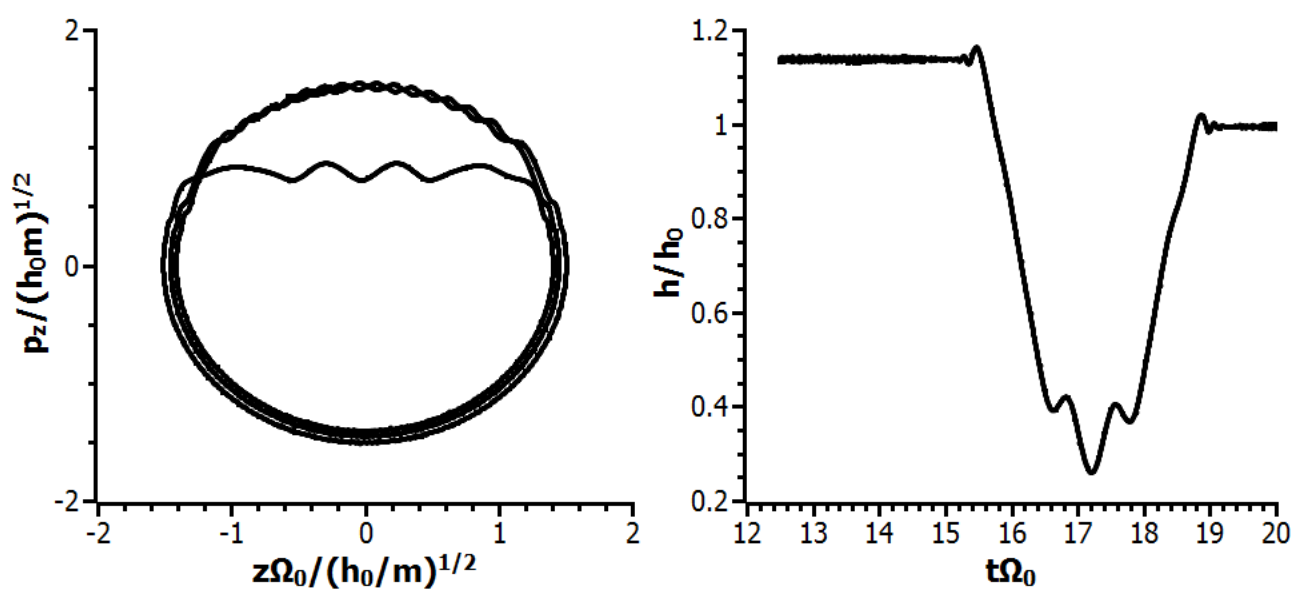

Figure 21: A trajectory and the corresponding energy obtained with numerical integration of (120). Variables are normalised on $\Omega_{0}(0)$ and initial energy $h_{0}$. System parameters are: $v_{\phi} / \sqrt{h_{0} / m}=0.8, e \Phi_{0} R / L I_{x} \Omega_{0}(0)=1, R \Omega_{0}(0) / L=100, R \Omega_{0}(0) / \sqrt{h_{0} / m}=\sqrt{2}$. To calculate these trajectories, we chose a simple model of background magnetic field $B_{0} \approx\left(1+(z / R)^{2}\right)$.

escape from resonance at $z_{e s c}=-z_{\text {trap }}$. Corresponding change of particle energy in the resonance is described by Eq. (117) with $p_{z}=m v_{\phi}$ and $z=z_{\text {trap }}+v_{\phi} t$ :

$$
h=\frac{1}{2} m v_{\phi}^{2}+I_{x} \Omega_{0}\left(z_{\text {trap }}+v_{\phi} t\right)
$$

The resonant energy decreases for $z_{\text {trap }}+v_{\phi} t<0$ and increases for $z_{\text {trap }}+v_{\phi} t>0$. Due to the symmetry with respect to the $z=0$ plane, there is no net energy gain for a trapping-escape event, like in the system in Subsect. 6.1.

A typical example of particle trajectory is shown in Fig. 21. The particle moves along a closed trajectory in the $\left(z, p_{z}\right)$ plane and becomes trapped with negative $z_{\text {trap }}$. The trapped motion takes the particle from $z=z_{\text {trap }}$ to $z=z_{\text {esc }} \approx-z_{\text {trap }}>0$ almost horizontally in Fig. 21(left panel). Before the particle cross the $z=0$ plane, the particle energy decreases, whereas for $z>0$ the particle energy increases and reaches approximately the same value as at the moment of trapping.

In realistic space plasma systems such as Earth radiation belts, solitary electrostatic structures are often generated around local minima of magnetic field, defined by $\Omega_{0}^{\prime}=0$. The wave amplitude $\Phi_{0}$ depends on $z$ and increases with $|z|$. This condition allows solitary waves to trap and accelerate electrons $[135,136]$.

\subsection{Obliquely propagating electromagnetic waves}

The obliquity of waves (the presence of a wave number component directed along the background magnetic field) affects the trapped particle motion and leads to particle escape from resonance [106, 137]. For the system from Subsect. 6.2 with a periodic wave $F(\phi)=\sin \phi$ and $\phi=k_{x} x+k_{z} z-\omega t$, Hamiltonian (107) is

$$
H=\frac{1}{2 m}\left(p_{x}^{2}+p_{z}^{2}\right)+\frac{1}{2 m} \frac{e^{2}}{c^{2}}\left(B_{0} x+\delta B_{z} k^{-1} \sin \phi\right)^{2}
$$

where $k=\sqrt{k_{x}^{2}+k_{z}^{2}}$. System (124) contains two pairs of conjugate variables $\left(x, p_{x}\right),\left(z, p_{z}\right)$ and is more complicated than the original system with $k_{z}=0$. Assuming that the wave amplitude $\delta B_{z}=$ const is much smaller than $B_{0}$, we expand Hamiltonian (124):

$$
H=\frac{1}{2 m}\left(p_{x}^{2}+p_{z}^{2}\right)+\frac{1}{2} m \Omega_{0}^{2} x^{2}+m \Omega_{0}^{2} \frac{x}{k} b_{z} \sin \phi
$$

where $\Omega_{0}=e B_{0} / m c=$ const, $b_{z}=\delta B_{z} / B_{0}$. We introduce $\phi$ as a new canonical variable using a generating function

$$
R=I\left(k_{x} x+k_{z} z-\omega t\right)+P_{x} x+P_{z} z
$$


where $P_{x}, P_{z}$ are new momenta, whereas $x$ and $z$ remain unchanged. The new Hamiltonian does not depend on $z$, thus we can set $P_{z}=$ const $=0$ to get

$$
H=-\omega I+\frac{1}{2 m}\left(\left(P_{x}+k_{x} I\right)^{2}+\left(k_{z} I\right)^{2}\right)+\frac{1}{2} m \Omega_{0}^{2} x^{2}+m \Omega_{0}^{2} \frac{x}{k} b_{z} \sin \phi
$$

The resonance condition $\dot{\phi}=0$ can be written as

$$
\frac{\partial H}{\partial I}=-\omega+\frac{1}{m}\left(k_{x}\left(P_{x}+k_{x} I\right)+k_{z}^{2} I\right)=0
$$

with the solution

$$
I_{\text {res }}=\frac{m k v_{\phi}-k_{x} P_{x}}{k^{2}}
$$

where $v_{\phi}=\omega / k=$ const. We expand Hamiltonian (127) around $I-I_{\text {res }}$ :

$$
\begin{aligned}
H & =\Lambda+\frac{1}{2 m} k^{2}\left(I-I_{\text {res }}\right)^{2}+m \Omega_{0}^{2} \frac{x}{k} b_{z} \sin \phi \\
\Lambda & =-\omega I_{\text {res }}+\frac{1}{2 m}\left(\left(P_{x}+k_{x} I_{\text {res }}\right)^{2}+\left(k_{z} I_{\text {res }}\right)^{2}\right)+\frac{1}{2} m \Omega_{0}^{2} x^{2} \\
& =-\frac{1}{2} m v_{\phi}^{2}+\frac{1}{2 m} P_{x}\left(\frac{k_{z}^{2}}{k^{2}} P_{x}+2 m v_{\phi} \frac{k_{x}}{k}\right)+\frac{1}{2} m \Omega_{0}^{2} x^{2}
\end{aligned}
$$

We introduce new variable $P_{\phi}=I-I_{\text {res }}$ and write $H-\Lambda$ in the form of Hamiltonian (34)

$$
H_{\phi}=\frac{1}{2 m} k^{2} P_{\phi}^{2}+m \Omega_{0}^{2} \frac{k_{x}}{k^{2}} x \phi+m \Omega_{0}^{2} \frac{x}{k} b_{z} \sin \phi
$$

where $x$ is a slow variable. It follows from Eq. (40) for $b_{z}>k_{x} / k$ there is a surrounded by the separatrix region with trapped particles. Its area is

$$
S_{\text {res }}=2 \frac{m \Omega_{0}}{k^{2}} \sqrt{2 k_{x}|x|} f_{s}(a), \quad a=b_{z} k / k_{x}
$$

When $S_{\text {res }} \sim \sqrt{|x|}$ grows in the process of motion, particles can be trapped into resonance. In the $\left(x, p_{x}, p_{z}\right)$ space, the isoenergetic surface of $H=h=$ const is

$$
h=\frac{1}{2 m}\left(p_{x}^{2}+p_{z}^{2}+m^{2} \Omega_{0}^{2} x^{2}-\left(k m v_{\phi} / k_{z}\right)^{2}\right)
$$

where $p_{x}=P_{x}+k_{x} I$ and $p_{z}=k_{z} I-k m v_{\phi} / k_{z}$. This is an ellipsoid centered at the origin $\left(x=0, p_{x}=0, p_{z}=0\right)$. To obtain equation for the resonant plane, we combine Eq. (129) and $p_{z}=k_{z} I-k m v_{\phi} / k_{z}$ :

$$
\frac{k_{x}}{k} p_{x}+\frac{k_{z}}{k} p_{z}=0
$$

The intersection of these two surfaces is a resonant curve $\mathcal{R}$. The Larmor rotation in the background magnetic field resides on intersections of the isoenergetic surface and planes $p_{z}=$ const. We use notation $\mathcal{L}_{\alpha}$ for the intersection of the isoenergetic sphere and a plane $p_{z}=p_{z}^{(\alpha)}=$ const.

Characteristic particle motion is shown in Fig. 22. Let initially $p_{z}=p_{z}^{(*)}<0$ and let $\mathcal{L}_{*}$ cross the resonant curve. In the process of Larmor motion the particle repeatedly passes through the resonance. Eventually, at a certain $p_{z}=p_{z}^{(0)}$ close to $p_{z}^{(*)}$, it is trapped into resonance. Of the two points where $\mathcal{R}$ crosses $\mathcal{L}_{0}$, trapping occurs where $S_{\text {res }}$ grows, i.e., where $d|x| / d t>0$. Direction of trapped motion on $\mathcal{R}$ is determined by Hamiltonian $\Lambda$ (Fig. 22(left panel)). The trapped particle moves along $\mathcal{R}$ until $S_{\text {res }}$ returns to its value at the moment of trapping. Upon that return, particle escapes from resonance. It follows from the symmetry with respect to the origin that after the escape the particle moves along another Larmor trajectory $\mathcal{L}_{1}$ with $p_{z}=p_{z}^{(1)}=2 m v_{\phi} k / k_{z}-p_{z}^{(0)}$. Projections of $\mathcal{L}_{0}$ and $\mathcal{L}_{1}$ on the $\left(x, p_{x}\right)$ plane coincide. Hence net change of the particle energy $E=\left(p_{x}^{2}+\Omega_{0}^{2} m^{2} x^{2}+p_{z}^{2}\right) / 2 m$ due to trapping into resonance is

$$
\Delta E=\frac{1}{2 m} \Delta\left(p_{z}^{2}\right)=2 v_{\phi}\left(m v_{\phi}-p_{z}^{(0)}\right)
$$



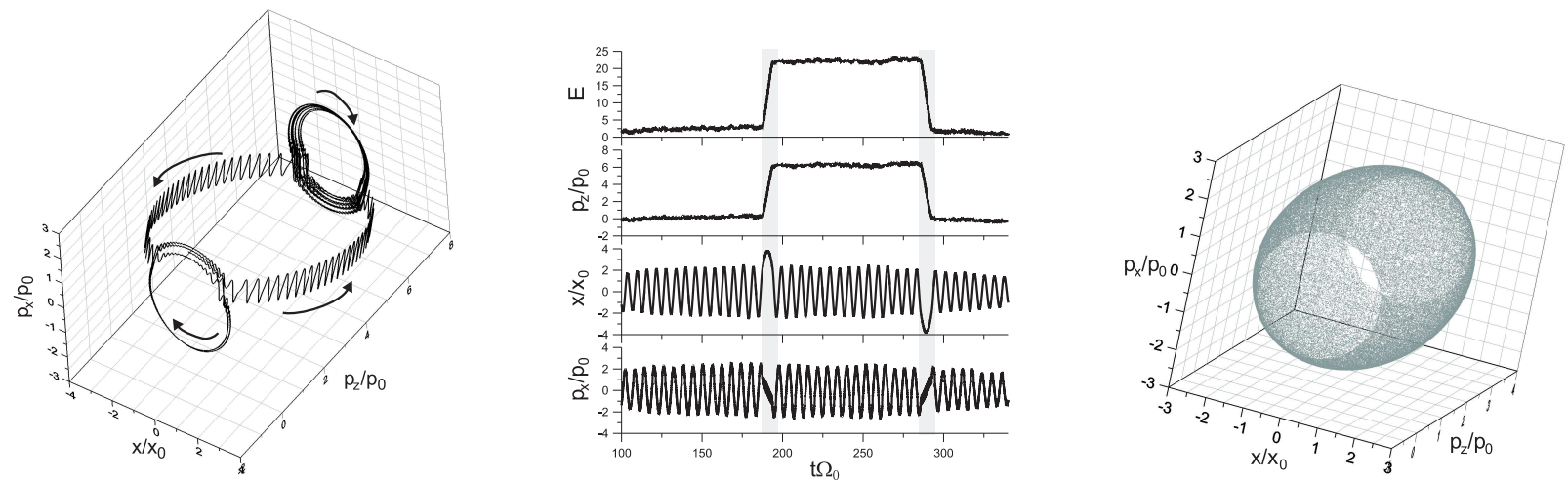

Figure 22: Numerical simulations of Hamiltonian (125). Characteristic trajectory (left panel); energy, coordinate, and momenta (central panel). A stroboscopic map in the $\left(x, p_{x}, p_{z}\right)$ space showing multiple scatterings on resonance for Hamiltonian (125) (right panel). Variables are normalized by $x_{0}=\sqrt{h_{0} / m} / \Omega_{0}, p_{0}=\sqrt{h_{0} m}$. The figure is adopted from [137].

All the energy gain is in the longitudinal component of the momentum. After several of Larmor periods with $p_{z}$ close to $p_{z}^{(1)}$, the particle can be trapped into resonance again. This time the trapping occurs at $x<0$, because $d|x| / d t>0$ at $x<0$ on this part of $\mathcal{R}$. After the trapping, the particle moves along the resonant curve and escapes from resonance near $\mathcal{L}_{0}$, thus returning close to its initial energy (Fig. 22(right panel)).

In the coordinate space, trapped particles move along the wave front. For the first trapping, this motion can be divided into two stages. During the first stage, $x$ grows with time and particles experience the surfatron acceleration similar to Subsect. 6.2. If $k_{z} / k \ll 1$ (i.e., $\mathbf{k}$ is almost perpendicular to the background magnetic field), the maximum value of $|x|$ on the resonant curve is $|x|_{\max } \approx v_{\phi} k / \Omega_{0} k_{z}$, and trapped particles are significantly accelerated. It was shown in Subsect. 6.2, that if $k_{z}=0$ the surfatron acceleration is unlimited (the duration of the first stage in infinite). However, if $k_{z} \neq 0$, the first stage is finite and during the second stage $x$ decreases to its initial value. The particle gains energy in the $z$-direction (motion along the background magnetic field) during both stages: $p_{z}$ grows in resonance (see Fig. 22(central panel, left grey zone)). After escaping from resonance, a particle makes several gyrorotations and becomes trapped again. This second trapping motion corresponds to $p_{z}$ decreasing in resonance (see Fig. 22(central panel, right grey zone)).

Scattering on resonance produces diffusion of the particle energy even when trapping is impossible. A stroboscopic map with a period $2 \pi / \omega$ of a long phase trajectory with multiple scatterings on resonance is shown in Fig. 22(central panel). The dots uniformly cover a large part of the isoenergetic ellipsoid $H=h$. The two regions not covered by dots around the poles correspond to Larmor trajectories that never cross the resonant plane. Motion there is regular.

To investigate the evolution of particle velocity distributions we performed a simulation of $10^{6}$ test particles. The initial distribution was uniform in the $\left(x, p_{x}\right)$ plane and all particles had approximately the same values of $p_{z} \sim 0$. In the $\left(p_{x}, p_{z}\right)$ plane, such a distribution looks like a narrow stripe $p_{z} \approx$ const. After a certain time $\left(t \Omega_{0} \sim 60\right)$, the distribution becomes wider in $p_{z}$ due to the scattering. Moreover, some particles are trapped; for these particles the $p_{z}$-component of momentum grows with time. Therefore, in the $\left(p_{x}, p_{z}\right)$ plane there is a smooth cloud of particles with small $p_{z}$ and a stripe of trapped particles (Fig. 23, $t=t_{1}$ ). Then some of the trapped particles escape from resonance and fill the region with large $p_{z}$ (Fig. 23, $t=t_{2}$ ). This region spreads due to the scattering (Fig. 23, $t=t_{3}$ ). After a long enough time we observe three groups of particles in the $\left(p_{x}, p_{z}\right)$ plane: two groups with a relatively similar number of particles (with small and large $p_{z}$ ) and a stripe filled with captured particles.

Section summary. In this section we considered four nonrelativistic systems containing resonant interaction. Although systems describe significantly different configurations of electromagnetic fields, we show that the same approach can be applied for their description. For each system we derived the resonant Hamiltonian $H_{\phi}$ and computed the average change of particle energy due to trapping. These four examples cover a wide range of space plasma systems where the resonant wave-particle interaction plays an important role for charged particle dynamics. 

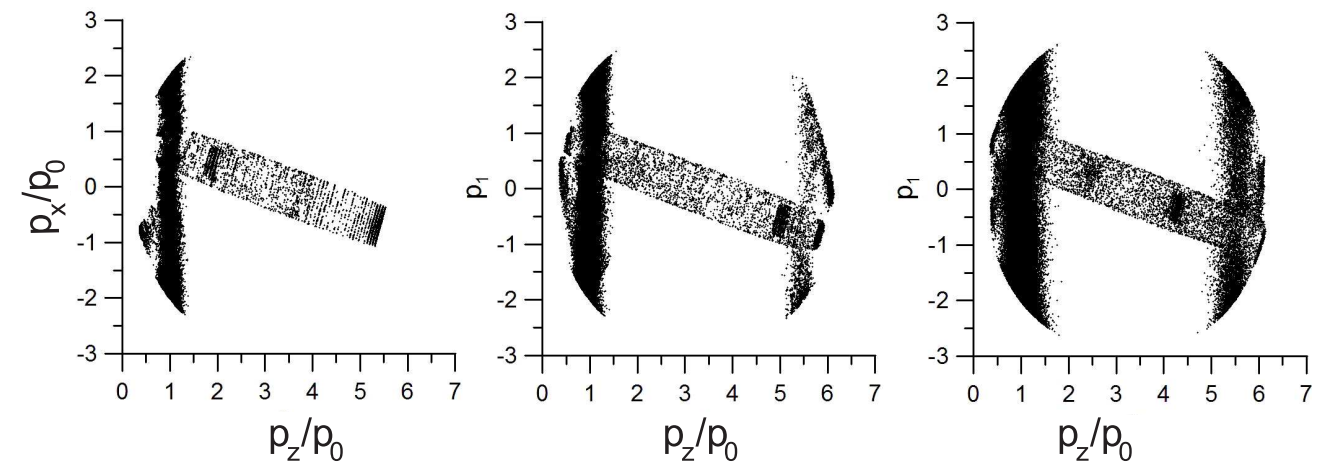

Figure 23: The time evolution of particle distribution in momentum space $\left(p_{x}, p_{z}\right)$. The panels correspond to $t_{i} \Omega_{0}=60,120,360$. Variables are normalized on $x_{0}=\sqrt{h_{0} / m} / \Omega_{0}, p_{0}=\sqrt{h_{0} m}$. Figure is adopted from [137].

\section{Particle acceleration in relativistic plasma systems}

In this section we discuss three settings with trapping of relativistic particles. In Subsect. 7.1 we describe trapping in the same system as in Subsect. 6.1 but for relativistic particles and show how relativistic effects change the dynamics of trapped particles. Two other subsections discusses charged particle (electron) resonant interaction with whistler waves in inhomogeneous magnetic field, which is a classical problem for near-Earth space plasma physics. We consider two limits: very oblique propagation of a whistler wave relative to the background magnetic field (in this case, the wave is almost electrostatic and can trap electrons into the Landau resonance), and a parallel propagation of a whistler wave, when the wave is purely electromagnetic and can trap electrons into the cyclotron resonance.

As in Sect. 6, we restrict our consideration to 2D physical systems. Keeping the same configuration of the background magnetic field, as shown in Fig. 16, we obtain a relativistic Hamiltonian (cf. Eq. (98))

$$
H=\sqrt{m^{2} c^{4}+c^{2} p_{x}^{2}+c^{2} p_{z}^{2}+e^{2} A_{y}^{2}(x, z)}=m c^{2} \gamma
$$

The corresponding Hamiltonian equations are

$$
\begin{aligned}
\dot{x} & =\frac{\partial H}{\partial p_{x}}=\frac{1}{m \gamma} p_{x}, \quad \dot{z}=\frac{\partial H}{\partial p_{z}}=\frac{1}{m \gamma} p_{z} \\
\dot{p}_{x} & =-\frac{\partial H}{\partial x}=\frac{e^{2}}{m c^{2} \gamma} B_{0} A_{y} \\
\dot{p}_{z} & =-\frac{\partial H}{\partial z}=-\frac{e^{2}}{m c^{2} \gamma} B_{0 x} A_{y}
\end{aligned}
$$

Comparing Eqs. (137) and Eqs. (99), we obtain for $B_{0}=$ const (in which case $B_{0 x}=0$ ) the new gyrofrequency $\Omega_{0} / \gamma$ where $\Omega_{0}=e B_{0} / m c$ is a gyrofrequency in the nonrelativistic $(\gamma \rightarrow 1)$ case.

\subsection{Electrostatic wave propagating across a weak magnetic field}

This subsection generalizes results derived in Subsect. 6.1 to relativistic particles. The upper boundary of particle velocity limits the background Lorentz force. If the wave electrostatic field exceeds the maximum Lorentz force, the particle trapped motion (and acceleration) becomes infinitely long [44, 138, 139]. Analysis of similar systems with electromagnetic waves can be found in $[119,140,110]$, and effects of curved magnetic field lines were considered in [141]. Effects of the 2-D fronts of waves (curvature of the wave front) were considered [142, 143].

A relativistic electron moving in a background uniform magnetic field $B_{0}$ and a wave electrostatic field is described by a Hamiltonian

$$
\begin{array}{r}
H=m c^{2} \gamma+e \Phi_{0} \sin \phi \\
\gamma=\sqrt{1+\left(\frac{p_{x}}{m c}\right)^{2}+\left(\frac{e B_{0} x}{m c^{2}}\right)^{2}}
\end{array}
$$


where $\Phi_{0}$ is an amplitude of the scalar potential, $\phi=\phi_{0}+k x-\omega t$ is a wave phase, $\omega \gg \Omega_{0}=e B_{0} / m c$, and $v_{\phi}=\omega / k=$ const $<c$ is a phase velocity. The corresponding Hamiltonian equations are (cf. Eq. (101)):

$$
\dot{x}=p_{x} / m \gamma, \quad \dot{p}_{x}=-\frac{m \Omega_{0}^{2} x}{\gamma}-e k \Phi_{0} \cos \phi
$$

Following Eqs. (7)-(9), we introduce momentum $I$ conjugate to $\phi$ by generating function (cf. Eq. (7))

$$
R=I(k x-\omega t)+P_{x} x
$$

where $\left(P_{x}, x\right),(I, \phi)$ are new variables

$$
p_{x}=\frac{\partial R}{\partial x}=P_{x}+I k
$$

The new Hamiltonian takes the form (cf. Eq. (9))

$$
H=-\omega I+\sqrt{1+\left(\frac{P_{x}+k I}{m c}\right)^{2}+\left(\frac{e B_{0} x}{m c^{2}}\right)^{2}}+e \Phi_{0} \sin \phi
$$

The resonant condition $\dot{\phi}=0$ can be written as

$$
\begin{aligned}
\frac{\partial H}{\partial I} & =-\omega+\frac{k}{\gamma}\left(\frac{P_{x}+k I}{m}\right)=0 \\
\gamma & =\sqrt{1+\left(\frac{P_{x}+k I}{m c}\right)^{2}+\left(\frac{\Omega_{0} x}{c}\right)^{2}}
\end{aligned}
$$

Solution of Eq. (143) is (cf. Eq. (17)):

$$
I_{\text {res }}=\frac{1}{k}\left(m c \frac{v_{\text {res }} \sqrt{1+\left(\Omega_{0} x / c\right)^{2}}}{\sqrt{1-v_{\text {res }}^{2}}}-P_{x}\right)
$$

where $v_{\text {res }}=\dot{\phi} / c=\omega / k c=$ const. Using Eqs. $(33,34)$ we can write the Hamiltonian near the resonance:

$$
\begin{aligned}
H_{\phi} & =\frac{1}{2} g(x) P_{\phi}^{2}-r(x) \phi+e \Phi_{0} \sin \phi \\
g & =\left.\frac{\partial^{2} H}{\partial I^{2}}\right|_{I=I_{\text {res }}}=\frac{\left(1-v_{r e s}^{2}\right)^{3 / 2} m k^{2}}{\sqrt{1+\left(\Omega_{0} x / c\right)^{2}}} \\
r & =-\frac{\Omega_{0}^{2} m x}{k} \frac{1}{\sqrt{1-v_{r e s}^{2}} \sqrt{1+\left(\Omega_{0} x / c\right)^{2}}}
\end{aligned}
$$

where $P_{\phi}=\dot{\phi} / g$ is a momentum conjugate to the phase $\phi$. The area $S_{\text {res }}$ is defined by Eqs. (40) and (43):

$$
S_{\text {res }}=\frac{2}{1-v_{\text {res }}^{2}} \frac{\Omega_{0}}{k^{2}} \sqrt{2 k|x|} f_{s}(a), \quad a=-\frac{e k \Phi_{0}}{m \Omega_{0}^{2} x} \sqrt{1-v_{\text {res }}^{2}} \sqrt{1+\left(\Omega_{0} x / c\right)^{2}}
$$

For high-energy particles (e.g., for $\Omega_{0} x / c \gg 1$ ) we have

$$
a=-\frac{e k \Phi_{0}}{m c \Omega_{0}} \sqrt{1-v_{r e s}^{2}}=\text { const }
$$

If $|a|>1, S_{\text {res }}$ grows with $x$. This is a crucial difference between the relativistic particle interaction with electrostatic wave and a corresponding nonrelativistic system, where $S_{\text {res }}$ given by Eq. (104) drops to zero for large $x$. Figure 24 presents an example of a trajectory of a particle that is trapped by the electrostatic wave and does not escape from 

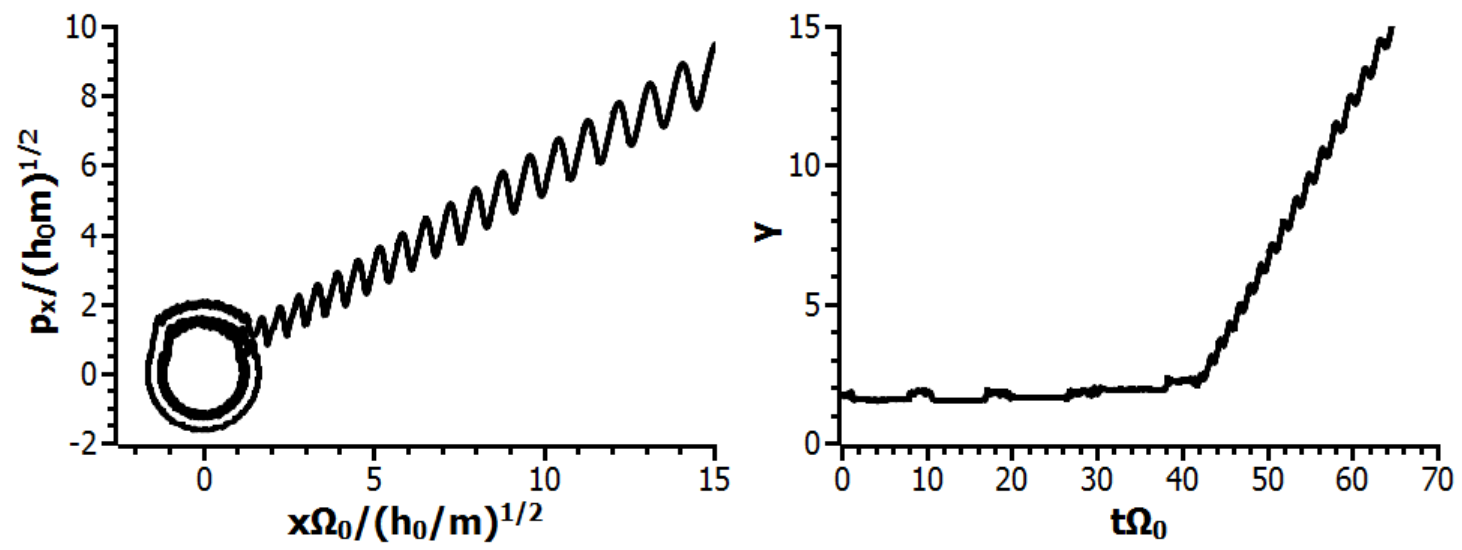

Figure 24: Trajectory and the corresponding energy from a numerical simulation of (139).

resonance (cf. Fig.17). The growth of $S_{\text {res }}$ for positive $x$ (see Sect. 3) results in particle trapping at the right-hand side of the closed trajectory in the $\left(x, p_{x}\right)$ plane. The trapped particle starts moving along the resonant trajectory

$$
p_{x}=\frac{v_{r e s}}{\sqrt{1-v_{r e s}^{2}}} \sqrt{1+\left(\Omega_{0} x / c\right)^{2}}
$$

with increasing $x$ and $p_{x}$. The trapped particle energy increases linearly with time $\gamma=\sqrt{1+\left(p_{x} / m c\right)^{2}+\left(\Omega_{0} x / c\right)^{2}} \sim$ $\sqrt{1+\left(\Omega_{0} t\right)^{2}} \sim t$. This type of acceleration is called surfatron acceleration [44, 138, 106, 144, 145].

Using Eq. (64) we estimate a change of particle momentum $p_{x}$ due to scattering on resonance in the relativistic system (cf. Eq. (105)):

$$
\left\langle\Delta p_{x}\right\rangle=-\left(1-v_{r e s}^{2}\right)^{3 / 2} \sqrt{\frac{2}{k|x|}} \frac{e k \Phi_{0}}{\Omega_{0}} f_{p}
$$

The corresponding energy change $\Delta h$ is

$$
\Delta h=\frac{p_{x}}{m}\left|\left\langle\Delta p_{x}\right\rangle\right|=e \Phi_{0} \gamma_{0}\left(1-v_{\text {res }}^{2}\right)^{3 / 2} \frac{\omega}{\Omega_{0}} \sqrt{\frac{2}{k\left|x_{\text {trap }}\right|}} f_{p}
$$

where $\gamma_{0}$ is initial particle energy, and $x_{\text {trap }}$ is a coordinate of resonance, which can be calculated from Hamiltonian (138) with resonant condition $p_{x}=\gamma v_{\text {res }} m c$ :

$$
\Omega_{0}\left|x_{\text {trap }}\right|=c \sqrt{\gamma_{0}^{2}\left(1-v_{r e s}^{2}\right)-1}
$$

For a sufficiently large wave amplitude $(a \gg 1), f_{p} \sim 8 \sqrt{2 / a}$ and Eq. (150) takes a form

$$
\Delta h=4 \gamma_{0} \sqrt{e \Phi_{0} v_{\phi}^{2} m} \frac{\left(1-v_{\text {res }}^{2}\right)^{5 / 4}}{\left(1+\left(\Omega_{0} x_{\text {trap }} / c\right)^{2}\right)^{1 / 4}}=4 \sqrt{e \Phi_{0} \gamma_{0} v_{\phi}^{2} m}\left(1-\frac{v_{\phi}^{2}}{c^{2}}\right)
$$

where $v_{\phi}=\omega / k=v_{r e s} c$. Comparison of Eq. (152) with the analogous expression for nonrelativistic system Eq. (105) shows that there is an additional factor $\sqrt{\gamma_{0}}\left(1-\left(v_{\phi} / c\right)^{2}\right)$. Therefore the amplitude of energy change and the resonance width in energy space are larger for $\gamma_{0}>1$ and rapidly decrease when $v_{\phi}$ reaches speed of light. 


\subsection{The Landau resonance in an inhomogeneous magnetic field}

This subsection generalises results derived in Subsect. 6.3 for relativistic systems, but instead of localised wave pulses we consider a periodic wave. We consider electron trapping into the Landau resonance with an electrostatic mode of a whistler wave propagating in an inhomogeneous magnetic field [146, 147]. Generalization of this system for more sophisticated resonant conditions can be found in [148], effects of curved magnetic field lines were considered in $[149,150]$. The Hamiltonian of a relativistic electron is (see Eq. (136) and [146, 151])

$$
\begin{aligned}
H & =m c^{2} \gamma+e \Phi_{0} u(z) \sin \phi \\
\gamma & =\sqrt{1+\frac{p_{x}^{2}+p_{z}^{2}}{m^{2} c^{2}}+\left(\frac{e B_{0}(z)}{m c^{2}} x\right)^{2}}
\end{aligned}
$$

where function $u(z)$ defines the spatial distribution of the wave field. The general form of wave-phase in this case is

$$
\phi=\int k(\tilde{z}) d \tilde{z}-\omega t+n \Omega_{0}
$$

where $n$ is a resonance number. We focus on the Landau resonance, $n=0$, and consider a strong background magnetic field $\Omega_{0}(z)=e B_{0}(z) / m c>\omega$. Hamiltonian equations of unperturbed system, $\Phi_{0} \sim 0$, describe a nonlinear oscillator:

$$
\dot{x}=p_{x} / m \gamma, \quad \dot{p}_{x}=-\Omega_{0}^{2} x m / \gamma
$$

We introduce new variable $I_{x}$ (see details in Appendix B):

$$
I_{x}=\frac{1}{2 \pi} \oint p_{x} d x=\frac{m c}{2 \pi} \oint \sqrt{\gamma^{2}-\frac{p_{z}^{2}}{m^{2} c^{2}}-\left(\frac{\Omega_{0}(z)}{c} x\right)^{2}} d x=\frac{m c^{2}}{2 \Omega_{0}(z)}\left(\gamma^{2}-1-\frac{p_{z}^{2}}{m^{2} c^{2}}\right)
$$

The Hamiltonian can be written as

$$
\begin{aligned}
H & =m c^{2} \gamma+e \Phi_{0} u(z) \sin \phi \\
\gamma & =\sqrt{1+\frac{p_{z}^{2}}{m^{2} c^{2}}+\frac{2 \Omega_{0}(z) I_{x}}{m c^{2}}}
\end{aligned}
$$

Here $I_{x}$ is a particle magnetic moment and is conserved in the absence of perturbations that depend on $x$. We use a generating function $R$ to introduce the phase $\phi$ as a new variable (see details in Eqs. (7)-(9)):

$$
R=I\left(\int k(\tilde{z}) d \tilde{z}-\omega t\right)+P_{z} z
$$

Therefore, the old momentum $p_{z}$ is

$$
p_{z}=\frac{\partial R}{\partial z}=P_{z}+I k(z)
$$

In the new variables the Hamiltonian is

$$
\begin{aligned}
H & =-\omega I+m c^{2} \gamma+e \Phi_{0} u(z) \sin \phi \\
\gamma & =\sqrt{1+\frac{\left(P_{z}+k I\right)^{2}}{m^{2} c^{2}}+Y} \quad Y=\frac{2 \Omega_{0}(z) I_{x}}{m c^{2}}
\end{aligned}
$$

Resonant condition $\dot{\phi}=0$ for Hamiltonian (160) is given by equation

$$
\frac{\partial H}{\partial I}=-\omega+\frac{k}{m \gamma}\left(P_{z}+k I\right)=0
$$



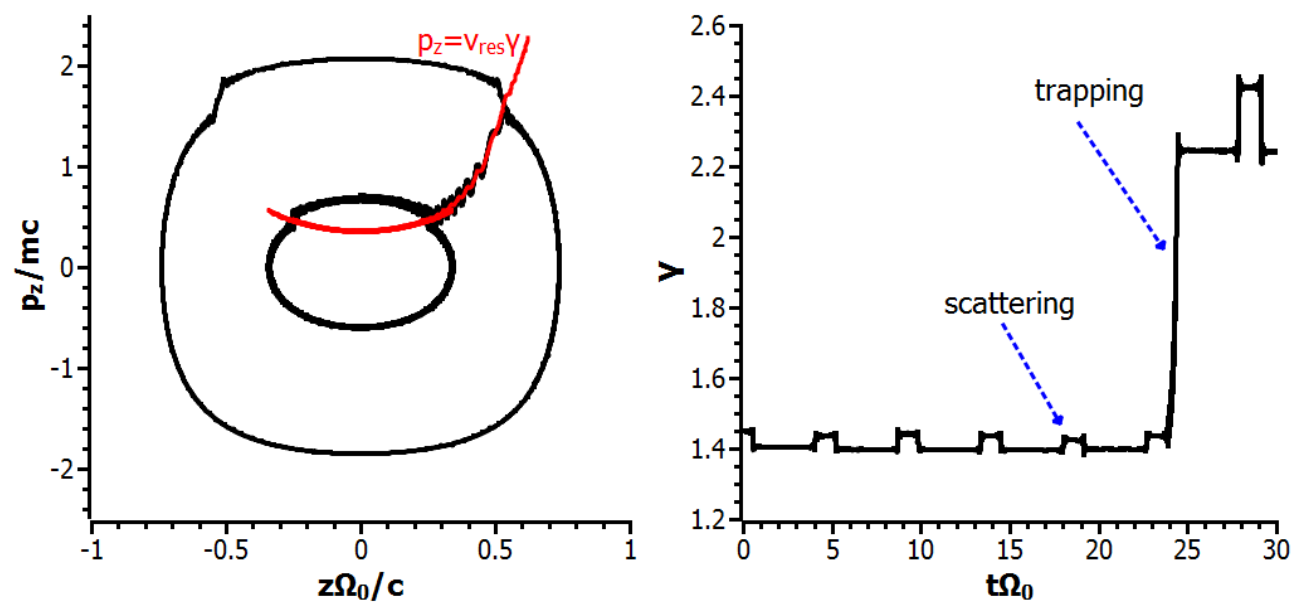

Figure 25: A trajectory and the corresponding energy variation obtained by a numerical integration of (157). Magnetic field is approximated by a dipole field $B_{0}(z)$ [112], and $u(z)=z^{2} /\left(z^{2}+0.02\right)$ function is defined from a whistler wave model [146]

Solution of Eq. (161) is (cf. Eq. (144)):

$$
I_{\text {res }}=\frac{1}{k}\left(m c \frac{v_{\text {res }} \sqrt{1+Y^{2}}}{\sqrt{1-v_{r e s}^{2}}}-P_{z}\right)
$$

where $v_{\text {res }}=v_{\phi} / c=\omega / k(z) c$ depends on $z$. Using Eqs. $(33,34)$ we can write the Hamiltonian near the resonance as

$$
\begin{aligned}
H_{\phi} & =\frac{1}{2} g(x) P_{\phi}^{2}-r(x) \phi+e \Phi_{0} u(z) \sin \phi \\
g & =\left.\frac{\partial^{2} H}{\partial I^{2}}\right|_{I=I_{\text {res }}}=\frac{\left(1-v_{\text {res }}^{2}\right) m k^{2}}{\gamma_{\text {res }}} \\
r & =m c^{2}\left\{\gamma_{\text {res }}, I_{\text {res }}\right\}=-\frac{m c^{2} W}{\gamma_{\text {res }}\left(1-v_{\text {res }}^{2}\right)} \\
W & =k^{-1}\left(Y Y^{\prime}+\gamma_{\text {res }}^{2} v_{\text {res }} v_{\text {res }}^{\prime}\right), \quad \gamma_{\text {res }}=\frac{\sqrt{1+Y}}{\sqrt{1-v_{r e s}^{2}}}
\end{aligned}
$$

where $P_{\phi}=\dot{\phi} / g$ is a momentum conjugate to the phase $\phi, Y^{\prime}=\left(d \Omega_{0} / d z\right) I_{x} / m c^{2}, v_{r e s}^{\prime}=d v_{r e s} / d z$. The area $S_{r e s}$ is defined by Eqs. (40):

$$
S_{r e s}=\frac{2 m c \sqrt{2 W}}{\left(1-v_{r e s}^{2}\right) k} f_{s}(a), \quad a=-\frac{e \Phi_{0} u}{m c^{2}} \frac{\gamma_{r e s}\left(1-v_{r e s}^{2}\right)}{W}
$$

Using Eq. (47) we can write expression for probability of trapping:

$$
\Pi \approx \frac{\gamma_{r e s} v_{r e s}\left(1-v_{r e s}^{2}\right)}{2 \pi W} \frac{1}{m c} \frac{\partial S_{r e s}}{\partial z}
$$

Example of a particle trajectory in system (157) is shown in Fig. 25. The particle moves around a closed trajectory in the $\left(z, p_{z}\right)$ plane. The area $S_{\text {res }}$ grows for positive $z$, where the particle becomes trapped and starts accelerating. At some distance from $z=0, S_{\text {res }}$ starts decreasing and the trapped particle escapes from resonance. After leaving the resonance, the particle starts moving along a closed trajectory with a larger radius. The increase of oscillation amplitude in the $\left(z, p_{z}\right)$ plane corresponds to the increase of particle energy due to the acceleration in trapping. 


\subsection{The cyclotron resonance in an inhomogeneous magnetic field}

In this subsection we consider the cyclotron resonance between relativistic electrons $(\gamma>2)$ and a whistler wave propagating along an inhomogeneous magnetic field. The Resonance condition for such systems, $p_{z} k / m=\omega \gamma-\Omega$ depends on the gyrofrequency $\Omega[152,153]$ and is significantly different from the Cherenkov $(\mathbf{p k} / m=\omega \gamma)$ or the Landau $\left(p_{z} k / m=\omega \gamma\right)$ resonances.

An electromagnetic whistler wave propagating parallel to the background magnetic field can be described by two components of a vector potential

$$
\begin{aligned}
A_{y} & =A \sin \phi, \quad A_{x}=-A \cos \phi \\
\phi & =\int k(\tilde{z}) d \tilde{z}-\omega t
\end{aligned}
$$

Hamiltonian (136) is

$$
H=\sqrt{m^{2} c^{4}+p_{z}^{2} c^{2}+\left(c p_{x}-e A \cos \phi\right)^{2}+\left(e B_{0} x+e A \sin \phi\right)^{2}}
$$

Assuming that wave energy $e A$ is much smaller than the particle rest energy $m c^{2}$, we can expand Hamiltonian (167):

$$
\begin{aligned}
H & =m c^{2} \gamma+\frac{e A}{\gamma m c}\left(\Omega_{0} m x \sin \phi-p_{x} \cos \phi\right) \\
\gamma & =\sqrt{1+\frac{p_{x}^{2}+p_{z}^{2}}{m^{2} c^{2}}+\left(\frac{\Omega_{0} x}{c}\right)^{2}}
\end{aligned}
$$

where $\Omega_{0}(z)=e B_{0}(z) / m c$. For unperturbed system $(A \sim 0)$ we can introduce a new variable $I_{x}$ (see Eq. (156) and Appendix B):

$$
I_{x}=\frac{1}{2 \pi} \oint p_{x} d x=\frac{m c^{2}}{2 \Omega_{0}}\left(\gamma^{2}-1-\frac{p_{z}^{2}}{m^{2} c^{2}}\right)
$$

Coordinates $\left(x, p_{x}\right)$ can be rewritten using $I_{x}$ and a conjugate variable $\psi$ :

$$
x=\sqrt{2 I_{x} / m \Omega_{0}} \sin \psi, \quad p_{x}=\sqrt{2 I_{x} m \Omega_{0}} \cos \psi
$$

Substituting Eqs. (170) into Eq. (168) we obtain

$$
\begin{aligned}
H & =m c^{2} \gamma-\frac{e A}{\gamma} \sqrt{Y} \cos (\phi+\psi) \\
\gamma & =\sqrt{1+\frac{p_{z}^{2}}{m^{2} c^{2}}+Y,} \quad Y=\frac{2 I_{x} \Omega_{0}}{m c^{2}}
\end{aligned}
$$

Corresponding Hamiltonian equations are

$$
\begin{aligned}
\dot{I}_{x} & =-\frac{\partial H}{\partial \psi}=-\frac{e A}{\gamma} \sqrt{Y} \sin (\phi+\psi) \\
\dot{\psi} & =\frac{\partial H}{\partial I_{x}}=\frac{\Omega_{0}}{\gamma}-\frac{e A}{2 I_{x} \gamma} \sqrt{Y} \cos (\phi+\psi) \\
\dot{p}_{z} & =-\frac{\partial H}{\partial z}=-\frac{1}{\gamma} \frac{\Omega_{0}^{\prime}}{2 \Omega_{0}} Y-\frac{e k A}{\gamma} \sqrt{Y} \sin (\phi+\psi)-\frac{e A}{\gamma} \frac{\Omega_{0}^{\prime}}{2 \Omega_{0}} \sqrt{Y} \cos (\phi+\psi) \\
\dot{z} & =\frac{\partial H}{\partial p_{z}}=\frac{p_{z}}{m \gamma}
\end{aligned}
$$

We use the following generating function to introduce new phase $\zeta=\phi+\psi$ and conjugate momentum $I$ :

$$
R=I\left(\psi+\int k(\tilde{z}) d \tilde{z}-\omega t\right)+P_{z} z+\tilde{I}_{x} \psi
$$


where $\left(z, P_{z}\right),\left(\psi, \tilde{I}_{x}\right)$ are new variables: $p_{z}=k I+P_{z}, I_{x}=I_{x}+I$. The new Hamiltonian takes the form:

$$
\begin{aligned}
H & =-\omega I+m c^{2} \gamma-\frac{e A}{\gamma} \sqrt{Y} \cos \zeta \\
\gamma & =\sqrt{1+\frac{\left(k I+P_{z}\right)^{2}}{m^{2} c^{2}}+Y}, \quad Y=\frac{2\left(\tilde{I}_{x}+I\right) \Omega_{0}}{m c^{2}}
\end{aligned}
$$

Hamiltonian (174) does not depend on $\psi$ anymore. Thus, the conjugate momentum $\tilde{I}_{x}$ is conserved and can be set to zero: $Y=2 I \Omega_{0} / m c^{2}$. The resonant condition for Hamiltonian (174) becomes

$$
\dot{\zeta}=\frac{\partial H}{\partial I}=-\omega+\frac{1}{\gamma}\left(\Omega_{0}+k \frac{k I+P_{z}}{m}\right)=0
$$

Solution of Eq. (175) is

$$
\frac{k I_{\text {res }}}{m c}=\frac{v_{\text {res }}}{\sqrt{1-v_{\text {res }}^{2}}} \sqrt{1-\left(\frac{\Omega_{0}}{k c}\right)^{2}-2 \frac{\Omega_{0}}{k c} \frac{P_{z}}{m c}}-\frac{\Omega_{0}}{k c}-\frac{P_{z}}{m c}
$$

where $v_{\text {res }}(z)=v_{\phi}(z) / c=\omega / k(z) c$. The corresponding resonant energy $\gamma_{\text {res }}$ is

$$
\gamma_{\text {res }}=\frac{1}{\sqrt{1-v_{\text {res }}^{2}}} \sqrt{1-\left(\frac{\Omega_{0}}{k c}\right)^{2}-2 \frac{\Omega_{0}}{k c} \frac{P_{z}}{m c}}
$$

We expand Hamiltonian (174) around the resonance $I=I_{\text {res }}$ :

$$
\begin{aligned}
H & =m c^{2} \Lambda+\frac{1}{2}\left(I-I_{\text {res }}\right)^{2}-\frac{e A}{\gamma_{\text {res }}} \sqrt{Y_{\text {res }}} \cos \zeta \\
\Lambda & =-\frac{\omega I_{\text {res }}}{m c^{2}}+\gamma_{\text {res }}=\frac{1}{v_{\text {res }}}\left(\frac{\Omega_{0}}{k c}+\frac{P_{z}}{m c}\right) \\
Y_{\text {res }} & =\frac{2 I_{\text {res }} \Omega_{0}}{m c^{2}}=2 \frac{\Omega_{0}}{k c}\left(v_{\text {res }} \gamma_{\text {res }}-\frac{\Omega_{0}}{k c}-\frac{P_{z}}{m c}\right) \\
g & =\left.\frac{\partial^{2} \gamma}{\partial I^{2}}\right|_{I=I_{\text {res }}}=\frac{k^{2}}{\gamma_{\text {res }} m}\left(1-v_{\text {res }}^{2}\right)
\end{aligned}
$$

We introduce $I-I_{\text {res }}$ as a new variable $P_{\phi}=\dot{\phi} / g$ and rewrite the resonance Hamiltonian as (see Eqs. $(33,34)$ ):

$$
\begin{aligned}
H_{\phi} & =\frac{1}{2} g P_{\phi}^{2}-r \phi-\frac{e A}{\gamma_{r e s}} \sqrt{Y_{\text {res }}} \cos \zeta \\
r & =m c^{2}\left\{\gamma_{\text {res }}, I_{\text {res }}\right\}=\frac{m c^{2} W}{\gamma_{\text {res }}\left(1-v_{\text {res }}^{2}\right)} \\
W & =\frac{1}{k}\left(v_{R}^{2} \frac{k^{\prime}}{k}+\frac{\Omega_{0}}{k c} \frac{\Omega_{0}^{\prime}}{\Omega_{0}} \frac{P_{z}}{m c}\right)
\end{aligned}
$$

where $v_{R}=v_{\text {res }} \gamma_{\text {res }}-\Omega_{0} / k c=\left(\omega \gamma_{\text {res }}-\Omega_{0}\right) / k c$. The area $S_{\text {res }}$ is (see Eq. (40)):

$$
S_{\text {res }}=2 \frac{m c}{1-v_{\text {res }}^{2}} \frac{1}{k} \sqrt{2|W|} f_{s}(a), \quad a=-\frac{e A \sqrt{Y_{\text {res }}}}{\gamma_{\text {res }} r}=-\frac{e A}{m c^{2}} \frac{1-v_{\text {res }}^{2}}{W} \sqrt{Y_{\text {res }}}
$$

In Hamiltonian (179) and Eqs. (180) momentum $P_{z}$ equals to the resonant value $P_{\text {res }}(z)$, which is defined from unperturbed Hamiltonian (174) with initial energy $h_{\text {init }}$ :

$$
P_{\text {res }}=\frac{m c}{v_{\text {res }}}\left(\frac{h_{\text {init }}}{m c^{2}}-\frac{\Omega_{0}}{\omega}+\sqrt{1-v_{\text {res }}^{2}} \sqrt{1+\left(\frac{\Omega_{0}}{\omega}\right)^{2}-2 \frac{h_{\text {init }}}{m c^{2}} \frac{\Omega_{0}}{\omega}}\right)
$$



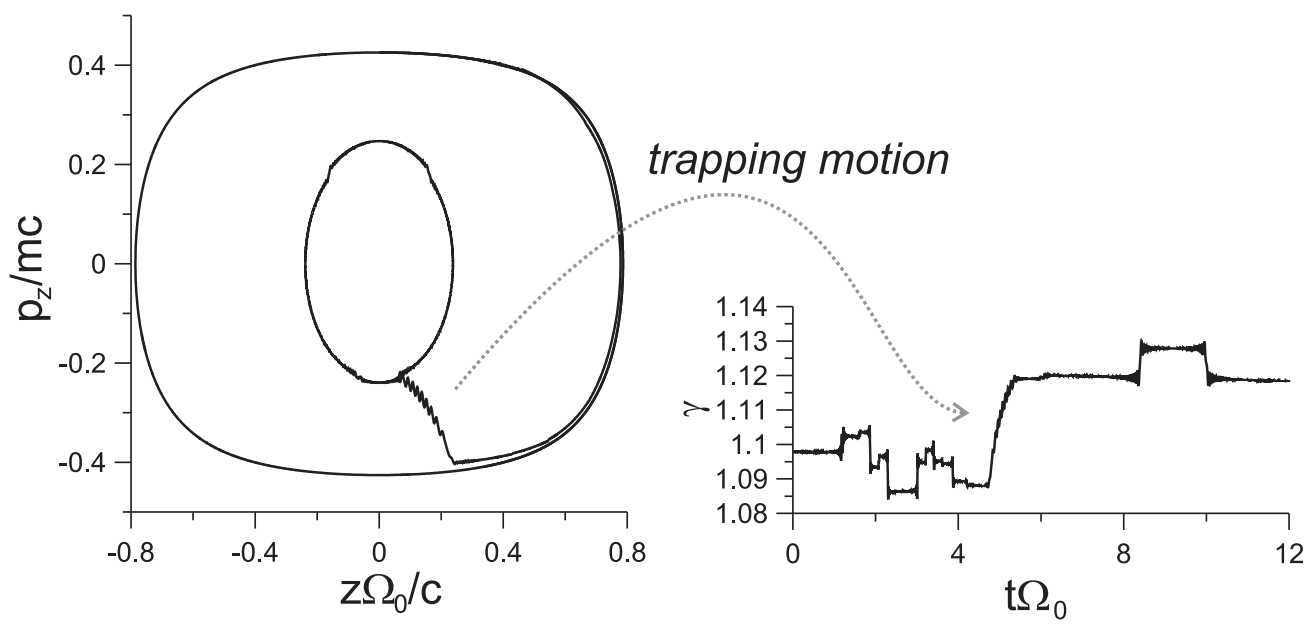

Figure 26: A trajectory and the corresponding energy variation obtained with a numerical integration of (172).

Substituting (181) to Eq. (177), we obtain $\gamma_{r e s}(z)$ along the trapped trajectory

$$
\gamma_{\text {res }}=\frac{1}{v_{\text {res }} \sqrt{1-v_{\text {res }}^{2}}}\left(\frac{\Omega_{0}}{\omega} \sqrt{1-v_{\text {res }}^{2}}-\sqrt{1+\left(\frac{\Omega_{0}}{\omega}\right)^{2}-2 \frac{\Omega_{0}}{\omega} \frac{h_{\text {init }}}{m c^{2}}}\right)
$$

A typical particle trajectory for Hamiltonian (171) is shown in Fig. 26. The particle oscillates in the $\left(z, p_{z}\right)$ plane and becomes trapped with a positive $z$. In contrast to the Landau resonance (see Fig. 25), trapping results in a decrease of the radius of particle trajectory in the $\left(z, p_{z}\right)$ plane. Near $z \sim 0$ a particle escapes from resonance with a net gain of energy and starts moving along a closed trajectory with a smaller radius.

Using Eq. (47) we have for the probability of trapping:

$$
\Pi \approx \frac{\left\{S_{r e s}, \Lambda\right\}}{2 \pi|r|}=\frac{1}{2 \pi m c} \frac{\gamma_{r e s}\left(1-v_{r e s}^{2}\right)}{v_{r e s}|W|}\left(\frac{\partial S_{r e s}}{\partial z}-\frac{\Omega_{0}^{\prime}}{\Omega_{0}} \frac{\Omega_{0}}{k c} m c \frac{\partial S_{r e s}}{\partial P_{z}}\right)
$$

where $\Lambda$ is defined by Eq. (178). Equation (183) shows that probability of trapping in 2D case (when $S_{r e s}=S_{r e s}\left(z, P_{z}\right)$ ) is much more complicated than for 1D system (e.g., the Landau resonance considered in Subsect. 7.2). A detailed investigation and numerical simulations of Eq. (183) were reported in [154].

Section summary. In this section we considered three relativistic systems with wave-particle resonant interaction. The relativistic effect changes the wave-particle resonance interaction by limiting the Lorentz force acting on particles. This problem is essentially important for description of electron acceleration in the near-Earth plasma environment.

\section{Stability of trapped particle motion}

This section discusses the stability of trapped particle motion in several systems described in Sects. 6, 7. Although in this section we consider only effects of an external nonresonant noise resulting in instability of trapped particle motion, the nonlinear resonant interaction can be destroyed by other types of instability. The most important one is the sideband instability leading to spread of wave spectra and stochastization of trapped particle motion due to resonances with satellite waves [e.g., 155, 17, 18]. Similar effects of particle motion stochastization and particle trapping destruction can provide several waves simultaneously resonating with particles [e.g., 156, 131, 157]. These effects are not considered in our review but should be analyzed and estimated for any plasma system where nonlinear wave-particle interaction is considered. We start with a general consideration of effects of fluctuations of background magnetic field on trapped particle motion, and then consider one particular system where such fluctuations result in the destruction of the wave-particle resonant interaction. 


\subsection{Destruction of the trapped motion invariant}

In this subsection we return to the systems considered in Subsect. 6.2, 7.1, 7.2 and show how magnetic field fluctuations can destroy the trapped particle motion and cause the diffusive escape of particles from resonance [158, 159]. We start with a consideration of general effects of magnetic field fluctuations on behavior of system (34), for which the particle dynamics in the $\left(\phi, P_{\phi}\right)$ plane has a Hamiltonian

$$
H_{\phi}=\frac{1}{2} g(x) P_{\phi}^{2}-r(x) \phi+\varepsilon u(x) \sin \phi
$$

In (184), $r(x), u(x)$, and $g(x)$ are functions of the coordinate along a resonance trajectory. Dynamics in the $\left(\phi, P_{\phi}\right)$ plane is described by Hamiltonian equations

$$
\dot{P}_{\phi}=r-\varepsilon u \cos \phi, \quad \dot{\phi}=g P_{\phi}
$$

Consider a particle trajectory oscillating near the bottom of the potential well in the $\left(\phi, P_{\phi}\right)$ plane. At the bottom, the potential energy $U=\Omega_{t r}^{2}\left(\sin \phi-(r / u \varepsilon) \phi\right.$ ) (where $\Omega_{t r}=\sqrt{\varepsilon u}$ ) has a minimum, yielding $\phi=\phi_{0}=\arccos (r / \varepsilon u)$. Expanding Hamiltonian (184) near $\phi_{0}$ we obtain

$$
H_{\phi}=\frac{1}{2} g P_{\phi}^{2}+\frac{1}{2} \Omega_{t r}^{2}\left(\phi-\phi_{0}\right)^{2}+\text { const }
$$

Equation (186) shows that $g \Omega_{t r}$ is frequency of trapped particle oscillations only at the bottom of the potential well in the $\left(\phi-\phi_{0}, P\right)$ plane. However, this term can be used to estimate the frequency of oscillations over almost the entire region filled by closed trajectories (except near the separatrix), because the actual frequency depends very weakly on the position of a particle (see, e.g., $[160,161])$. The period of trapped particle oscillations, $2 \pi /\left(g \Omega_{t r}\right)$, is small as compared with typical timescales of $x$ variation.

The periodicity of trapped particle motion in the $\left(\phi, P_{\phi}\right)$ plane allows us to introduce an action variable $I_{\phi}=$ $(2 \pi)^{-1} \oint P_{\phi} d \phi$ (see [162]). In a system with constant $x$, the particle trajectory in the $\left(\phi, P_{\phi}\right)$ plane does not evolve and, thus, the area surrounded by this particle trajectory is conserved exactly. In more realistic cases with slow variations of $x$, the action $I_{\phi}$ becomes an adiabatic invariant of $(184),[162,104]$, i.e., the area $2 \pi I_{\phi}$ is conserved to a high degree of accuracy even if the effective potential $U$ varies with $x$ (with time). Thus, $I_{\phi}$ can be used to characterize a trajectory of trapped particle motion. Consider a solution of Hamiltonian equations (185) for a particle initially trapped at $x=x_{0}$. As $x>x_{0}$ slowly changes, the particle motion in the $\left(\phi, P_{\phi}\right)$ plane is shown in Fig. 27. The action $I_{\phi}$ is calculated as

$$
I_{\phi}=\frac{1}{2 \pi} \oint P_{\phi} d \phi=\frac{1}{\pi} \sqrt{\frac{2}{g}} \int_{\phi_{1}}^{\phi_{2}} \sqrt{H_{\phi}+r \phi-\varepsilon u \sin \phi} d \phi
$$

where $\phi_{1,2}$ are to roots of the equation $H_{\phi}+r \phi-\varepsilon u \sin \phi=0$. The variations of parameters $g, r$, and $\varepsilon$ result in the observed evolution of the particle trajectory in the $\left(\phi, P_{\phi}\right)$ plane, but the action $I_{\phi}$ is conserved. There are only very small amplitude oscillations of $I_{\phi}$, corresponding to the fact that integral (187) is calculated for a frozen $x$, while there are actually small variations of $x$ within one period of trapped particle oscillations in the $\left(\phi, P_{\phi}\right)$ plane.

Let us consider a whole cycle of charged particle motion, including particle trapping and escape from resonance. We start the numerical integration at $x=0$, and select a trajectory which becomes trapped (see Fig. 28). We can use the conservation of $I_{\phi}$ for trapped particles to determine when a particle enters and escapes from resonance. At the moment of trapping, $2 \pi I_{\phi}=S_{\text {res }}$ (see Eq. (40)). Thus, the trapped particles escape from resonance when $S_{\text {res }}$ again becomes equal to $2 \pi I_{\phi}$. An example of particle escape from resonance is shown in Fig. 28.

Figure 28 demonstrate that in the trapped motion the adiabatic invariant $I_{\phi}$ is conserved in the original waveparticle system. However, if an additional (external) force inducing variations of $I_{\phi}$ is present, then $2 \pi I_{\phi}$ could become equal to $S_{\text {res }}$, leading to particle escape from resonance. In the next section, we consider the effects of such an external force and demonstrate that it may eventually lead to the destruction of the adiabatic invariant $I_{\phi}$.

The magnetic field fluctuations are mostly non-resonant: their frequencies, although of the same order as the main wave frequency, are assumed to differ sufficiently from it. Therefore we can neglect resonant interactions between particles trapped by the main wave and the fluctuations (in contrast to cases considered in $[18,157]$ ). Here we 

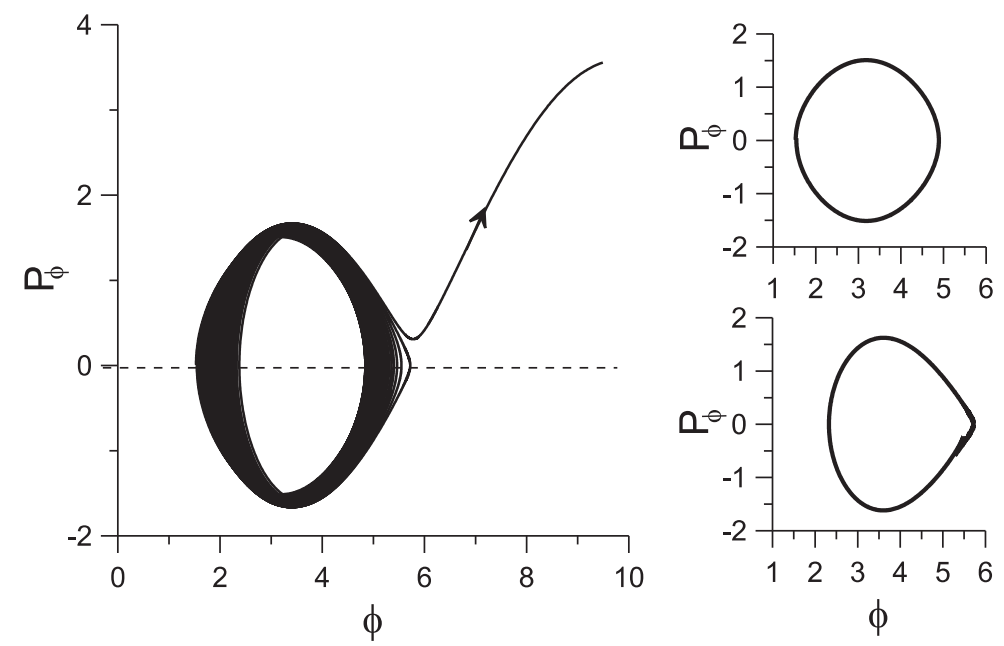

Figure 27: A characteristic particle trajectory in the $\left(\phi, P_{\phi}\right)$ plane. The right panels show two fragments of the trajectory (at the beginning and just before escape from resonance). The figure is adopted from [159].
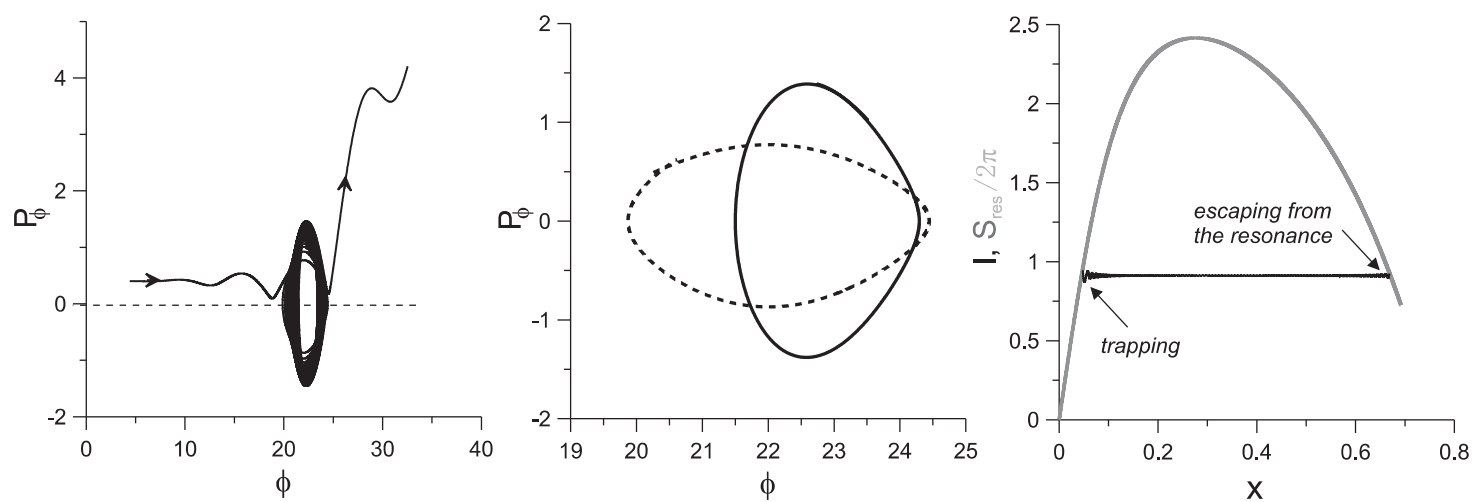

Figure 28: A trapped particle trajectory. The middle panel shows two fragments of the trajectory in the $\left(\phi, P_{\phi}\right)$ plane: just after trapping (dashed curve) and just before the escape (solid curve). $I_{\phi}$ and $S_{\text {res }}$ profiles are shown in the right panel. The Figure is adopted from [159]. 
consider only fluctuations parallel to the background field line (transverse fluctuations were briefly addressed in [16]). Such fluctuations can be included into the term $\sim r$ in Hamiltonian (184) as $\approx r_{1} \delta b$ with $\delta b$ being an amplitude of magnetic field fluctuations. In other words, $r$ can be expressed as a sum of unperturbed $r$ and perturbations $r_{1} \delta b$. The dependence of fluctuations on $x$ is included into the $r_{1}(x)$. Hamiltonian (184) takes the form

$$
H_{\phi}=\frac{1}{2} g P_{\phi}^{2}-r \phi+\varepsilon u \sin \phi-r_{1} \phi \delta b
$$

To keep our approach as general as possible, we do not specify the type of fluctuations $\delta b$ but simply introduce two parameters characterizing it: the timescale $\tau$ and variance $\operatorname{Var}(\delta b)=\sigma$. Magnetic field fluctuations are assumed to be high-frequency, such that $\tau \Omega_{t r} g \ll 1$, and both fast variables $\left(\phi, P_{\phi}\right)$ and slow variable $x$ change only weakly over one time step (time interval $\sim \tau$ ) of fluctuations.

As $\Omega_{t r} g=\partial H_{\phi} / \partial I_{\phi}$ (see [162]), we have for the change $\Delta I_{\phi}$ on one time step $(\sim \tau)$ of fluctuations:

$$
\Delta I_{\phi}=\frac{\Delta H_{\phi}}{\Omega_{t r} g}=-\frac{r_{1} \delta b}{\Omega_{t r} g} \Delta \phi
$$

where all functions on the right-hand side of Eq. (189) are evaluated at the same moment within one time step of fluctuations, while $\Delta \phi$ is the change of $\phi$ between the beginning and end of this step. This change $\Delta \phi$ over a small time step $\tau$ is $\Delta \phi=g P_{\phi} \tau$. Thus, Eq. (189) can be rewritten as

$$
\Delta I_{\phi}=-\frac{r_{1} \delta b}{\Omega_{t r}} P_{\phi} \tau
$$

On the right-hand side of Eq. (190), $\delta b$ changes much faster than $P_{\phi}$, and both $\delta b$ and $P_{\phi}$ change faster than $g(x)$, $\Omega_{t r}(x)$, or $r_{1}(x)$. One period of particle oscillations in the $\left(\phi, P_{\phi}\right)$ plane, $2 \pi / g \Omega_{t r}$, includes many steps $\sim \tau$, but is short enough to keep $x$ unchanged. Over this period the variance of $\Delta I_{\phi}$ is

$$
\operatorname{Var}\left(\Delta I_{\phi}\right)=\left(\frac{r_{1} \tau}{\Omega_{t r}}\right)^{2} \operatorname{Var}\left(P_{\phi} \delta b\right)
$$

We assume that fluctuations $\delta b$ and variations of $P_{\phi}$ are statistically independent and, thus, $\operatorname{Var}\left(\delta b P_{\phi}\right)=\sigma \operatorname{Var}\left(P_{\phi}\right)$. The term $\operatorname{Var}\left(P_{\phi}\right)$ can be considered as a sum of $M \gg 1$ values of $P_{\phi}^{2}$ calculated within consecutive time steps $\tau$ (i.e., between successive changes of $\delta b)$ :

$$
\operatorname{Var}\left(P_{\phi}\right)=\frac{1}{M} \sum_{i=0}^{M} P_{\phi, i}^{2}=\frac{\sum_{i=0}^{M} P_{\phi, i}^{2} \tau}{\sum_{i=0}^{M} \tau} \approx \frac{g \Omega_{t r}}{2 \pi} \oint P_{\phi}^{2} d t=\frac{\Omega_{t r}}{2 \pi} \oint P_{\phi} d \phi=\Omega_{t r} I_{\phi}
$$

where we took into account the smallness of $\tau$. Substituting Eq. (192) into Eq. (191), we obtain:

$$
\operatorname{Var}\left(\Delta I_{\phi}\right)=\frac{\left(r_{1} \tau\right)^{2}}{\Omega_{t r}} I_{\phi} \sigma
$$

The evolution of $I_{\phi}$ can be viewed as a random walk. We describe the behavior of an ensemble of such randomly walking trajectories (an ensemble of particles) in terms of the particle distribution function $\Psi\left(I_{\phi}, t\right)$ : the probability for a particle to have the value of adiabatic invariant in the interval $\left(I_{\phi}-\delta I_{\phi} / 2, I_{\phi}+\delta I_{\phi} / 2\right)$ at the time $t$ is given by $\Psi\left(I_{\phi}, t\right) \delta I_{\phi}$. For this distribution $\Psi\left(I_{\phi}, t\right)$ we can write a diffusion equation:

$$
\frac{\partial \Psi}{\partial t}=\frac{\partial}{\partial I_{\phi}}\left(D_{I I} \frac{\partial \Psi}{\partial I_{\phi}}\right)
$$

In Eq. (185), we introduced the diffusion coefficient $D_{I I}=\operatorname{Var}\left(\Delta I_{\phi}\right) / \tau$. Switching from $t$ to $x$ (via the Hamiltonian equation for system (30)) in Eq. (194) we get:

$$
\frac{\partial \Psi}{\partial h}=\frac{\partial}{\partial I_{\phi}}\left(I_{\phi} \frac{\partial \Psi}{\partial I_{\phi}}\right)
$$




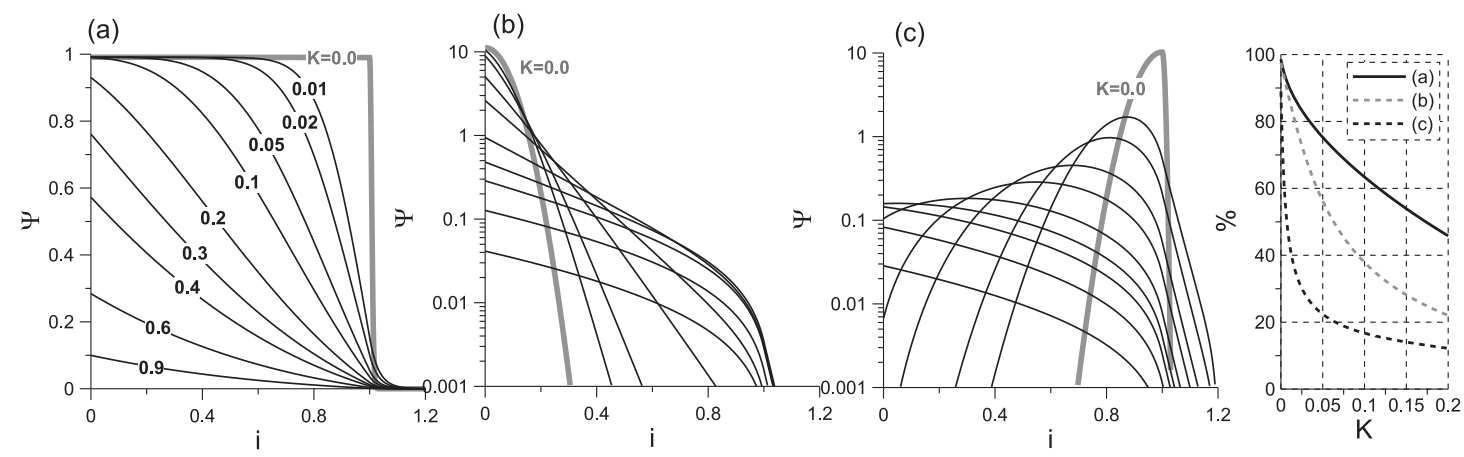

Figure 29: Three examples of the evolution of distributions of trapped particles $\Psi(i)$. All particles with $i>1$ are assumed to escape from the system within a very short time interval. The right panel shows the evolution of the number of trapped particles for these three examples. Figure is adopted from [159].

where $h=\sigma \tau h_{1}(x)$ and $h_{1}(x)$ is defined by $x(t)$ solution of Hamiltonian equation for Hamiltonian (30).

The boundary of the region filled by trapped trajectories is determined by the (changing along the trajectory) value of the area $S_{\text {res }}$. Renormalizing $i=2 \pi I_{\phi} / S_{\text {res }}$, we can rewrite Eq. (195) in a dimensionless form:

$$
\frac{\partial \Psi}{\partial K}=\frac{\partial}{\partial i}\left(i \frac{\partial \Psi}{\partial i}\right)
$$

where $K=\sigma \tau h_{1}(x) / S_{\text {res }}$. Equation (196) describes the evolution of the distribution $\Psi(i)$ of trapped particles. For a given initial distribution, one can calculate the number of particles remaining trapped for a given value of $K$. Solutions of Eq. (196) are shown in Fig. 29 for three types of initial distributions: (a) a uniformly filled region of trapped particles, (b) trapped particles originally residing only at the bottom of the potential well, and (c) for only recently trapped particles, with $i \sim 1$. The right panel of Fig. 29 shows that in all three cases the number of trapped particles diminishes as $K$ increases, ultimately decreasing to $50 \%-20 \%$ already for $K=0.2$. Consequently, to estimate the amount of detrapped (escaped) particles in realistic systems, one simply needs to evaluate $K=\sigma \tau h_{1}(x) / S_{\text {res }}$.

\subsection{Wave-particle resonance in a fluctuating magnetic field}

Let us a consider particular plasma system with magnetic field fluctuations and demonstrate the applicability of proposed approach. The geometry of the system in the same as in Subsect. 6.2: a background magnetic field $B_{0}$ and a random magnetic field $B_{\Gamma}(t)$ are directed along the $z$-axis, a plane electromagnetic wave (with a frequency $\omega$ and wave vector $k$ ) propagates along the $x$-axis, particles move in the $(x, y)$ plane. Equations of motion of a non-relativistic particle (a relativistic analog was considered in [163]) are

$$
\begin{aligned}
& \dot{x}=v_{x}, \quad \dot{y}=v_{y} \\
& \dot{v}_{x}=\Omega_{0} v_{y}\left(1+\Gamma(t)-b_{z} \sin (\phi)\right) \\
& \dot{v}_{y}=-\Omega_{0} v_{\phi} b_{z} \sin (\phi)-\Omega_{0} v_{x}\left(1+\Gamma(t)-b_{z} \sin (\phi)\right)
\end{aligned}
$$

System (197) is equivalent to system (109) with $v_{\phi}=\omega / k$ and

$$
v_{y}=-\Omega_{0}\left(x+\Gamma(t)+b_{z} k^{-1} \cos \phi\right), \quad v_{x}=p_{x} / m
$$

The wave amplitude is $B_{z}=b_{z} B_{0}$, gyrofrequency is $\Omega_{0}=e B_{0} / m c$, and magnetic field fluctuations are $B_{\Gamma}(t)=\Gamma(t) B_{0}$. We assume that $\Gamma(t)$ is a random process with zero mean (a non-zero mean can be included in $B_{0}$ ). $\Gamma(t)$ is constant during the fixed time interval $\tau$ and when $t=t_{n}=\tau n(n=1,2,3 \ldots)$ the value of $\Gamma(t)$ changes randomly according to its probability distribution. We also assume that $k \gg 1$ and the high-frequency fluctuations corresponds to $\tau \ll 1$. This means that during one cyclotron gyration a particle experiences many $\Gamma(t)$ changes. The averaging of Eq. (197) over the fast-oscillating random field corresponds to omitting the terms with $\Gamma(t)$. In such a system, the majority of particles moves around the Larmor circles described by Eqs. (100). Over one period of Larmor rotation, most of the 
particles scatter on resonance. However, some particles can be trapped into resonance. In the absence of the random field $\Gamma(t)$, the equations of trapped motion are

$$
\dot{v}_{y}=-\Omega_{0} v_{\phi}, \quad \ddot{\phi}=\Omega_{0} k v_{y}\left(1-b_{z} \sin (\phi)\right)
$$

As $k \gg 1, \phi$ changes much faster than $v_{y}$. The Hamiltonian for the second equation in (199) is

$$
H_{\phi}=\frac{1}{2} k^{2} P_{\phi}^{2}-\Omega_{0} k^{-1} v_{y}\left(\phi+b_{z} \cos (\phi)\right)
$$

where $P_{\phi}=\dot{\phi} / k^{2}$. A characteristic frequency of $\phi$ oscillations is $\Omega_{t r}=\left(\left|v_{y}\right| b_{z} k\right)^{1 / 2}$.

The trapped motion has an adiabatic invariant, $I_{\phi}=(1 / 2 \pi) \oint P_{\phi} d \phi$, which is the area under the unperturbed trajectory. The once-trapped particle stays trapped as long as $2 \pi I_{\phi}$ is smaller than the area $S_{\text {res }}$,

$$
S_{r e s}=\frac{2 \sqrt{\Omega_{0} k\left|v_{y}\right|}}{k^{2}} \int_{\phi_{-}}^{\phi_{+}} \sqrt{\phi-\phi_{+}+b_{z}\left(\cos (\phi)-\cos \left(\phi_{+}\right)\right)} d \phi
$$

where $\phi_{ \pm}$are solutions of the equation $\sin \phi=1 / b_{z}$. As the particle accelerate in the resonance, $\left|v_{y}\right| \sim v_{\phi} t$, see Eq. (199), and the value of $S_{\text {res }}$ grows. Together with the conservation of $I_{\phi}$ (which is the case in the absence of fluctuations of the magnetic field) the growth of $S_{\text {res }}$ ensures the permanent trapping.

The particle motion is qualitatively different for different values of $\Omega_{t r} \tau$. We consider the case $\Omega_{t r} \tau \ll 1$. The presence of fast oscillations of the magnetic field does not significantly change the dynamics of particles during the Larmor rotation, but, as we showed above, is important for the resonance motion. Equations (199) become

$$
\begin{aligned}
& \dot{v}_{y}=-\Omega_{0} v_{\phi}(1+\Gamma(t)) \\
& \ddot{\phi}=\Omega_{0} k v_{y}\left(1-b_{z} \sin (\phi)\right)+\Omega_{0} k v_{y} \Gamma(t)
\end{aligned}
$$

Due to random fluctuations, the value of $I_{\phi}$ changes. As $\Omega_{t r}=d H_{\phi} / d I_{\phi}$ (see [162]), we have (cf. Eq. (190)):

$$
\left(\Delta I_{\phi}\right)_{n} \approx \frac{1}{\Omega_{t r}} k^{2} P_{\phi}\left(\Delta P_{\phi}\right)_{n}=\frac{1}{\Omega_{t r}} P_{\phi} \tau \Omega_{0} k v_{y} \Gamma\left(t_{n}\right)
$$

A typical dynamics of a single particle is illustrated in Fig. 30. The particle is trapped at the rightmost point of the small circle at the center of Fig. 30(left panel). At the moment of trapping, $I_{\phi}=S_{r e s} / 2 \pi$. Then $S_{\text {res }}$ starts growing as $\sim\left(v_{\phi} \Omega_{0} b_{z} k t\right)^{1 / 2}$ and $I_{\phi}$ changes according to Eq. (203). If $\tau$ is sufficiently small, at the initial stage $S_{\text {res }}$ grows faster than $I_{\phi}$ and particle falls toward the bottom of the potential well during the first few rotations in the $\left(\phi, P_{\phi}\right)$ plane. While the particle is deep inside the well, its dynamics is quite similar to the dynamics without $\Gamma(t)$ discussed above. The particle accelerates (the horizontal strip, with the growing width in Fig. 30(left panel)) and $\Omega_{t r}$ grows. In Fig. 30(right panel), the dashed line is $\Omega_{t r}=\left(v_{\phi} \Omega b_{z} k t\right)^{1 / 2}$ and the solid line is the numerical value, obtained as the inverse of twice the time between two consecutive crossings of the $\dot{\phi}=0$ line (times $2 \pi$ ). The deviations of the numerical value from the theoretical line are due to the (weak) dependence of $\Omega_{t r}$ on $I_{\phi}(t)$, in other words, on the position of the particle inside the separatrix loop. If the particle comes close to the separatrix, the ratio $2 \pi I_{\phi}(t) / S_{\text {res }}(t)$ increases and $\Omega_{t r}(I)$ decreases, which is the most pronounced near $t \Omega_{0} \approx 68$ and at the very right, where the particle comes close to the separatrix and eventually crosses it. However, the dependence of $\Omega_{t r}$ on $I_{\phi}(t)$ is indeed quite weak: deviations of numerical $\Omega_{t r}(t)$ from theoretical curve are much smaller than variations of $I_{\phi}(t)$.

The evolution of $I_{\phi}$ can be viewed as random walk (203) with time-dependent statistics of the steps, see Fig. 30(right panel). The inset shows the magnification of a typical interval containing several periods, $\sim 2 \pi / \Omega_{t r}$. If in the process of the random walk the value of $2 \pi I_{\phi}$ exceeds the current value of $S_{\text {res }}$, the particle is released from resonance and starts moving along a larger Larmor circle.

To describe evolution of the particle probability distribution $\Psi\left(I_{\phi}, t\right)$, we consider Eq. (194) with the diffusion coefficient $D_{I I}(I, t)$ given by the variance of the right hand side of Eq. (203):

$$
D_{I I} \approx\left(\frac{k v_{y} \tau \Omega_{0}}{\Omega_{t r}}\right)^{2} \operatorname{Var}\left(P_{\phi}\left(t_{n}\right) \Gamma\left(t_{n}\right)\right)
$$



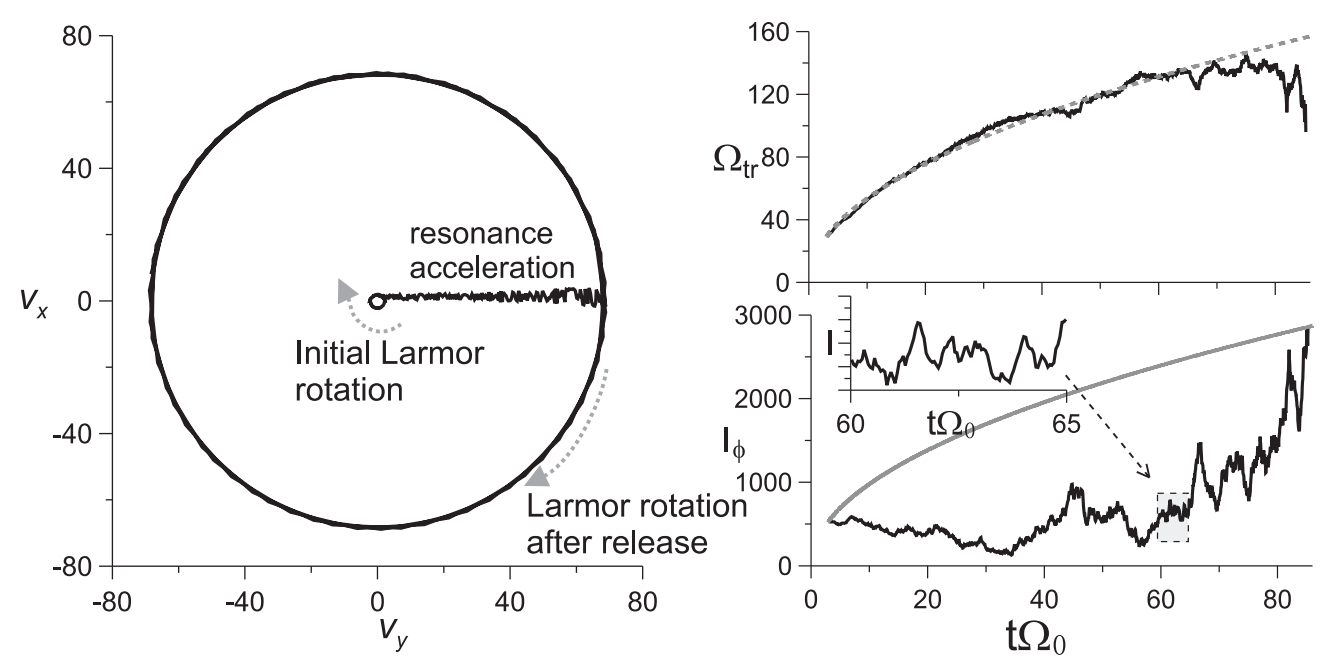

Figure 30: Capture and release in the presence of fluctuations. Figure is adopted from [158].

In Eq. (204) we took into account that random walk (203) has two distinct time scales. The first one is the period of a single rotation in resonance $\left(\sim 2 \pi / \Omega_{t r}\right)$. The other is the time at which the resonance orbit evolves. Results of numerical simulations presented in Fig. 30 indicate that a typical particle makes many turns before being released from trapping, and for a one period of trapped motion the values of $\Omega_{t r}$ and $v_{y}$ can be considered to be constant. Assuming that $P_{\phi}\left(t_{n}\right)$ and $\Gamma\left(t_{n}\right)$ are uncorrelated and have zero mean, we get (cf. Eqs. $\left.(192,193)\right)$

$$
D_{I I} \approx\left(\frac{v_{y} \tau \Omega_{0}}{\Omega_{t r}}\right)^{2} \Omega_{t r} I_{\phi} \sigma
$$

where we denoted $\sigma=\operatorname{Var}\left(\Gamma\left(t_{n}\right)\right)$. As it was noted above, we can neglect the dependence of $\Omega_{t r}$ on $I_{\phi}$ (except in the immediate vicinity of the separatrix) and assume $\Omega_{t r} \sim\left(\left|v_{y}\right| \Omega_{0} b_{z} k\right)^{1 / 2}$. As $\left|v_{y}\right| \sim v_{\phi} \Omega_{0} t$, we get

$$
\frac{\partial \Psi}{\partial t}=t^{3 / 2} D_{0} \frac{\partial}{\partial I_{\phi}}\left(I_{\phi} \frac{\partial \Psi}{\partial I_{\phi}}\right), \quad D_{0}=\Omega_{0}^{3} \sqrt{\frac{v_{\phi}^{3}}{b_{z} k}} \tau \sigma
$$

Introducing a new time, $t^{\prime}=(2 / 5) t^{5 / 2}$, we can reduce Eq. (206) to a standard diffusion equation

$$
\frac{\partial \Psi}{\partial t^{\prime}}=D_{0} \frac{\partial}{\partial I_{\phi}}\left(I_{\phi} \frac{\partial \Psi}{\partial I_{\phi}}\right)
$$

The long-time dynamics of an ensemble of particles can be described as follows: assume the initial distribution, $\Psi\left(I_{\phi}, 0\right)$, to be a $\delta$-function at a given value of $I_{\phi}=I_{0}$. The value of $S_{r e s}$ at that moment is $S_{0}=2 \pi I_{0}$. After that $S_{r e s}$ starts growing as $S_{\text {res }} \sim \sqrt{\Omega_{0} b_{z} v_{\phi} t / k^{3}} \sim\left(t^{\prime}\right)^{1 / 5}$. Meanwhile the evolution of $I_{\phi}$ can be described as a random walk (203) and $\Psi\left(I_{\phi}, t\right)$ starts drifting and spreading according to (207). It follows from (207) that on a given trajectory the expected value of $I_{\phi}$ grows as $\sim D_{0} t^{\prime} \sim D_{0} t^{5 / 2}$. In an asymptotical regime (when $t \gg 1$ ) $S_{\text {res }}$ grows slower than the expected value of $I_{\phi}$. At a certain moment $t=t_{*}$ defined as $2 \pi I_{\phi}\left(t_{*}\right)=S_{\text {res }}\left(t_{*}\right)$, the particle is released from trapping. At that moment $\sqrt{\Omega_{0}^{2} b_{z} v_{\phi} t_{*} / k^{3}} \sim 2 \pi D_{0} t_{*}^{5 / 2}$, and the velocity of a particle at the moment of release is

$$
v_{\max }=v_{\phi} \Omega_{0} t^{*} \sim v_{\phi} \sqrt{\frac{b_{z}}{2 \pi \sigma} \frac{1}{\tau k v_{\phi}}}
$$

We performed a set of numerical simulations for different values of $k, \tau$, and $\sigma$. For each set of parameters, we computed the average value of the maximum velocities achievable in the process of trapping by integrating Eq. (197) 

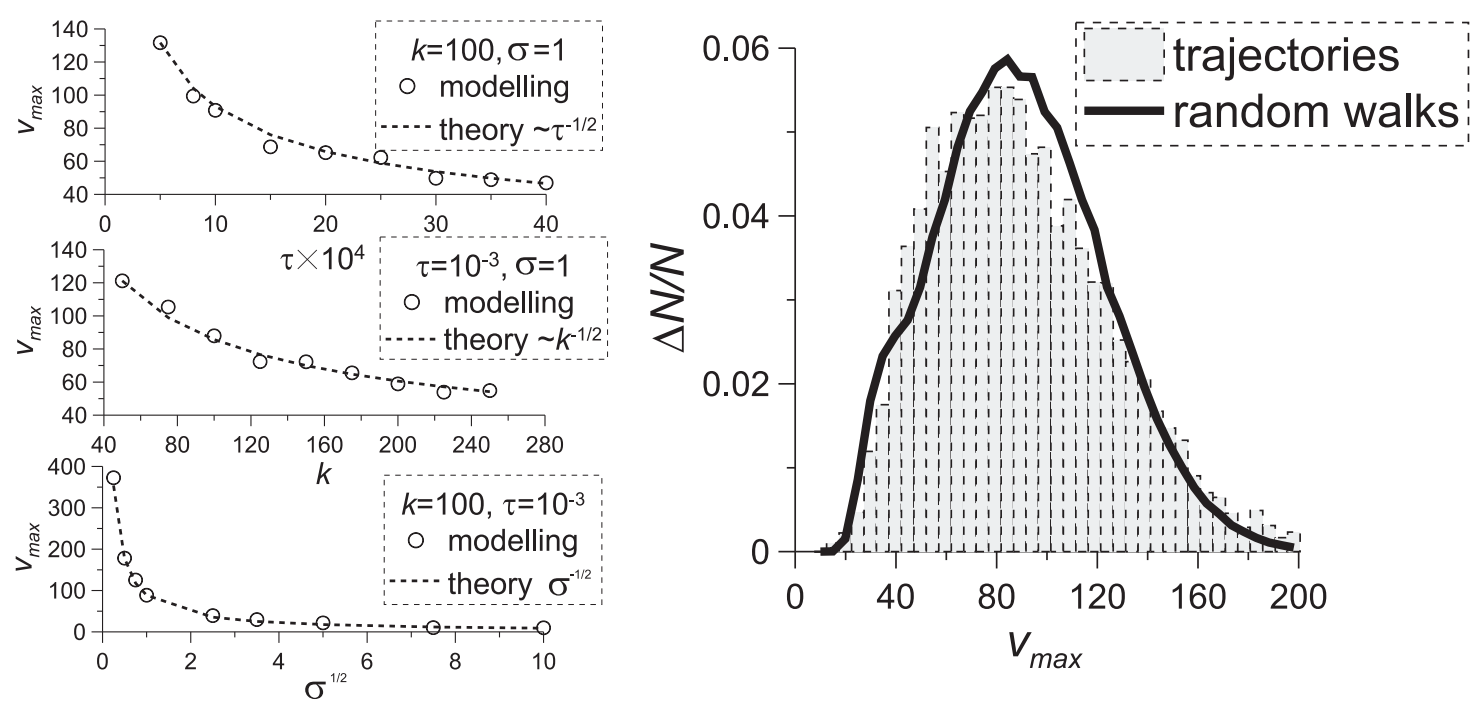

Figure 31: Ensemble average of $v_{\max }$ as a function of $k, \tau$, and $\sigma$ (left panels). The distributions of $v_{\max }$ (right panel). $\Delta N$ is the number of particles with $v_{\max }$ and $N$ is the total number of particles. Figure is adopted from [158].

for an ensemble of $10^{3}$ particles all starting with the initial energy $h_{\text {init }}=\left(v_{x}^{2}+v_{y}^{2}\right) / 2 v_{\phi}^{2}=2$ (for each particle, the value of the initial angle $\arctan \left(v_{y} / v_{x}\right)$ was chosen randomly). For each trajectory we used an individual realization of $\Gamma(t)$. We computed the final velocity and averaged it over the ensemble. The results are presented in Fig. 31(left panels). The symbols are the results of numerical simulations and the curves are obtained from analytic description (208). One can see that the estimates of scaling of $v_{\max }$ are in a good agreement with the results of the direct numerical modeling.

To verify the PDF-based description (207) and the evolution as random walk (203), we performed two types of numerical simulations. We integrated exact system (197) with $\omega / \Omega_{0}=100, \sigma=1, \tau \Omega_{0}=10^{-3}$ for $10^{4}$ particles with initial energy $h_{\text {init }}=2$ until the release from resonance and computed the distribution of $v_{\text {max }}$. We also computed $10^{6}$ trajectories as a random walk of $I_{\phi}$ using (203) as long as $I_{\phi}(t)<S_{\text {res }}(t) / 2 \pi$. For each trajectory we obtained its value $t_{*}$ such that $I_{\phi}\left(t_{*}\right)=S_{\text {res }}\left(t_{*}\right) / 2 \pi$ and computed $v_{\max }=v_{\phi} \Omega_{0} t_{*}$. Two distributions of $v_{\max }$ are presented in Fig. 31(right panel): their similarity shows that the random walk approximation can adequately describe the dynamics of Eq. (197).

Section summary. In this section we described effects of the background magnetic field fluctuations on wave-particle resonant interaction. We showed that resonant interaction can be destroyed by fluctuations and trapped particles could escape from resonance due to external nonresonant fluctuations. This effect imposes a limit on resonant particle acceleration in realistic space plasma environments.

\section{Discussion and open questions}

\subsection{Four steps to kinetic equation}

The theory of nonlinear resonant wave-particle interaction started from pioneering works of [164, 34, 35] and rapidly developed into a significant branch of plasma physics [e.g., 165, 166]. In last two decades this theory became increasingly important for investigations of space and laboratory plasma systems as advanced measurements of wave signal onboard modern spacecraft $[167,168,60,169,170,171,172,173]$ and in laboratory experiments [93, 94, $95,96]$ pointed at a significantly nonlinear nature of charged particle interaction with many different plasma waves. Although the quasi-linear theory of particle scattering by broad ensembles of low amplitude waves still provides quite a satisfactory explanation of many observed features of the long-term dynamics in various near-Earth plasma systems [e.g., 174, 87], more and more often the theory of nonlinear resonances is required to properly describe transient variations of measured charged particle fluxes [e.g., 175, 176].

In this review we considered a method construction of universal approach for description of the particle distribution evolution in systems with numerous nonlinear resonances. This approach consists of four main steps 
Averaging and structure of resonance: Starting from the Hamiltonian for resonant particles in a specific electromagnetic field configuration, obtain expressions for Hamiltonians of slow motion and determine the location of resonance(s) in the phase plane;

Individual resonance interaction: Determine the properties of a single resonant interaction (the amplitude of scattering, energy change due to trapping) for a individual particle trajectory;

Collective properties at multiple crossings: Average the individual resonant particle properties over a particle ensemble and multiple resonance interactions to determine collective properties: probability of trapping, velocity of particle drift in energy space, energy drift and diffusion due to scattering;

Kinetic equation: Construct a kinetic equation including collective (averaged) properties of resonant interaction;

A kinetic (master) equation derived following these steps is [177]

$$
\frac{\partial \Psi}{\partial t}=\int K\left(\mathbf{v}-\mathbf{v}^{\prime}\right) \Psi\left(\mathbf{v}^{\prime}\right) d \mathbf{v}^{\prime}
$$

with an analytical expression for operator $K\left(\mathbf{v}, \mathbf{v}^{\prime}\right)$. The derived kinetic equation opens an opportunity to quantify the impact of nonlinear wave-particle interaction on dynamics of particles in various space plasma systems. Although this equation is rather general, there are several questions which should be addressed before for realistic plasma systems.

\subsection{Open questions}

So far the evolution of particle distributions, $\Psi$, in the system with the nonlinear wave-particle interaction was described using a simplified test particle approach [e.g., 178, 179] or by a numerical construction of an integral operator $K$ acting on the particle distribution $[180,181]$. These approaches, when tailored to a particular parameter range, can provide a sufficiently accurate description of the resonant particle dynamics in some plasma systems. However, their accuracy is not sufficient for a description of long-term evolution of $\Psi$ due to very different scales of resonant particle velocity change in two nonlinear phenomena: scattering and trapping. A small population of particles is trapped and accelerated by intense waves, and an accurate numerical modelling of this population must take a large range of velocities into account. On the other hand, the vast majority of particles are scattered and change their velocities only slightly. Simultaneous consideration of both effects presents a significant challenge for any numerical approach. Moreover, application of numerical approach does not allow controlling the fine relation between scattering and trapping energy (velocity) change, and thus the corresponding numerical results can deviate from the actual $\Psi$ evolution on long time-scales.

The alternative approach for derivation of Eq. (209), that was demonstrated in Sect. 5.3, results in a kinetic equation where trapping and scattering effects are balanced. This approach requires a detailed information about the wave-particle nonlinear interactions, such as the probability of trapping and scattering amplitude. In this review we collected results from several of our previous studies to propose a standardized approach. Following this procedure, one can apply the same technique for different configuration of background magnetic field and different wave properties and derive an appropriate kinetic equation for $\Psi$. However, we should mention that the approach described in Sect. 5.3 is not yet finalized. Several important questions remain open:

1. How to generalize Eq. (96) to systems with several frequencies of slow particle motion (i.e., multidimensional systems)?

2. How to include the averaging of the coefficients Eq. (96) over distributions of wave characteristics (e.g., frequency, intensity, etc.)?

3. How to apply Eq. (96) to systems with nonharmonic waves (e.g., localized wave packets)?

4. How to include effects of the resonant interaction destruction due to external resonant and nonresonant noises into Eq. (96)?

5. What are general properties of solutions of Eq. (96)? 
Some of these questions are considered in recent publications [182, 183, 184], but so far no one of them is solved.

In this review we focused on effects of the nonlinear resonant wave-particle interaction on particle dynamics, and did not discuss the feedback of this dynamics to wave evolution. This approximation is based on the assumption that the acceleration/deceleration of some population of resonant particles occurs at expense of deceleration/acceleration of another resonant population [22, 23]. However, for closed plasma systems where the energy exchange between particle populations is not influenced by external energy sources, Eq. (96) should be written for all resonant particles and be supplemented by the second equation for the average wave intensity [like it has been done for quasi-linear models, see, e.g., 166].

\section{Conclusions}

In this review we presented a general approach for analysis of Hamiltonian systems with resonances and discussed several concrete examples where this approach allows us to explain charged particle dynamics and acceleration. We mostly restricted our consideration to dynamics of individual particles and only sketched the construction of general kinetic equations where effects of particle trapping and phase bunching (nonlinear particle scattering) are included in a form of operators acting on particle distribution function. Currently, the analysis of individual particle trajectories can still provide useful information of many plasma system with nonlinear wave-particle interaction. However, the natural next step in the development of this theory be a construction of a universal kinetic equation describing fast particle transport in the phase space due to trapping. This equation should be eventually complemented by equations for wave intensity evolution due to the feedback. Although some approaches for the construction of this self-consistent system of equations can be derived from our review, there are a lot to be done to finalize the kinetic theory of the nonlinear wave-particle interaction.

\section{Acknowledgements}

Work of A.V.A, A.I.N., A.A.V., and I.Y.V. is supported by the Russian Scientific Foundation, Project No. 1412-00824. This material is based in part upon work supported by the National Science Foundation under Award No. CMMI-1362782 (D.L.V.).

\section{Appendix A. Conservation of the adiabatic invariant $I_{\phi}$ and accuracy of integration}

In Sect 3 we demonstrated that description of trapped particle motion is based on the conservation of adiabatic invariant $I_{\phi}$ given by Eq. (36). Relation between this invariant and the area $S_{\text {res }}$ surrounded by the separatrix determines for how long do trapped particles stay in the resonance before the escape (see scheme in Fig. 5). Therefore, the accuracy of numerical integration of Hamiltonian equations should be sufficient to assure the $I_{\phi}$ conservation. Equations of motions contain terms of the different order of small parameter $\varepsilon$. To show what terms are important for $I_{\phi}$ conservation (see, e.g., the discussion in [185] and references therein), we consider the accuracy of integration of Hamiltonian equations for systems with two pairs of variables and a time-dependent perturbation (e.g., 171).

For the sake of simplicity, we start with a Hamiltonian in a dimensionless form:

$$
H=H_{0}\left(z, p_{z}, I_{x}\right)+\varepsilon u\left(z, p_{z}, I_{x}\right) \sin (\phi-n \psi), \phi=\int k(\tilde{z}) d \tilde{z}-\omega t
$$

where $\varepsilon \ll 1$, pairs of conjugate variables are $\left(z, p_{z}\right)$ and $\left(\psi, I_{x}\right)$; the order of resonance $n=0$ (for system (157)) and $n=-1$ (for system (171)). The corresponding Hamiltonian equation are

$$
\begin{aligned}
\dot{z} & =\frac{\partial H_{0}}{\partial p_{z}}+\varepsilon \frac{\partial u}{\partial p_{z}} \sin (\phi-n \psi) \\
\dot{p}_{z} & =-\frac{\partial H_{0}}{\partial z}+k \varepsilon u \cos (\phi-n \psi)+\varepsilon \frac{\partial u}{\partial z} \sin (\phi-n \psi) \\
\dot{\psi} & =\frac{\partial H_{0}}{\partial I_{x}}+\varepsilon \frac{\partial u}{\partial I_{x}} \sin (\phi-n \psi) \\
\dot{I}_{x} & =-n \varepsilon u \cos (\phi-n \psi)
\end{aligned}
$$


These equations contain terms independent on $\varepsilon$, terms proportional to $k \varepsilon$, and terms proportional to $\varepsilon$. The later terms are small compared to other terms. However, these small terms are important for accurate system expansion around some resonance. To demonstrate situations when terms $\sim \varepsilon$ should be taken into account, we consider the resonance $\dot{\phi}-n \dot{\psi}=0$. First, we introduce new phase $\zeta=\phi-n \psi$ and conjugate momentum $I$ through the generating function:

$$
R=I\left(\int k(\tilde{z}) d \tilde{z}-\omega t-n \psi\right)+P_{z} z+\tilde{I}_{x} \psi
$$

New variables are:

$$
p_{z}=k I+P_{z}, \quad I_{x}=-n I+\tilde{I}_{x}, \quad \zeta=\int k(\tilde{z}) d \tilde{z}-\omega t-n \psi
$$

The corresponding Hamiltonian takes the form:

$$
H=H_{0}\left(z, k I+P_{z},-n I+\tilde{I}_{x}\right)+\varepsilon u\left(z, k I+P_{z},-n I+\tilde{I}_{x}\right) \sin \zeta
$$

where conjugate variables are $\left(z, P_{z}\right),(\zeta, I)$, and $\left(\psi, \tilde{I}_{x}\right)$. Hamiltonian (A.5) does not depend on $\psi$, and thus $\tilde{I}_{x}$ is conserved. We can set $\tilde{I}_{x}=0$ and rewrite Eq. (A.5) as:

$$
H=H_{0}\left(z, k I+P_{z},-n I\right)+\varepsilon u\left(z, k I+P_{z},-n I\right) \sin \zeta
$$

The resonance condition is $\dot{\zeta}=\partial H_{0} / \partial I=0$ with a solution $I=I_{\text {res }}\left(z, P_{z}\right)$. Expanding Hamiltonian (A.6) around $I-I_{\text {res }}=0$ we get:

$$
\begin{aligned}
& H=\Lambda+\frac{1}{2} g\left(I-I_{\text {res }}\right)^{2}+\varepsilon u_{\text {res }} \sin \zeta \\
& \Lambda\left(z, P_{z}\right)=H_{0}\left(z, k I_{\text {res }}+P_{z},-n I_{\text {res }}\right) \\
& g\left(z, P_{z}\right)=\partial^{2} H_{0} /\left.\partial I^{2}\right|_{I_{\text {res }}}, \quad u_{\text {res }}\left(z, P_{z}\right)=u\left(z, k I_{\text {res }}+P_{z},-n I_{\text {res }}\right)
\end{aligned}
$$

Introducing new variable $P_{\phi}=\left(I-I_{\text {res }}\right)$, we obtain:

$$
H=\Lambda+\frac{1}{2} g P_{\phi}^{2}+r \zeta+\varepsilon u_{r e s} \sin \zeta, \quad r\left(z, P_{z}\right)=\left\{\Lambda, I_{r e s}\right\}
$$

Hamiltonian equation for $P_{\phi}$ are

$$
\dot{P}_{\phi}=\frac{\partial H}{\partial \zeta}=-r-\varepsilon u_{r e s} \cos \zeta
$$

Variables $r, u_{\text {res }}$ change slowly due to a (slow) variation of $\left(z, P_{z}\right)$. Equation (A.9) shows that the accuracy of conservation of $I_{\phi}=\oint P_{\phi} d \phi$ is defined by the accuracy of calculation of $r$ and $u_{r e s}$. Both functions depend on $I_{r e s}$, which is defined by the equation $\dot{\zeta} \partial H_{0} / \partial I=0$. We rewrite this equation through the initial variables $p_{z}, I_{x}$. For this reason, we take into account that $\partial I / \partial p_{z}=1 / k$ and $\partial I / \partial I_{x}=-n$ (see Eq. (A.4)). Thus, $\partial H_{0} / \partial I=0$ takes the form

$$
k \frac{\partial H_{0}}{\partial p_{z}}-n \frac{\partial H_{0}}{\partial I_{x}}=0
$$

We substitute Eqs. (A.2) to Eq. (A.10):

$$
\frac{\partial H_{0}}{\partial I}=k\left(\dot{z}-\varepsilon \frac{\partial u}{\partial p_{z}} \sin (\phi-n \psi)\right)-n\left(\dot{\psi}-\varepsilon \frac{\partial u}{\partial I_{x}} \sin (\phi-n \psi)\right)=0
$$

Equation (A.11) does not contain terms $\sim \varepsilon$ only if $n=0$ (the Landau resonance, see, e.g., system (157)) and $\partial u / \partial p_{z}=0$. In this case, to find $I_{r e s}$ we do not need to take into account the second order term $\sim \varepsilon$ in system (A.2).

For $n \neq 0$ and/or $\partial u / \partial p_{z} \neq 0$, the accuracy of computation of $I_{r e s}$ depends on terms $\sim \varepsilon$ and all these terms should be taken into account for a numerical integration of initial system (A.2). This simple example shows that for numerical integration of system (A.2), one should keep all small terms and provide the corresponding accuracy of integration. 


\section{Appendix B. Accuracy of averaging of particle gyrorotation}

Through the review, we consider many systems where particles oscillate around a strong background magnetic field. When considering the resonant wave-particle interaction, these fast oscillations should be averaged over. Such an averaging is equivalent to the introduction of a new adiabatic invariant. We use a canonical transformation to perform the averaging. However, for systems with a weakly inhomogeneous background magnetic field, this transformation results in an appearance of new fast oscillating terms in the Hamiltonian equations. In this Appendix we derive expressions for these terms and explain why they can be omitted.

We use a nonrelativistic Hamiltonian, but the same procedure can be performed for relativistic systems:

$$
H=\frac{1}{2 m}\left(p_{x}^{2}+p_{z}^{2}\right)+\frac{1}{2} m\left(\Omega_{0}(z) x\right)^{2}
$$

where $\Omega_{0}(z)=e B_{0}(z) / m c$ is a gyrofrequency. In systems with a strong magnetic field, particle gyromotion in the $\left(x, p_{x}\right)$ plane is much faster than the motion along field lines in the $\left(z, p_{z}\right)$ plane. This approximation is called the approximation of a weak magnetic field inhomogeneity as it is assumed that $x \partial \Omega_{0} / \partial z \ll \Omega_{0}$. Hamiltonian equations for $\left(x, p_{x}\right)$ are:

$$
\dot{x}=p_{x} / m, \quad \dot{p}_{x}=-m \Omega_{0}^{2}(z) x
$$

For frozen $\left(z, p_{z}\right)$, a solution of Eqs. (B.2) is:

$$
x=\sqrt{\frac{2 h_{z}}{m \Omega_{0}^{2}}} \sin \psi, \quad p_{x}=\sqrt{2 h_{z} m} \cos \psi
$$

where $h_{z}=H-p_{z}^{2} / 2 m$ and $\dot{\psi}=\Omega_{0}$. The corresponding action $I_{x}$ can be written as

$$
I_{x}=\frac{1}{2 \pi} \oint p_{x} d x=\frac{2 h_{z}}{\pi \Omega_{0}} \int_{0}^{\pi} \cos ^{2} \psi d \psi=\frac{h_{z}}{\Omega_{0}}
$$

Thus, Eq. (B.3) can be rewritten as

$$
x=\sqrt{\frac{2 I_{x}}{m \Omega_{0}}} \sin \psi, \quad p_{x}=\sqrt{2 I_{x} \Omega_{0} m} \cos \psi
$$

We introduce $\left(\psi, I_{x}\right)$ as new canonical variables using the generating function:

$$
\begin{aligned}
R\left(I_{x}, x ; \tilde{p}_{z}, z\right) & =\int p_{x} d x+\tilde{p}_{z} z=2 I_{x} \int \cos ^{2} \psi d \psi+\tilde{p}_{z} z=I_{x}(\psi+\sin \psi \cos \psi)+\tilde{p}_{z} z \\
& =I_{x}\left(\arcsin \left(x \sqrt{\frac{m \Omega_{0}}{2 I_{x}}}\right)+x \sqrt{\frac{m \Omega_{0}}{2 I_{x}}} \sqrt{1-x^{2} \frac{m \Omega_{0}}{2 I_{x}}}\right)+\tilde{p}_{z} z
\end{aligned}
$$

The new and old variables are related as

$$
\begin{aligned}
& p_{x}=\frac{\partial R}{\partial x}=2 I_{x} \sqrt{\frac{m \Omega_{0}}{2 I_{x}}} \sqrt{1-x^{2} \frac{m \Omega_{0}}{2 I_{x}}}=\sqrt{2 I_{x} m \Omega_{0}} \cos \psi \\
& p_{z}=\frac{\partial R}{\partial z}=\tilde{p}_{z}+\frac{I_{x}}{\Omega_{0}} \frac{\partial \Omega_{0}}{\partial z} x \sqrt{\frac{m \Omega_{0}}{2 I_{x}}} \sqrt{1-x^{2} \frac{m \Omega_{0}}{2 I_{x}}}=\tilde{p}_{z}+I_{x} \frac{1}{2} \frac{\partial \ln \Omega_{0}}{\partial z} \sin 2 \psi
\end{aligned}
$$

and $\tilde{z}=z$. In the new variables the Hamiltonian is

$$
\begin{aligned}
\tilde{H} & =\frac{1}{2 m}\left(\tilde{p}_{z}+I_{x} \frac{1}{2} \frac{\partial \ln \Omega_{0}}{\partial z} \sin 2 \psi\right)^{2}+I_{x} \Omega_{0} \\
& =\frac{1}{2 m} \tilde{p}_{z}^{2}+I_{x} \Omega_{0}+\tilde{p}_{z} I_{x} \frac{\partial \ln \Omega_{0}}{\partial z} \sin 2 \psi+\left(I_{x} \frac{1}{2} \frac{\partial \ln \Omega_{0}}{\partial z}\right)^{2} \sin ^{2} 2 \psi
\end{aligned}
$$


There are two fast oscillating terms in (B.8): $\sim \sin 2 \psi$ and $\sim \sin ^{2} 2 \psi$. Both terms result in oscillations of adiabatic invariant $I_{x}$, as $\dot{I}_{x}=-\partial \tilde{H} / \partial \psi$. Let us consider effects of these two terms separately.

The term $\sim\left(\partial \ln \Omega_{0} / \partial z\right) \sin 2 \psi$ is small (because the inhomogeneity of the background magnetic field is weak) and oscillates fast around zero. Thus, instead of $I_{x}$ we can introduce an improved adiabatic invariant and this procedure removes term $\sim\left(\partial \ln \Omega_{0} / \partial z\right) \sin 2 \psi$ from the Hamiltonian [104]. The amplitude of the $\sin ^{2} 2 \psi$ term in Hamiltonian (B.8) is $\sim\left(\partial \ln \Omega_{0} / \partial z\right)^{2}$ and much smaller than other terms again because the inhomogeneity of the background magnetic field is weak. For systems with and without wave perturbations this term can be omitted. Therefore, we can rewrite Hamiltonian (B.8) as

$$
\tilde{H}=\frac{1}{2 m} \tilde{p}_{z}^{2}+I_{x} \Omega_{0}(z)
$$

where $\tilde{p}_{z}=p_{z}$.

\section{References}

[1] L. P. Pitaevskii, E. M. Lifshitz, Vol. 10: Physical Kinetics, Course of Theoretical Physics, New York: Pergamon Press, 1981.

[2] A. A. Galeev, R. N. Sudan (Eds.), Basic Plasma Physics, 1983.

[3] A. A. Galeev, R. N. Sudan, Handbook of plasma physics. Vol. 2: Basic plasma physics II., 1985.

[4] A. A. Vedenov, Theory of a Weakly Turbulent Plasma, Reviews of Plasma Physics 3 (1967) 229.

[5] A. A. Vedenov, E. Velikhov, R. Sagdeev, Quasilinear theory of plasma oscillations, Nuclear Fusion Suppl. 2 (1962) 465475.

[6] W. E. Drummond, D. Pines, Nonlinear stability of plasma oscillations, Nuclear Fusion Suppl. 3 (1962) 1049-1058.

[7] A. A. Andronov, V. Y. Trakhtengerts, Kinetic instability of the Earths outer radiation belt, Geomagnetism and Aeronomy 4 (1964) $233-242$.

[8] C. F. Kennel, F. Engelmann, Velocity Space Diffusion from Weak Plasma Turbulence in a Magnetic Field, Physics of Fluids 9 (1966) 2377-2388. doi:10.1063/1.1761629.

[9] I. Lerche, Quasilinear Theory of Resonant Diffusion in a Magneto-Active, Relativistic Plasma, Physics of Fluids 11.

[10] V. I. Karpman, Nonlinear Effects in the ELF Waves Propagating along the Magnetic Field in the Magnetosphere, Space Sci. Rev.16 (1974) 361-388. doi:10.1007/BF00171564.

[11] D. Le Queau, A. Roux, Quasi-monochromatic wave-particle interactions in magnetospheric plasmas, Solar Phys.111 (1987) 59-80. doi:10.1007/BF00145441.

[12] J. M. Albert, Cyclotron resonance in an inhomogeneous magnetic field, Physics of Fluids B 5 (1993) 2744-2750. doi:10.1063/1.860715.

[13] V. V. Solovev, D. R. Shkliar, Particle heating by a low-amplitude wave in an inhomogeneous magnetoplasma, Sov. Phys. JETP 63 (1986) 272-277.

[14] J. M. Albert, Comparison of pitch angle diffusion by turbulent and monochromatic whistler waves, J. Geophys. Res.106 (2001) $8477-8482$. doi:10.1029/2000JA000304.

[15] A. L. Brinca, Turbulence effects in the cyclotron resonance of monochromatic whistlers, Geophys. Res. Lett.5 (1978) $839-842$. doi:10.1029/GL005i010p00839.

[16] A. L. Brinca, On the evolution of the geomagnetospheric coherent cyclotron resonance in the midst of noise, J. Geophys. Res.85 (1980) 4711-4714. doi:10.1029/JA085iA09p04711.

[17] R. L. Dowden, Detrapping by an additional wave of wave-trapped electrons, J. Geophys. Res.87 (1982) $6237-6242$. doi:10.1029/JA087iA08p06237.

[18] D. Nunn, A nonlinear theory of sideband stability in ducted whistler mode waves, Planatary Space Science34 (1986) $429-451$. doi:10.1016/0032-0633(86)90032-2.

[19] M. Schulz, L. J. Lanzerotti, Particle diffusion in the radiation belts, Springer, New York, 1974.

[20] L. R. Lyons, D. J. Williams, Quantitative aspects of magnetospheric physics., 1984.

[21] V. Y. Trakhtengerts, M. J. Rycroft, Whistler and Alfvén Mode Cyclotron Masers in Space, Cambridge University Press, 2008.

[22] D. Shklyar, H. Matsumoto, Oblique Whistler-Mode Waves in the Inhomogeneous Magnetospheric Plasma: Resonant Interactions with Energetic Charged Particles, Surveys in Geophysics 30 (2009) 55-104. doi:10.1007/s10712-009-9061-7.

[23] D. R. Shklyar, On the nature of particle energization via resonant wave-particle interaction in the inhomogeneous magnetospheric plasma, Annales Geophysicae 29 (2011) 1179-1188. doi:10.5194/angeo-29-1179-2011.

[24] Y. Omura, M. Hikishima, Y. Katoh, D. Summers, S. Yagitani, Nonlinear mechanisms of lower-band and upper-band VLF chorus emissions in the magnetosphere, J. Geophys. Res.114 (2009) 7217. doi:10.1029/2009JA014206.

[25] Y. Omura, D. Nunn, D. Summers, Generation Processes of Whistler Mode Chorus Emissions: Current Status of Nonlinear Wave Growth Theory, in: D. Summers, I. U. Mann, D. N. Baker, M. Schulz (Eds.), Dynamics of the Earth's Radiation Belts and Inner Magnetosphere, American Geophysical Union, 2013, pp. 243-254. doi:10.1029/2012GM001347.

[26] D. R. Shklyar, Energy transfer from lower energy to higher-energy electrons mediated by whistler waves in the radiation belts, J. Geophys. Res.122 (1) (2017) 640-655. doi:10.1002/2016JA023263. URL http://dx.doi.org/10.1002/2016JA023263

[27] A. G. Demekhov, U. Taubenschuss, O. Santolk, Simulation of vlf chorus emissions in the magnetosphere and comparison with themis spacecraft data, J. Geophys. Res.(2017) n/a-n/adoi:10.1002/2016JA023057. URL http: //dx.doi.org/10.1002/2016JA023057

[28] D. Bénisti, Nonlocal adiabatic theory. I. The action distribution function, Physics of Plasmas 24 (9) (2017) 092120. arXiv:1706.03540, doi:10.1063/1.4996957. 
[29] D. Bénisti, Nonlocal adiabatic theory. II. Nonlinear frequency shift on an electron plasma wave in a multidimensional inhomogeneous plasma, Physics of Plasmas 24 (9) (2017) 092121. doi:10.1063/1.4996963.

[30] V. L. Krasovsky, Trapped particle effect on the velocity of circularly polarized electromagnetic waves in an isotropic plasma, Physics Letters A 374 (2010) 1751-1754. doi:10.1016/j.physleta.2010.02.030.

[31] I. Y. Dodin, N. J. Fisch, Nonlinear Dispersion of Stationary Waves in Collisionless Plasmas, Physical Review Letters 107 (3) (2011) 035005. arXiv:1105.0196, doi:10.1103/PhysRevLett.107.035005.

[32] I. Y. Dodin, N. J. Fisch, Adiabatic nonlinear waves with trapped particles. II. Wave dispersion, Physics of Plasmas 19 (1) (2012) 012103. arXiv:1107.3074, doi:10.1063/1.3662115.

[33] X. Tao, F. Zonca, L. Chen, Identify the nonlinear wave-particle interaction regime in rising tone chorus generation, Geophys. Res. Lett.44 (8) (2017) 3441-3446. doi:10.1002/2017GL072624. URL http: //dx.doi .org/10.1002/2017GL072624

[34] T. O'Neil, Collisionless Damping of Nonlinear Plasma Oscillations, Physics of Fluids 8 (1965) 2255-2262. doi:10.1063/1.1761193.

[35] R. K. Mazitov, Damping of plasma waves, Journal of Applied Mechanics and Technical Physics 6 (1965) 22-25. doi:10.1007/BF00914365.

[36] L. D. Landau, E. M. Lifshitz, Vol. 8: Electrodynamics of Continuous Media, Course of Theoretical Physics, Pergamon Press, 1960.

[37] S. P. Gary, D. Montgomery, D. W. Swift, Particle acceleration by electrostatic waves with spatially varying phase velocities, J. Geophys. Res.73 (1968) 7524-7525. doi:10.1029/JA073i023p07524.

[38] G. Laval, R. Pellat, Particle acceleration by electrostatic waves propagating in an inhomogeneous plasma, J. Geophys. Res.75 (1970) 3255-3256. doi:10.1029/JA075i016p03255.

[39] V. I. Karpman, D. R. Shklyar, Nonlinear Damping of Potential Monochromatic Waves in an Inhomogeneous Plasma, Sov. JETP 35 (1972) 500 .

[40] V. I. Karpman, J. N. Istomin, D. R. Shklyar, Particle acceleration by a non-linear langmuir wave in an inhomogeneous plasma, Physics Letters A 53 (1975) 101-102. doi:10.1016/0375-9601(75)90364-3.

[41] R. Z. Sagdeev, Reviews of Plasma Physics, 1st Edition, Vol. 4, Consultants Bureau, New York, 1966.

[42] R. Z. Sagdeev, V. D. Shapiro, Influence of transverse magnetic field on landau damping, Soviet Journal of Experimental and Theoretical Physics Letters 17 (1973) 279-282.

[43] V. M. Gubchenko, V. V. Zaitsev, On proton and electron acceleration by shock waves during large solar flares, Solar Physics 63 (1979) 337-352. doi:10.1007/BF00174539.

[44] T. Katsouleas, J. M. Dawson, Unlimited electron acceleration in laser-driven plasma waves, Physical Review Letters 51 (1983) $392-395$. doi:10.1103/PhysRevLett.51.846.2.

[45] D. Nunn, Wave-particle interactions in electrostatic waves in an inhomogeneous medium, Journal of Plasma Physics 6 (1971) 291. doi: $10.1017 / \mathrm{S} 0022377800006061$.

[46] D. Nunn, A self-consistent theory of triggered VLF emissions, Planatary Space Science22 (1974) 349-378. doi:10.1016/00320633(74)90070-1.

[47] V. I. Karpman, J. N. Istomin, D. R. Shklyar, Nonlinear theory of a quasi-monochromatic whistler mode packet in inhomogeneous plasma, Plasma Physics 16 (1974) 685-703. doi:10.1088/0032-1028/16/8/001.

[48] M. L. Goldstein, D. A. Roberts, W. H. Matthaeus, Magnetohydrodynamic Turbulence In The Solar Wind, ARA\&A33 (1995) $283-326$. doi:10.1146/annurev.aa.33.090195.001435.

[49] S. P. Gary, C. W. Smith, Short-wavelength turbulence in the solar wind: Linear theory of whistler and kinetic Alfvén fluctuations, J. Geophys. Res.114 (2009) A12105. doi:10.1029/2009JA014525.

[50] J. S. Zhao, Y. Voitenko, D. J. Wu, J. De Keyser, Nonlinear Generation of Kinetic-scale Waves by Magnetohydrodynamic Alfvén Waves and Nonlocal Spectral Transport in the Solar Wind, Astrophys. J.785 (2014) 139. doi:10.1088/0004-637X/785/2/139.

[51] M. Neugebauer, Comment on the abundances of rotational and tangential discontinuities in the solar wind, J. Geophys. Res.111 (2006) A04103. doi:10.1029/2005JA011497.

[52] A. Greco, S. Perri, S. Servidio, E. Yordanova, P. Veltri, The Complex Structure of Magnetic Field Discontinuities in the Turbulent Solar Wind, Astrophys. J. Lett.823 (2016) L39. arXiv:1511.03084, doi:10.3847/2041-8205/823/2/L39.

[53] J. J. Podesta, The most intense current sheets in the high-speed solar wind near 1 AU, J. Geophys. Res.122 (2017) $2795-2823$. doi:10.1002/2016JA023629.

[54] M. V. Medvedev, V. I. Shevchenko, P. H. Diamond, V. L. Galinsky, Fluid models for kinetic effects on coherent nonlinear Alfvén waves. II. Numerical solutions, Physics of Plasmas 4 (1997) 1257-1285. arXiv:physics/9612018, doi:10.1063/1.872356.

[55] G. G. Howes, The Dynamical Generation of Current Sheets in Astrophysical Plasma Turbulence, Astrophys. J. Lett.827 (2016) L28. arXiv:1607.07465, doi:10.3847/2041-8205/827/2/L28.

[56] R. E. Ergun, D. M. Malaspina, I. H. Cairns, M. V. Goldman, D. L. Newman, P. A. Robinson, S. Eriksson, J. L. Bougeret, C. Briand, S. D. Bale, C. A. Cattell, P. J. Kellogg, M. L. Kaiser, Eigenmode Structure in Solar-Wind Langmuir Waves, Physical Review Letters 101 (5) (2008) 051101. doi:10.1103/PhysRevLett.101.051101.

[57] D. M. Malaspina, D. L. Newman, L. B. Wilson, III, K. Goetz, P. J. Kellogg, K. Kersten, Electrostatic Solitary Waves in the Solar Wind: Evidence for Instability at Solar Wind Current Sheets, J. Geophys. Res.118 (2013) 591-599. doi:10.1002/jgra.50102.

[58] C. Krafft, A. S. Volokitin, V. V. Krasnoselskikh, Interaction of Energetic Particles with Waves in Strongly Inhomogeneous Solar Wind Plasmas, Astrophys. J.778 (2013) 111. doi:10.1088/0004-637X/778/2/111.

[59] C. Krafft, A. S. Volokitin, V. V. Krasnoselskikh, Langmuir Wave Decay in Inhomogeneous Solar Wind Plasmas: Simulation Results, Astrophys. J.809 (2015) 176. doi:10.1088/0004-637X/809/2/176.

[60] S. D. Bale, P. J. Kellogg, D. E. Larsen, R. P. Lin, K. Goetz, R. P. Lepping, Bipolar electrostatic structures in the shock transition region: Evidence of electron phase space holes, Geophys. Res. Lett.25 (1998) 2929-2932. doi:10.1029/98GL02111.

[61] M. A. Balikhin, T. D. de Wit, H. S. C. K. Alleyne, L. J. C. Woolliscroft, S. N. Walker, V. Krasnosel'skikh, W. A. C. Mier-Jedrzejeowicz, W. Baumjohann, Experimental determination of the dispersion of waves observed upstream of a quasi-perpendicular shock, Geophys. Res. Lett.24 (1997) 787-790. doi:10.1029/97GL00671. 
[62] L. B. Wilson, A. Koval, A. Szabo, A. Breneman, C. A. Cattell, K. Goetz, P. J. Kellogg, K. Kersten, J. C. Kasper, B. A. Maruca, M. Pulupa, Electromagnetic waves and electron anisotropies downstream of supercritical interplanetary shocks, J. Geophys. Res.118 (2013) 5-16. arXiv:1207.6429, doi:10.1029/2012JA018167.

[63] Y. Kuramitsu, V. Krasnoselskikh, Gyroresonant Surfing Acceleration, Physical Review Letters 94 (3) (2005) 031102-+. doi:10.1103/PhysRevLett.94.031102.

[64] A. V. Artemyev, O. V. Agapitov, V. V. Krasnoselskikh, Cyclotron resonance in plasma flow, Physics of Plasmas 20 (12) (2013) 124502. doi:10.1063/1.4853615.

[65] A. Kis, O. Agapitov, V. Krasnoselskikh, Y. V. Khotyaintsev, I. Dandouras, I. Lemperger, V. Wesztergom, Gyrosurfing Acceleration of Ions in Front of Earth's Quasi-parallel Bow Shock, Astrophys. J.771 (2013) 4. doi:10.1088/0004-637X/771/1/4

[66] L. B. Wilson, D. G. Sibeck, D. L. Turner, A. Osmane, D. Caprioli, V. Angelopoulos, Relativistic Electrons Produced by Foreshock Disturbances Observed Upstream of Earth's Bow Shock, Physical Review Letters 117 (21) (2016) 215101. arXiv:1607.02183, doi:10.1103/PhysRevLett.117.215101.

[67] M. Oka, L. B. Wilson, III, T. D. Phan, A. J. Hull, T. Amano, M. Hoshino, M. R. Argall, O. Le Contel, O. Agapitov, D. J. Gershman, Y. V. Khotyaintsev, J. L. Burch, R. B. Torbert, C. Pollock, J. C. Dorelli, B. L. Giles, T. E. Moore, Y. Saito, L. A. Avanov, W. Paterson, R. E. Ergun, R. J. Strangeway, C. T. Russell, P. A. Lindqvist, Electron Scattering by High-frequency Whistler Waves at Earth's Bow Shock, Astrophys. J. Lett.842 (2017) L11. doi:10.3847/2041-8213/aa7759.

[68] V. Krasnoselskikh, M. Balikhin, S. N. Walker, S. Schwartz, D. Sundkvist, V. Lobzin, M. Gedalin, S. D. Bale, F. Mozer, J. Soucek, Y. Hobara, H. Comisel, The Dynamic Quasiperpendicular Shock: Cluster Discoveries, Space Sci. Rev.178 (2013) 535-598. arXiv:1303.0190, doi:10.1007/s11214-013-9972-y.

[69] S. Wing, J. R. Johnson, C. C. Chaston, M. Echim, C. P. Escoubet, B. Lavraud, C. Lemon, K. Nykyri, A. Otto, J. Raeder, C.-P. Wang, Review of Solar Wind Entry into and Transport Within the Plasma Sheet, Space Sci. Rev.184 (2014) 33-86. doi:10.1007/s11214-014-0108-9.

[70] W. Baumjohann, R. A. Treumann, Basic space plasma physics, London: Imperial College Press, 1996.

[71] E. V. Panov, J. Büchner, M. Fränz, A. Korth, S. P. Savin, K.-H. Fornaçon, I. Dandouras, H. Rème, CLUSTER observation of collisionless transport at the magnetopause, Geophys. Res. Lett.33 (2006) 15109. doi:10.1029/2006GL026556.

[72] Y. Yao, C. C. Chaston, K.-H. Glassmeier, V. Angelopoulos, Electromagnetic waves on ion gyro-radii scales across the magnetopause, Geophys. Res. Lett.38 (2011) L09102. doi:10.1029/2011GL047328.

[73] C. C. Chaston, Y. Yao, N. Lin, C. Salem, G. Ueno, Ion heating by broadband electromagnetic waves in the magnetosheath and across the magnetopause, J. Geophys. Res.118 (2013) 5579-5591. doi:10.1002/jgra.50506.

[74] J. R. Johnson, C. Z. Cheng, Global structure of mirror modes in the magnetosheath, J. Geophys. Res.102 (1997) $7179-7190$. doi:10.1029/96JA03949.

[75] C. C. Chaston, C. Salem, J. W. Bonnell, C. W. Carlson, R. E. Ergun, R. J. Strangeway, J. P. McFadden, The Turbulent Alfvénic Aurora, Physical Review Letters 100 (17) (2008) 175003. doi:10.1103/PhysRevLett.100.175003.

[76] D. N. Baker, T. I. Pulkkinen, V. Angelopoulos, W. Baumjohann, R. L. McPherron, Neutral line model of substorms: Past results and present view, J. Geophys. Res.101 (1996) 12975-13010. doi:10.1029/95JA03753.

[77] V. Angelopoulos, J. P. McFadden, D. Larson, C. W. Carlson, S. B. Mende, H. Frey, T. Phan, D. G. Sibeck, K. Glassmeier, U. Auster, E. Donovan, I. R. Mann, I. J. Rae, C. T. Russell, A. Runov, X. Zhou, L. Kepko, Tail Reconnection Triggering Substorm Onset, Science 321 (2008) 931-935. doi:10.1126/science.1160495.

[78] G. Paschmann, M. Oieroset, T. Phan, In-Situ Observations of Reconnection in Space, Space Sci. Rev.178 (2013) $385-417$. doi:10.1007/s11214-012-9957-2

[79] M. Fujimoto, I. Shinohara, H. Kojima, Reconnection and Waves: A Review with a Perspective, Space Sci. Rev.160 (2011) $123-143$. doi:10.1007/s11214-011-9807-7.

[80] V. Angelopoulos, A. Runov, X. Z. Zhou, D. L. Turner, S. A. Kiehas, S. S. Li, I. Shinohara, Electromagnetic Energy Conversion at Reconnection Fronts, Science 341 (2013) 1478-1482. doi:10.1126/science.1236992.

[81] R. L. Lysak, Electrodynamic coupling of the magnetosphere and ionosphere, Space Sci. Rev.52 (1990) 33-87. doi:10.1007/BF00704239.

[82] J. Birn, A. V. Artemyev, D. N. Baker, M. Echim, M. Hoshino, L. M. Zelenyi, Particle acceleration in the magnetotail and aurora, Space Sci. Rev.173 (2012) 49102. doi:10.1007/s11214-012-9874-4.

[83] M. G. Kivelson, C. T. Russell, Introduction to Space Physics, 1995.

[84] V. K. Jordanova, Sources, Transport, and Losses of Energetic Particles During Geomagnetic Storms, in: T. I. Pulkkinen, N. A. Tsyganenko, R. H. W. Friedel (Eds.), The Inner Magnetosphere: Physics and Modeling, Vol. 155 of Washington DC American Geophysical Union Geophysical Monograph Series, 2005, p. 9.

[85] C. F. Kennel, H. E. Petschek, Limit on Stably Trapped Particle Fluxes, J. Geophys. Res.71 (1966) 1-28.

[86] R. B. Horne, R. M. Thorne, Y. Y. Shprits, N. P. Meredith, S. A. Glauert, A. J. Smith, S. G. Kanekal, D. N. Baker, M. J. Engebretson, J. L. Posch, M. Spasojevic, U. S. Inan, J. S. Pickett, P. M. E. Decreau, Wave acceleration of electrons in the Van Allen radiation belts, Nature 437 (2005) 227-230. doi:10.1038/nature03939.

[87] R. M. Thorne, W. Li, B. Ni, Q. Ma, J. Bortnik, L. Chen, D. N. Baker, H. E. Spence, G. D. Reeves, M. G. Henderson, C. A. Kletzing, W. S. Kurth, G. B. Hospodarsky, J. B. Blake, J. F. Fennell, S. G. Claudepierre, S. G. Kanekal, Rapid local acceleration of relativistic radiation-belt electrons by magnetospheric chorus, Nature504 (2013) 411-414. doi:10.1038/nature12889.

[88] J. M. Albert, X. Tao, J. Bortnik, Aspects of Nonlinear Wave-Particle Interactions, in: D. Summers, I. U. Mann, D. N. Baker, M. Schulz (Eds.), Dynamics of the Earth's Radiation Belts and Inner Magnetosphere, American Geophysical Union, 2013. doi:10.1029/2012GM001324.

[89] J. R. Wygant, A. Keiling, C. A. Cattell, R. L. Lysak, M. Temerin, F. S. Mozer, C. A. Kletzing, J. D. Scudder, V. Streltsov, W. Lotko, C. T. Russell, Evidence for kinetic Alfvén waves and parallel electron energization at 4-6 $\mathrm{R}_{E}$ altitudes in the plasma sheet boundary layer, J. Geophys. Res.107 (2002) 1201. doi:10.1029/2001JA900113.

[90] C. E. J. Watt, R. Rankin, Electron Trapping in Shear Alfvén Waves that Power the Aurora, Physical Review Letters 102 (4) (2009) 045002. doi:10.1103/PhysRevLett.102.045002.

[91] P. A. Damiano, J. R. Johnson, C. C. Chaston, Ion temperature effects on magnetotail Alfvén wave propagation and electron energization, J. 
Geophys. Res.120 (2015) 5623-5632. doi:10.1002/2015JA021074.

[92] A. V. Artemyev, R. Rankin, M. Blanco, Electron trapping and acceleration by kinetic Alfven waves in the inner magnetosphere, J. Geophys. Res.120 (2015) 10. doi:10.1002/2015JA021781.

[93] M. Starodubtsev, C. Krafft, Resonant Cyclotron Emission of Whistler Waves by a Modulated Electron Beam, Physical Review Letters 83 (1999) 1335-1338. doi:10.1103/PhysRevLett.83.1335.

[94] B. Van Compernolle, X. An, J. Bortnik, R. M. Thorne, P. Pribyl, W. Gekelman, Excitation of Chirping Whistler Waves in a Laboratory Plasma, Physical Review Letters 114 (24) (2015) 245002. doi:10.1103/PhysRevLett.114.245002.

[95] E. M. Tejero, C. Crabtree, D. D. Blackwell, W. E. Amatucci, M. Mithaiwala, G. Ganguli, L. Rudakov, Laboratory studies of nonlinear whistler wave processes in the Van Allen radiation belts, Physics of Plasmas 22 (9) (2015) 091503. doi:10.1063/1.4928944.

[96] M. E. Viktorov, S. V. Golubev, D. A. Mansfeld, A. V. Vodopyanov, Excitation of electromagnetic waves in dense plasma during the injection of supersonic plasma flows into magnetic arch, AIP Conference Proceedings 1771 (1) (2016) 070010. doi:10.1063/1.4964234.

[97] D. Bénisti, O. Morice, L. Gremillet, A. Friou, E. Lefebvre, Nonlinear kinetic modeling of stimulated Raman scattering in a multidimensional geometrya), Physics of Plasmas 19 (5) (2012) 056301. doi:10.1063/1.3693123.

[98] E. L. Dewald, F. Hartemann, P. Michel, J. Milovich, M. Hohenberger, A. Pak, O. L. Landen, L. Divol, H. F. Robey, O. A. Hurricane, T. Döppner, F. Albert, B. Bachmann, N. B. Meezan, A. J. MacKinnon, D. Callahan, M. J. Edwards, Generation and Beaming of Early Hot Electrons onto the Capsule in Laser-Driven Ignition Hohlraums, Physical Review Letters 116 (7) (2016) 075003. doi:10.1103/PhysRevLett.116.075003.

[99] N. M. Kroll, P. L. Morton, M. N. Rosenbluth, Free-electron lasers with variable parameter wigglers, IEEE Journal of Quantum Electronics 17 (1981) 1436-1468. doi:10.1109/JQE.1981.1071285.

[100] E. D. Courant, C. Pellegrini, W. Zakowicz, High-energy inverse free-electron-laser accelerator, Phys. Rev. A32 (1985) $2813-2823$. doi:10.1103/PhysRevA.32.2813.

[101] R. W. Clark, D. G. Swanson, P. Korn, F. Sandel, S. Robertson, C. B. Wharton, High power fundamental and harmonic resonant ion cyclotron heating in a mirror machine, Physics of Fluids 17 (1974) 1322-1328. doi:10.1063/1.1694884.

[102] H. Jhang, S. G. Lee, S. S. Kim, B. H. Park, J. G. Bak, Stabilization of Interchange Modes in Mirror Plasmas by a Nonlinear rf-Plasma Wave Coupling Process, Physical Review Letters 95 (3) (2005) 035005. doi:10.1103/PhysRevLett.95.035005.

[103] T. Nakamura, K. Mima, H. Sakagami, T. Johzaki, Electron surface acceleration on a solid capillary target inner wall irradiated with ultraintense laser pulses, Physics of Plasmas 14 (5) (2007) 053112. doi:10.1063/1.2731383.

[104] V. I. Arnold, V. V. Kozlov, A. I. Neishtadt, Mathematical Aspects of Classical and Celestial Mechanics, 3rd Edition, Dynamical Systems III. Encyclopedia of Mathematical Sciences, Springer-Verlag, New York, 2006.

[105] A. Neishtadt, Passage through a separatrix in a resonance problem with a slowly-varying parameter, Journal of Applied Mathematics and Mechanics 39 (1975) 594-605. doi:10.1016/0021-8928(75)90060-X.

[106] A. P. Itin, A. I. Neishtadt, A. A. Vasiliev, Captures into resonance and scattering on resonance in dynamics of a charged relativistic particle in magnetic field and electrostatic wave, Physica D: Nonlinear Phenomena 141 (2000) 281-296. doi:10.1016/S0167-2789(00)00039-7.

[107] D. Dolgopyat, Repulsion from Resonances, in: Memoires De La Societe Mathematique De France, Vol. 128 of Memoires De La Societe Mathematique De France, 2012.

[108] J. M. Albert, Diffusion by one wave and by many waves, J. Geophys. Res.115 (2010) 0. doi:10.1029/2009JA014732.

[109] A. I. Neishtadt, A. V. Artemyev, L. M. Zelenyi, D. L. Vainshtein, Surfatron acceleration in electromagnetic waves with a low phase velocity, JETP Letters 89 (2009) 441-447. doi:10.1134/S0021364009090045.

[110] A. Neishtadt, A. Vasiliev, A. Artemyev, Resonance-induced surfatron acceleration of a relativistic particle, Moscow Mathematical Journal 11 (3) (2011) 531-545.

[111] A. I. Neishtadt, On Adiabatic Invariance in Two-Frequency Systems, in Hamiltonian Systems with Three or More Degrees of Freedom, ed. Sim C., NATO ASI Series C. Dordrecht: Kluwer Acad. Publ. 533 (1999) 193-213. doi:10.1063/1.166236.

[112] T. F. Bell, The nonlinear gyroresonance interaction between energetic electrons and coherent VLF waves propagating at an arbitrary angle with respect to the earth's magnetic field, J. Geophys. Res.89 (1984) 905-918. doi:10.1029/JA089iA02p00905.

[113] M. A. Malkov, G. M. Zaslavskii, Wave-particle resonant interaction in a weak magnetic field, Physics Letters A 106 (1984) $257-260$. doi:10.1016/0375-9601(84)91022-3.

[114] F. Valentini, P. Veltri, A. Mangeney, Magnetic-field effects on nonlinear electrostatic-wave Landau damping, Phys. Rev. E71 (1) (2005) 016402. doi:10.1103/PhysRevE.71.016402.

[115] D. Vainchtein, I. Mezić, Capture into Resonance: A Method for Efficient Control, Physical Review Letters 93 (8) (2004) 084301. doi:10.1103/PhysRevLett.93.084301.

[116] A. I. Neishtadt, A. V. Artemyev, L. M. Zelenyi, Regular and chaotic charged particle dynamics in low frequency waves and role of separatrix crossings, Regular and Chaotic Dynamics 15 (2010) 564-574. doi:10.1134/S1560354710040118.

[117] C. F. F. Karney, Stochastic ion heating by a lower hybrid wave, Physics of Fluids 21 (1978) 1584-1599. doi:10.1063/1.862406.

[118] C. F. F. Karney, Stochastic ion heating by a lower hybrid wave. II, Physics of Fluids 22 (1979) 2188-2209. arXiv:arXiv:physics/0501034, doi: $10.1063 / 1.862512$.

[119] S. Takeuchi, K. Sakai, M. Matsumoto, R. Sugihara, Unlimited acceleration of a charged particle by an electromagnetic wave with a purely transverse electric field, Physics Letters A 122 (1987) 257-261. doi:10.1016/0375-9601(87)90818-8.

[120] S. Takeuchi, New particle accelerations by magnetized plasma shock waves, Physics of Plasmas 12 (10) (2005) 102901. doi: $10.1063 / 1.2080520$

[121] A. Runov, V. Angelopoulos, V. A. Sergeev, K. Glassmeier, U. Auster, J. McFadden, D. Larson, I. Mann, Global properties of magnetotail current sheet flapping: THEMIS perspectives, Annales Geophysicae 27 (2009) 319-328.

[122] M. I. Sitnov, M. Swisdak, A. V. Divin, Dipolarization fronts as a signature of transient reconnection in the magnetotail, J. Geophys. Res.114 (2009) A04202. doi:10.1029/2008JA013980.

[123] A. Runov, V. Angelopoulos, M. I. Sitnov, V. A. Sergeev, J. Bonnell, J. P. McFadden, D. Larson, K. Glassmeier, U. Auster, THEMIS observations of an earthward-propagating dipolarization front, Geophys. Res. Lett.36 (2009) L14106. doi:10.1029/2009GL038980. 
[124] T. Sundberg, J. A. Slavin, S. A. Boardsen, B. J. Anderson, H. Korth, G. C. Ho, D. Schriver, V. M. Uritsky, T. H. Zurbuchen, J. M. Raines, D. N. Baker, S. M. Krimigis, R. L. McNutt, Jr., S. C. Solomon, MESSENGER observations of dipolarization events in Mercury's magnetotail, J. Geophys. Res.117 (2012) 0. doi:10.1029/2012JA017756.

[125] S. Kasahara, E. A. Kronberg, T. Kimura, C. Tao, S. V. Badman, A. Masters, A. Retinò, N. Krupp, M. Fujimoto, Asymmetric distribution of reconnection jet fronts in the Jovian nightside magnetosphere, J. Geophys. Res.118 (2013) 375-384. doi:10.1029/2012JA018130.

[126] A. V. Artemyev, G. Zimbardo, A. Y. Ukhorskiy, M. Fujimoto, Preferential acceleration of heavy ions in the reconnection outflow region. Drift and surfatron ion acceleration, Astron. Astrophys.562 (2014) A58. doi:10.1051/0004-6361/201322462.

[127] A. V. Artemyev, V. N. Lutsenko, A. A. Petrukovich, Ion resonance acceleration by dipolarization fronts: analytic theory and spacecraft observation., Annales Geophysicae 30 (2012) 317324.

[128] A. Y. Ukhorskiy, M. I. Sitnov, V. G. Merkin, A. V. Artemyev, Rapid acceleration of protons upstream of earthward propagating dipolarization fronts, J. Geophys. Res.118 (2013) 4952-4962. doi:10.1002/jgra.50452.

[129] A. V. Artemyev, S. Kasahara, , A. Y. Ukhorskiy, M. Fujimoto, Acceleration of ions in the Jupiter magnetotail: role of resonant interaction with dipolarization fronts., Planatary Space Science82-83 (2013) 134-148. doi:10.1016/j.pss.2013.04.013.

[130] A. V. Artemyev, A. A. Vasiliev, Resonant ion acceleration by plasma jets: Effects of jet breaking and the magnetic-field curvature, Phys. Rev. E91 (5) (2015) 053104. doi:10.1103/PhysRevE.91.053104.

[131] A. V. Artemyev, A. I. Neishtadt, L. M. Zelenyi, D. L. Vainchtein, Adiabatic description of capture into resonance and surfatron acceleration of charged particles by electromagnetic waves, Chaos 20 (4) (2010) 043128. doi:10.1063/1.3518360.

[132] X. Zhou, V. Angelopoulos, V. A. Sergeev, A. Runov, Accelerated ions ahead of earthward propagating dipolarization fronts, J. Geophys. Res.115 (2010) A00I03. doi:10.1029/2010JA015481.

[133] F. S. Mozer, O. Agapitov, V. Krasnoselskikh, S. Lejosne, G. D. Reeves, I. Roth, Direct Observation of Radiation-Belt Electron Acceleration from Electron-Volt Energies to Megavolts by Nonlinear Whistlers, Physical Review Letters 113 (3) (2014) 035001. doi:10.1103/PhysRevLett.113.035001.

[134] A. V. Artemyev, O. Agapitov, F. Mozer, V. Krasnoselskikh, Thermal electron acceleration by localized bursts of electric field in the radiation belts, Geophys. Res. Lett.41 (2014) 57345739. doi:10.1002/2014GL061248.

[135] F. S. Mozer, O. Agapitov, A. Artemyev, J. F. Drake, V. Krasnoselskikh, S. Lejosne, I. Vasko, Time domain structures: What and where they are, what they do, and how they are made, Geophys. Res. Lett.42 (2015) 36273638. doi:10.1002/2015GL063946.

[136] I. Y. Vasko, O. V. Agapitov, F. S. Mozer, A. V. Artemyev, Thermal electron acceleration by electric field spikes in the outer radiation belt: Generation of field-aligned pitch angle distributions, J. Geophys. Res.120 (2015) 8616-8632. doi:10.1002/2015JA021644.

[137] A. Vasiliev, A. Neishtadt, A. Artemyev, Nonlinear dynamics of charged particles in an oblique electromagnetic wave, Physics Letters A 375 (2011) 3075-3079. doi:10.1016/j.physleta.2011.06.055.

[138] A. I. Neishtadt, B. A. Petrovichev, A. A. Chernikov, Particle entrainment into unlimited acceleration, Soviet Journal of Plasma Physics 15 (1989) 1021-1023.

[139] A. A. Chernikov, G. Schmidt, A. I. Neishtadt, Unlimited particle acceleration by waves in a magnetic field, Physical Review Letters 68 (1992) 1507-1510. doi:10.1103/PhysRevLett.68.1507.

[140] A. P. Itin, Trapping and scattering of a relativistic charged particle by resonance in a magnetic field and an electromagnetic wave, Plasma Physics Reports 28 (2002) 592-603. doi:10.1134/1.1494058.

[141] D. L. Vainchtein, E. V. Rovinsky, L. M. Zelenyi, A. I. Neishtadt, Resonances and Particle Stochastization in Nonhomogeneous Electromagnetic Fields, Journal of NonLinear Science 14 (2004) 173-205. doi:10.1007/s00332-003-0576-7.

[142] S. V. Bulanov, A. S. Sakharov, Acceleration of particles captured by a strong potential wave with a curved wave front in a magnetic field, Soviet Journal of Experimental and Theoretical Physics Letters 44 (1986) 543-546.

[143] S. V. Bulanov, V. Krasnoselskikh, Particle Acceleration in Expanding and Moving Shocks, in: A. Wilson, et al. (Eds.), Magnetic Fields and Solar Processes, Vol. 448 of ESA Special Publication, 1999, p. 1047.

[144] N. S. Erokhin, S. S. Moiseev, R. Z. Sagdeev, Relativistic Surfing in Inhomogeneous Plasma and the Origin of Energetic Cosmic-Rays, Soviet Astronomy Letters 15 (1989) 1.

[145] A. N. Erokhin, N. S. Erokhin, V. P. Milant'ev, Trapping of high-energy electrons into regime of surfatron acceleration by electromagnetic waves in space plasma, Plasma Physics Reports 38 (2012) 396-406. doi:10.1134/S1063780X12040022.

[146] A. Artemyev, V. Krasnoselskikh, O. Agapitov, D. Mourenas, G. Rolland, Non-diffusive resonant acceleration of electrons in the radiation belts., Physics of Plasmas 19 (2012) 122901. doi:10.1063/1.4769726.

[147] A. Osmane, A. M. Hamza, Relativistic surfatron process for Landau resonant electrons in radiation belts, Nonlinear Processes in Geophysics 21 (2014) 115-125. arXiv:1402.1691, doi:10.5194/npg-21-115-2014.

[148] A. V. Artemyev, A. A. Vasiliev, D. Mourenas, O. Agapitov, V. Krasnoselskikh, Nonlinear electron acceleration by oblique whistler waves: Landau resonance vs. cyclotron resonance., Physics of Plasmas 20 (2013) 122901. doi:10.1063/1.4836595.

[149] D. L. Vainchtein, A. A. Vasiliev, A. I. Neishtadt, Electron dynamics in a parabolic magnetic field in the presence of an electrostatic wave, Plasma Physics Reports 35 (2009) 1021-1031. doi:10.1134/S1063780X09120046.

[150] A. Neishtadt, D. Vainchtein, A. Vasiliev, Dynamics of electrons in a parabolic magnetic field perturbed by an electromagnetic wave, Plasma Physics and Controlled Fusion 53 (8) (2011) 085014. doi:10.1088/0741-3335/53/8/085014.

[151] A. Artemyev, O. Agapitov, D. Mourenas, V. Krasnoselskikh, V. Shastun, F. Mozer, Oblique whistler-mode waves in the earth's inner magnetosphere: Energy distribution, origins, and role in radiation belt dynamics, Space Science Reviews 200 (2016) 261-355. doi:10.1007/s11214016-0252-5.

URL http: //dx.doi.org/10.1007/s11214-016-0252-5

[152] Y. Omura, N. Furuya, D. Summers, Relativistic turning acceleration of resonant electrons by coherent whistler mode waves in a dipole magnetic field, J. Geophys. Res.112 (2007) 6236. doi:10.1029/2006JA012243.

[153] D. Summers, Y. Omura, Ultra-relativistic acceleration of electrons in planetary magnetospheres, Geophys. Res. Lett.34 (2007) 24205. doi:10.1029/2007GL032226.

[154] A. V. Artemyev, A. A. Vasiliev, D. Mourenas, A. I. Neishtadt, O. V. Agapitov, V. Krasnoselskikh, Probability of relativistic electron trapping 
by parallel and oblique whistler-mode waves in Earth's radiation belts, Physics of Plasmas 22 (11) (2015) 112903. doi:10.1063/1.4935842.

[155] G. M. Zaslavskii, N. N. Filonenko, Stochastic Instability of Trapped Particles and Conditions of Applicability of the Quasi-linear Approximation, Soviet Journal of Experimental and Theoretical Physics 27 (1968) 851.

[156] A. Zaslavsky, C. Krafft, L. Gorbunov, A. Volokitin, Wave-particle interaction at double resonance, Phys. Rev. E77 (5) (2008) 056407. doi:10.1103/PhysRevE.77.056407.

[157] D. R. Shklyar, G. Zimbardo, Particle dynamics in the field of two waves in a magnetoplasma, Plasma Physics and Controlled Fusion 56 (9) (2014) 095002. doi:10.1088/0741-3335/56/9/095002.

[158] A. Artemyev, D. Vainchtein, A. Neishtadt, L. Zelenyi, Resonant acceleration of charged particles in the presence of random fluctuations, Phys. Rev. E84 (4) (2011) 046213. doi:10.1103/PhysRevE.84.046213.

[159] A. V. Artemyev, D. Mourenas, O. V. Agapitov, D. L. Vainchtein, F. S. Mozer, V. V. Krasnoselskikh, Stability of relativistic electron trapping by strong whistler or electromagnetic ion cyclotron waves, Physics of Plasmas 22 (2015) 082901. doi:10.1063/1.4927774.

[160] J. M. Albert, Gyroresonant interactions of radiation belt particles with a monochromatic electromagnetic wave, J. Geophys. Res.105 (2000) 21191. doi:10.1029/2000JA000008.

[161] A. Y. Ukhorskiy, M. I. Sitnov, Dynamics of Radiation Belt Particles, Space Sci. Rev.179 (2013) 545-578. doi:10.1007/s11214-012-9938-5.

[162] L. D. Landau, E. M. Lifshitz, Vol. 1: Mechanics, Course of Theoretical Physics, Oxford: Pergamon, 1988.

[163] A. Artemyev, D. Vainchtein, A. Neishtadt, L. Zelenyi, Stability of relativistic surfatron acceleration, Phys. Rev. E89 (4) (2014) 043106. doi:10.1103/PhysRevE.89.043106.

[164] I. B. Bernstein, J. M. Greene, M. D. Kruskal, Exact Nonlinear Plasma Oscillations, Physical Review 108 (1957) 546-550. doi:10.1103/PhysRev.108.546

[165] V. I. Karpman, F. F. Cap, Non-linear waves in dispersive media., 1975

[166] A. A. Galeev, R. Z. Sagdeev, Nonlinear Plasma Theory, in: A. M. A. Leontovich (Ed.), Reviews of Plasma Physics, Volume 7, Vol. 7 of Reviews of Plasma Physics, 1979, p. 1.

[167] H. Matsumoto, H. Kojima, T. Miyatake, Y. Omura, M. Okada, I. Nagano, M. Tsutsui, Electrotastic Solitary Waves (ESW) in the magnetotail: BEN wave forms observed by GEOTAIL, Geophys. Res. Lett.21 (1994) 2915-2918. doi:10.1029/94GL01284.

[168] R. E. Ergun, C. W. Carlson, J. P. McFadden, F. S. Mozer, G. T. Delory, W. Peria, C. C. Chaston, M. Temerin, I. Roth, L. Muschietti, R. Elphic, R. Strangeway, R. Pfaff, C. A. Cattell, D. Klumpar, E. Shelley, W. Peterson, E. Moebius, L. Kistler, FAST satellite observations of large-amplitude solitary structures, Geophys. Res. Lett.25 (1998) 2041-2044. doi:10.1029/98GL00636.

[169] C. Cattell, J. R. Wygant, K. Goetz, K. Kersten, P. J. Kellogg, T. von Rosenvinge, S. D. Bale, I. Roth, M. Temerin, M. K. Hudson, R. A. Mewaldt, M. Wiedenbeck, M. Maksimovic, R. Ergun, M. Acuna, C. T. Russell, Discovery of very large amplitude whistler-mode waves in Earth's radiation belts, Geophys. Res. Lett.35 (2008) 1105. doi:10.1029/2007GL032009.

[170] C. M. Cully, J. W. Bonnell, R. E. Ergun, THEMIS observations of long-lived regions of large-amplitude whistler waves in the inner magnetosphere, Geophys. Res. Lett.35 (2008) 17. doi:10.1029/2008GL033643.

[171] M. A. Balikhin, Y. Y. Shprits, S. N. Walker, L. Chen, N. Cornilleau-Wehrlin, I. Dandouras, O. Santolik, C. Carr, K. H. Yearby, B. Weiss, Observations of discrete harmonics emerging from equatorial noise, Nature Communications 6 (2015) 7703. doi:10.1038/ncomms8703.

[172] F. D. Wilder, R. E. Ergun, K. A. Goodrich, M. V. Goldman, D. L. Newman, D. M. Malaspina, A. N. Jaynes, S. J. Schwartz, K. J. Trattner, J. L. Burch, M. R. Argall, R. B. Torbert, P.-A. Lindqvist, G. Marklund, O. Le Contel, L. Mirioni, Y. V. Khotyaintsev, R. J. Strangeway, C. T. Russell, C. J. Pollock, B. L. Giles, F. Plaschke, W. Magnes, S. Eriksson, J. E. Stawarz, A. P. Sturner, J. C. Holmes, Observations of whistler mode waves with nonlinear parallel electric fields near the dayside magnetic reconnection separatrix by the Magnetospheric Multiscale mission, Geophys. Res. Lett.43 (2016) 5909-5917. doi:10.1002/2016GL069473.

[173] F. S. Mozer, O. A. Agapitov, A. Artemyev, J. L. Burch, R. E. Ergun, B. L. Giles, D. Mourenas, R. B. Torbert, T. D. Phan, I. Vasko, Magnetospheric Multiscale Satellite Observations of Parallel Electron Acceleration in Magnetic Field Reconnection by Fermi Reflection from Time Domain Structures, Physical Review Letters 116 (14) (2016) 145101. doi:10.1103/PhysRevLett.116.145101.

[174] R. M. Thorne, B. Ni, X. Tao, R. B. Horne, N. P. Meredith, Scattering by chorus waves as the dominant cause of diffuse auroral precipitation, Nature467 (2010) 943-946. doi:10.1038/nature09467.

[175] O. V. Agapitov, A. V. Artemyev, D. Mourenas, F. S. Mozer, V. Krasnoselskikh, Nonlinear local parallel acceleration of electrons through Landau trapping by oblique whistler mode waves in the outer radiation belt, Geophys. Res. Lett.42 (2015) 10. doi:10.1002/2015GL066887.

[176] J. C. Foster, P. J. Erickson, Y. Omura, D. N. Baker, C. A. Kletzing, S. G. Claudepierre, Van allen probes observations of prompt mev radiation belt electron acceleration in nonlinear interactions with vlf chorus, Journal of Geophysical Research: Space Physics 122 (1) (2017) 324-339. doi:10.1002/2016JA023429. URL http: //dx.doi.org/10.1002/2016JA023429

[177] N. G. Van Kampen, Stochastic Processes in Physics and Chemistry, 3rd Edition, North Holland, 2003.

[178] J. Bortnik, R. M. Thorne, U. S. Inan, Nonlinear interaction of energetic electrons with large amplitude chorus, Geophys. Res. Lett.35 (2008) 21102. doi:10.1029/2008GL035500.

[179] F. S. Mozer, A. Artemyev, O. V. Agapitov, D. Mourenas, I. Vasko, Near-relativistic electron acceleration by Landau trapping in time domain structures, Geophys. Res. Lett.43 (2016) 508-514. doi:10.1002/2015GL067316.

[180] Y. Omura, Y. Miyashita, M. Yoshikawa, D. Summers, M. Hikishima, Y. Ebihara, Y. Kubota, Formation process of relativistic electron flux through interaction with chorus emissions in the Earth's inner magnetosphere, J. Geophys. Res.120 (2015) 9545-9562. doi:10.1002/2015JA021563.

[181] Y.-K. Hsieh, Y. Omura, Study of wave-particle interactions for whistler mode waves at oblique angles by utilizing the gyroaveraging method, Radio Science 52 (10) (2017) 1268-1281, 2017RS006245. doi:10.1002/2017RS006245. URL http://dx.doi.org/10.1002/2017RS006245

[182] A. V. Artemyev, A. I. Neishtadt, A. A. Vasiliev, D. Mourenas, Probabilistic approach to nonlinear wave-particle resonant interaction, Phys. Rev. E95 (2) (2017) 023204. doi:10.1103/PhysRevE.95.023204.

[183] A. V. Artemyev, A. I. Neishtadt, A. A. Vasiliev, D. Mourenas, Kinetic equation for systems with resonant captures and scatterings, ArXiv e-printsarXiv: 1710.04489. 
[184] X. Leoncini, A. Vasiliev, A. Artemyev, Resonance controlled transport in phase space, Physica D: Nonlinear Phenomenadoi:https://doi.org/10.1016/j.physd.2017.09.010.

URL http://www.sciencedirect.com/science/article/pii/S0167278917302877

[185] J. Li, J. Bortnik, L. Xie, Z. Pu, L. Chen, B. Ni, X. Tao, R. M. Thorne, S. Fu, Z. Yao, R. Guo, Comparison of formulas for resonant interactions between energetic electrons and oblique whistler-mode waves, Physics of Plasmas 22 (5) (2015) 052902. doi:10.1063/1.4914852. 